<smiles>[CH]=C</smiles><smiles>C1=CCCC1</smiles>

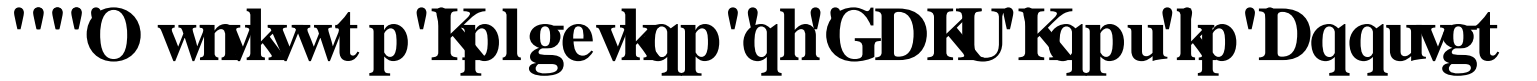

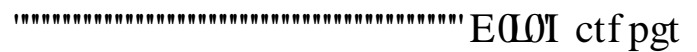

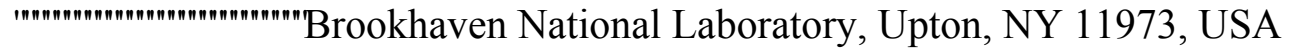

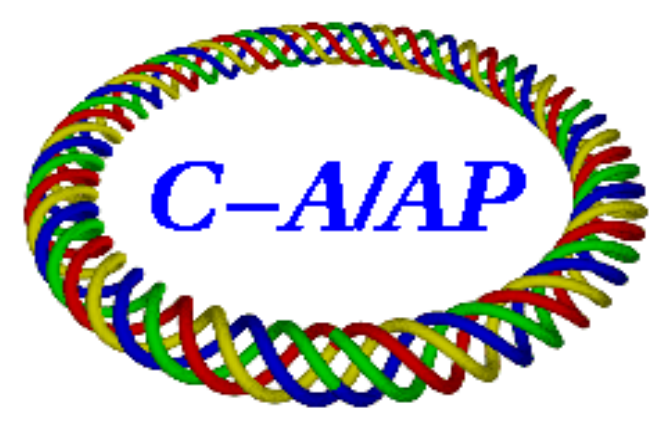

\section{Collider-Accelerator Department Brookhaven National Laboratory Upton, NY 11973}

Notice: This document has been authorized by employees of Brookhaven Science Associates, LLCunder Contract No. DE-AC02-98CH10886 with the U.S. Departm ent of En ergy. The United States Governm ent retains a nonexclusive, paid-up, irrevocable, world-wide license topublish or reproduce the published formof this document, or allow others to do so, for United States Government purposes. 


\title{
Multiturn Injection of EBIS Ions in Booster
}

\author{
C.J. Gardner
}

September 1, 2010

Ions from EBIS are injected into Booster after acceleration by an RFQ and a Linac. The velocity of the ions at Booster injection is $c \beta$ where $c$ is the velocity of light and

$$
\beta=0.0655 \text {. }
$$

The kinetic energy is

$$
W=m c^{2}(\gamma-1)
$$

where $m$ is the ion mass and

$$
\gamma=\left(1-\beta^{2}\right)^{-1 / 2}
$$

Putting in numbers one gets a kinetic energy of approximately $2 \mathrm{MeV}$ per nucleon for each ion. The revolution period at injection is $10.276 \mu \mathrm{s}$.

The ions in the EBIS trap are delivered in a beam pulse that ranges from 10 to $40 \mu \mathrm{s}$ in length. This amounts to 1 to 4 turns around the machine. The transverse emittance (un-normalized) of EBIS beams just prior to injection into Booster is $11 \pi \mathrm{mm}$ milliradians in both planes. This is an order of magnitude larger than the nominal $1 \pi \mathrm{mm}$ milliradians for Tandem beams.

Injection proceeds by means of an electrostatic inflector in the $\mathrm{C} 3$ straight section and four programmable injection dipoles in the $\mathrm{C} 1, \mathrm{C} 3, \mathrm{C} 7$, and D1 straights. These devices have been in use for many years for the injection of ions from Tandem as described in [1] and [2]. The inflector brings the incoming beam to the edge of the Booster acceptance and the dipoles produce a closed orbit bump that initially places the closed orbit near the septum at the inflector exit. During injection the orbit bump must be collapsed at a rate that keeps the injected beam from hitting the septum while continuing to allow beam to be injected into the machine acceptance. The process is discussed in [2] and [3]. There it is assumed that the 
injected beam moves with the closed orbit as the bump collapses. In the present report this is shown to be a valid approximation if the bump collapses sufficiently slowly. It is also shown that by judiciously choosing the horizontal tune and the initial distance of the closed orbit from the septum one can inject up to 4 turns of EBIS beams without loss on the septum. The reason for wanting to inject over a period of 4 rather than fewer turns is that this allows the beam to be distributed over a larger area of the Booster acceptance, thereby reducing the space charge force on the beam particles.

The framework for the injection process is set up in Sections $\mathbf{1}$ through $\mathbf{5}$.

The injection dipoles and the capabilities of their power supplies are reviewed in Section 6. The injection process is illustrated with several examples in Section 7.

\section{Injection Bump}

The injection bump is produced by four dipole magnets located in the injection region with positions

$$
s_{1}<s_{2}<s_{I}<s_{3}<s_{4}
$$

where $s_{I}$ is the point of injection. Let $\phi_{j}$ be the angular kick produced by the dipole at $s_{j}$ and let

$$
\Phi_{j}=\left(\begin{array}{c}
0 \\
\phi_{j}
\end{array}\right), \quad \mathbf{X}_{c}=\left(\begin{array}{c}
X_{c} \\
X_{c}^{\prime}
\end{array}\right), \quad \mathbf{M}_{j i}=\left(\begin{array}{cc}
a_{j i} & b_{j i} \\
c_{j i} & d_{j i}
\end{array}\right)
$$

where $X_{c}, X_{c}^{\prime}$ are the horizontal position and angle of the bump at $s_{I}$ and $\mathbf{M}_{j i}$ is the transfer matrix from $s_{i}$ to $s_{j}$. The elements of the matrix are

$$
\begin{gathered}
a_{j i}=\sqrt{\beta_{j} / \beta_{i}}\left(\cos \Delta \psi+\alpha_{i} \sin \Delta \psi\right), \quad b_{j i}=\sqrt{\beta_{j} \beta_{i}} \sin \Delta \psi \\
c_{j i}=-\left(\frac{\alpha_{j}-\alpha_{i}}{\sqrt{\beta_{j} \beta_{i}}}\right) \cos \Delta \psi-\left(\frac{1+\alpha_{j} \alpha_{i}}{\sqrt{\beta_{j} \beta_{i}}}\right) \sin \Delta \psi \\
d_{j i}=\sqrt{\beta_{i} / \beta_{j}}\left(\cos \Delta \psi-\alpha_{j} \sin \Delta \psi\right)
\end{gathered}
$$

where $\alpha_{j}$ and $\beta_{j}$ are the Courant-Snyder lattice parameters at the point $s_{j}$. The phase

$$
\Delta \psi=\psi_{j}-\psi_{i}
$$


is the betatron phase advance from $s_{i}$ to $s_{j}$. We also have

$$
d_{j i}=a_{i j} .
$$

If we require that four dipoles produce no closed orbit distortion outside the injection region then we must have

$$
\Phi_{4}+\mathbf{M}_{43} \Phi_{3}+\mathbf{M}_{42} \Phi_{2}+\mathbf{M}_{41} \Phi_{1}=\mathbf{0}
$$

and position and angle of the closed orbit at the injection point $s_{I}$ are given by

$$
\mathbf{X}_{c}=\mathbf{M}_{I 2} \Phi_{2}+\mathbf{M}_{I 1} \Phi_{1}
$$

These equations can be inverted to obtain the kicks $\phi_{j}$ in terms of $X_{c}$ and $X_{c}^{\prime}$. Thus

$$
\left(\begin{array}{c}
\phi_{1} \\
\phi_{2}
\end{array}\right)=\frac{1}{b_{21}}\left(\begin{array}{rr}
d_{I 2} & -b_{I 2} \\
-d_{I 1} & b_{I 1}
\end{array}\right)\left(\begin{array}{c}
X_{c} \\
X_{c}^{\prime}
\end{array}\right)
$$

and

$$
\left(\begin{array}{l}
\phi_{3} \\
\phi_{4}
\end{array}\right)=\frac{1}{b_{43}}\left(\begin{array}{rr}
-b_{41} & -b_{42} \\
b_{31} & b_{32}
\end{array}\right)\left(\begin{array}{l}
\phi_{1} \\
\phi_{2}
\end{array}\right) .
$$

\section{Turn-by-Turn Evolution of Particle Position and Angle}

The turn-by-turn evolution of the position and angle of a particle launched from the injection point $s_{I}$ is determined by the four kicks $\phi_{j}$ and the one-turn matrix

$$
\mathbf{M}=\mathbf{M}_{I 1} \mathbf{N M}_{4 I}
$$

Here $\mathbf{N}$ is the transfer matrix from $s_{4}$ around the machine to $s_{1}$. Writing

$$
\mathbf{M}=\left(\begin{array}{ll}
M_{11} & M_{12} \\
M_{21} & M_{22}
\end{array}\right)
$$

we have

$$
\begin{array}{r}
M_{11}=\cos \left(2 \pi Q_{H}\right)+\alpha_{I} \sin \left(2 \pi Q_{H}\right), \quad M_{12}=\beta_{I} \sin \left(2 \pi Q_{H}\right) \\
M_{21}=-\gamma_{I} \sin \left(2 \pi Q_{H}\right), \quad M_{22}=\cos \left(2 \pi Q_{H}\right)-\alpha_{I} \sin \left(2 \pi Q_{H}\right)
\end{array}
$$

where $\alpha_{I}, \beta_{I}$, and $\gamma_{I}$ are the Courant-Snyner lattice parameters at $s_{I}$ and $Q_{H}$ is the horizontal tune. 
Let

$$
\mathbf{X}=\left(\begin{array}{c}
X \\
X^{\prime}
\end{array}\right), \quad \mathbf{Y}=\left(\begin{array}{c}
Y \\
Y^{\prime}
\end{array}\right)
$$

where $X$ and $X^{\prime}$ are the initial position and angle at $s_{I}$, and $Y$ and $Y^{\prime}$ are the position and angle after one turn. We then have

$$
\begin{gathered}
\mathbf{X}_{3}=\mathbf{M}_{3 I} \mathbf{X}+\Phi_{3} \\
\mathbf{X}_{4}=\mathbf{M}_{43} \mathbf{X}_{3}+\Phi_{4} \\
\mathbf{X}_{1}=\mathbf{N X}_{4}+\Phi_{1} \\
\mathbf{X}_{2}=\mathbf{M}_{21} \mathbf{X}_{1}+\Phi_{2}
\end{gathered}
$$

at $s_{3}, s_{4}, s_{1}$, and $s_{2}$, respectively, and

$$
\mathbf{Y}=\mathbf{M}_{I 2} \mathbf{X}_{2}
$$

Thus

$$
\begin{gathered}
\mathbf{Y}=\mathbf{M}_{I 1} \mathbf{X}_{1}+\mathbf{M}_{I 2} \Phi_{2} \\
\mathbf{Y}=\mathbf{M}_{I 1} \mathbf{N X}_{4}+\mathbf{M}_{I 1} \Phi_{1}+\mathbf{M}_{I 2} \Phi_{2} \\
\mathbf{X}_{4}=\mathbf{M}_{4 I} \mathbf{X}+\mathbf{M}_{43} \Phi_{3}+\Phi_{4} \\
\mathbf{Y}=\mathbf{M X}+\mathbf{M}_{I 1} \mathbf{N}\left(\mathbf{M}_{43} \Phi_{3}+\Phi_{4}\right)+\mathbf{M}_{I 1} \Phi_{1}+\mathbf{M}_{I 2} \Phi_{2} \\
\mathbf{Y}=\mathbf{M X}+\mathbf{M}\left(\mathbf{M}_{3 I}^{-1} \Phi_{3}+\mathbf{M}_{4 I}^{-1} \Phi_{4}\right)+\mathbf{M}_{I 1} \Phi_{1}+\mathbf{M}_{I 2} \Phi_{2}
\end{gathered}
$$

and we can write

$$
\mathbf{Y}-\mathbf{X}_{b}=\mathbf{M}\left(\mathbf{X}-\mathbf{X}_{a}\right)
$$

where

$$
\mathbf{X}_{a}=-\left(\mathbf{M}_{3 I}^{-1} \Phi_{3}+\mathbf{M}_{4 I}^{-1} \Phi_{4}\right)
$$

and

$$
\mathbf{X}_{b}=\mathbf{M}_{I 1} \Phi_{1}+\mathbf{M}_{I 2} \Phi_{2}
$$

Writing

$$
\mathbf{X}_{a}=\left(\begin{array}{c}
X_{a} \\
X_{a}^{\prime}
\end{array}\right), \quad \mathbf{X}_{b}=\left(\begin{array}{c}
X_{b} \\
X_{b}^{\prime}
\end{array}\right)
$$

we then have

$$
\begin{gathered}
X_{a}=b_{3 I} \phi_{3}+b_{4 I} \phi_{4} \\
X_{a}^{\prime}=-a_{3 I} \phi_{3}-a_{4 I} \phi_{4}
\end{gathered}
$$




$$
\begin{aligned}
& X_{b}=b_{I 1} \phi_{1}+b_{I 2} \phi_{2} \\
& X_{b}^{\prime}=d_{I 1} \phi_{1}+d_{I 2} \phi_{2}
\end{aligned}
$$

and

$$
\begin{gathered}
Y=X_{b}+M_{11}\left(X-X_{a}\right)+M_{12}\left(X^{\prime}-X_{a}^{\prime}\right) \\
Y^{\prime}=X_{b}^{\prime}+M_{21}\left(X-X_{a}\right)+M_{22}\left(X^{\prime}-X_{a}^{\prime}\right) .
\end{gathered}
$$

\section{Time Dependent Injection Bump}

Let us specify bump positions

$$
X_{c n}=X_{c}\left(T_{n}\right)
$$

and angles

$$
X_{c n}^{\prime}=X_{c}^{\prime}\left(T_{n}\right)
$$

at times $T_{n}$ where

$$
T_{0}<T_{1}<T_{2}<T_{3}<\cdots<T_{N} .
$$

Then the four kicks at these times are given by

$$
\left(\begin{array}{c}
\phi_{1 n} \\
\phi_{2 n}
\end{array}\right)=\frac{1}{b_{21}}\left(\begin{array}{rr}
d_{I 2} & -b_{I 2} \\
-d_{I 1} & b_{I 1}
\end{array}\right)\left(\begin{array}{l}
X_{c n} \\
X_{c n}^{\prime}
\end{array}\right)
$$

and

$$
\left(\begin{array}{l}
\phi_{3 n} \\
\phi_{4 n}
\end{array}\right)=\frac{1}{b_{43}}\left(\begin{array}{rr}
-b_{41} & -b_{42} \\
b_{31} & b_{32}
\end{array}\right)\left(\begin{array}{l}
\phi_{1 n} \\
\phi_{2 n}
\end{array}\right) .
$$

For times $t$ in the range

$$
T_{n} \leq t<T_{n+1}
$$

we take

$$
\phi_{j}(t)=\phi_{j n}+m_{j n}\left(t-T_{n}\right)
$$

where

$$
\begin{array}{ll}
m_{j 0}=\frac{\phi_{j 1}-\phi_{j 0}}{T_{1}-T_{0}}, & m_{j 1}=\frac{\phi_{j 2}-\phi_{j 1}}{T_{2}-T_{1}} \\
m_{j 2}=\frac{\phi_{j 3}-\phi_{j 2}}{T_{3}-T_{2}}, & m_{j 3}=\frac{\phi_{j 4}-\phi_{j 3}}{T_{4}-T_{3}}
\end{array}
$$


and so on. For

$$
t \geq T_{N}
$$

we take

$$
\phi_{j}(t)=\phi_{j N}
$$

We shall take the unit of time to be one revolution period. A particle launched from the injection point at time $t_{I}$ will then arrive at positions $s_{3}, s_{4}, s_{1}$, and $s_{2}$ at times

$$
\begin{gathered}
t_{3}=t_{I}+\left(s_{3}-s_{I}\right) / C \\
t_{4}=t_{I}+\left(s_{4}-s_{I}\right) / C \\
t_{1}=t_{I}+1-\left(s_{I}-s_{1}\right) / C \\
t_{2}=t_{I}+1-\left(s_{I}-s_{2}\right) / C
\end{gathered}
$$

where $C$ is the ring circumference. Eqs. (31) and (32) then become

$$
\begin{gathered}
\mathbf{X}_{a}=-\left(\mathbf{M}_{3 I}^{-1} \Phi_{3}\left(t_{3}\right)+\mathbf{M}_{4 I}^{-1} \Phi_{4}\left(t_{4}\right)\right) \\
X_{a}=b_{3 I} \phi_{3}\left(t_{3}\right)+b_{4 I} \phi_{4}\left(t_{4}\right) \\
X_{a}^{\prime}=-a_{3 I} \phi_{3}\left(t_{3}\right)-a_{4 I} \phi_{4}\left(t_{4}\right)
\end{gathered}
$$

and

$$
\begin{gathered}
\mathbf{X}_{b}=\mathbf{M}_{I 1} \Phi_{1}\left(t_{1}\right)+\mathbf{M}_{I 2} \Phi_{2}\left(t_{2}\right) \\
X_{b}=b_{I 1} \phi_{1}\left(t_{1}\right)+b_{I 2} \phi_{2}\left(t_{2}\right) \\
X_{b}^{\prime}=d_{I 1} \phi_{1}\left(t_{1}\right)+d_{I 2} \phi_{2}\left(t_{2}\right) .
\end{gathered}
$$

Using these equations in (38) and (39) we obtain the turn-by-turn positions and angles of a particle launched from the injection point.

Let us now consider the conditions under which the launched particle moves with the bump. Subtracting (55) from (58) we have

$$
\mathbf{X}_{b}-\mathbf{X}_{a}=\mathbf{M}_{I 1} \Phi_{1}\left(t_{1}\right)+\mathbf{M}_{I 2} \Phi_{2}\left(t_{2}\right)+\mathbf{M}_{3 I}^{-1} \Phi_{3}\left(t_{3}\right)+\mathbf{M}_{4 I}^{-1} \Phi_{4}\left(t_{4}\right)
$$

and

$$
\mathbf{M}_{4 I}\left(\mathbf{X}_{b}-\mathbf{X}_{a}\right)=\Phi_{4}\left(t_{4}\right)+\mathbf{M}_{43} \Phi_{3}\left(t_{3}\right)+\mathbf{M}_{42} \Phi_{2}\left(t_{2}\right)+\mathbf{M}_{41} \Phi_{1}\left(t_{1}\right) .
$$

If the bump is constant in time then it follows from (11) that the right-hand side of (62) is zero and we have

$$
\mathbf{X}_{a}=\mathbf{X}_{b}=\mathbf{X}_{c}
$$


where $\mathbf{X}_{c}$ is the constant closed orbit vector given by (12).

If the bump is collapsing then

$$
\Phi_{4}(t)+\mathbf{M}_{43} \Phi_{3}(t)+\mathbf{M}_{42} \Phi_{2}(t)+\mathbf{M}_{41} \Phi_{1}(t)=\mathbf{0}
$$

at each time $t$, but the right-hand side of (62) is generally not zero because the times $t_{j}$ are not all the same. We shall assume that $s_{3}-s_{I}, s_{4}-s_{I}$, $s_{I}-s_{1}$, and $s_{I}-s_{2}$ are small compared to the ring circumference, which allows the approximations

$$
t_{3} \approx t_{I}, \quad t_{4} \approx t_{I}, \quad t_{1} \approx t_{I}+1, \quad t_{2} \approx t_{I}+1 .
$$

It then follows that for a bump collapsing sufficiently slowly,

$$
\mathbf{M}_{3 I}^{-1} \Phi_{3}\left(t_{3}\right)+\mathbf{M}_{4 I}^{-1} \Phi_{4}\left(t_{4}\right) \approx \mathbf{M}_{3 I}^{-1} \Phi_{3}\left(t_{I}\right)+\mathbf{M}_{4 I}^{-1} \Phi_{4}\left(t_{I}\right)
$$

and

$$
\mathbf{M}_{I 1} \Phi_{1}\left(t_{1}\right)+\mathbf{M}_{I 2} \Phi_{2}\left(t_{2}\right) \approx \mathbf{M}_{I 1} \Phi_{1}\left(t_{I}+1\right)+\mathbf{M}_{I 2} \Phi_{2}\left(t_{I}+1\right) .
$$

Using

$$
\mathbf{M}_{4 I}^{-1} \Phi_{4}(t)+\mathbf{M}_{3 I}^{-1} \Phi_{3}(t)+\mathbf{M}_{I 2} \Phi_{2}(t)+\mathbf{M}_{I 1} \Phi_{1}(t)=0
$$

and

$$
\mathbf{X}_{c}(t)=\mathbf{M}_{I 2} \Phi_{2}(t)+\mathbf{M}_{I 1} \Phi_{1}(t)
$$

we then have

$$
\mathbf{X}_{a} \approx \mathbf{X}_{c}\left(t_{I}\right), \quad \mathbf{X}_{b} \approx \mathbf{X}_{c}\left(t_{I}+1\right)
$$

Finally, using this in (30) we have

$$
\mathbf{Y}-\mathbf{X}_{c}\left(t_{I}+1\right) \approx \mathbf{M}\left\{\mathbf{X}-\mathbf{X}_{c}\left(t_{I}\right)\right\}
$$

which shows that the injected beam moves with the closed orbit if the bump is collapsed sufficiently slowly.

\section{Non-closure of the Collapsing Bump}

Now let

$$
\begin{aligned}
& \tau_{1}=t_{I}+1-\left(s_{I}-s_{1}\right) / C \\
& \tau_{2}=t_{I}+1-\left(s_{I}-s_{2}\right) / C \\
& \tau_{3}=t_{I}+1+\left(s_{3}-s_{I}\right) / C
\end{aligned}
$$




$$
\tau_{4}=t_{I}+1+\left(s_{4}-s_{I}\right) / C
$$

and consider a particle launched from $s_{1}$ with zero position and with angle $\phi_{1}\left(\tau_{1}\right)$. Then the position and angle at the injection point $s_{I}$ are given by

$$
\mathbf{X}_{b}=\mathbf{M}_{I 1} \Phi_{1}\left(\tau_{1}\right)+\mathbf{M}_{I 2} \Phi_{2}\left(\tau_{2}\right)
$$

and at $s_{3}$ we have

$$
\mathbf{X}_{d}=\mathbf{M}_{3 I} \mathbf{X}_{b}+\Phi_{3}\left(\tau_{3}\right) .
$$

The position and angle at $s_{4}$ are then given by

$$
\begin{gathered}
\mathbf{X}_{e}=\mathbf{M}_{43} \mathbf{X}_{d}+\Phi_{4}\left(\tau_{4}\right) \\
\mathbf{X}_{e}=\mathbf{M}_{4 I} \mathbf{X}_{b}+\mathbf{M}_{43} \Phi\left(\tau_{3}\right)+\Phi_{4}\left(\tau_{4}\right)
\end{gathered}
$$

and

$$
\mathbf{X}_{e}=\Phi_{4}\left(\tau_{4}\right)+\mathbf{M}_{43} \Phi_{3}\left(\tau_{3}\right)+\mathbf{M}_{42} \Phi_{2}\left(\tau_{2}\right)+\mathbf{M}_{41} \Phi_{1}\left(\tau_{1}\right) .
$$

This is the amount by which the orbit does not close at $s_{4}$. If the bump is constant in time then the right-hand side of (80) is zero and the orbit is closed. For a collapsing bump the right-hand side is generally non-zero.

Writing

$$
\mathbf{X}_{e}=\left(\begin{array}{c}
X_{e} \\
X_{e}^{\prime}
\end{array}\right)
$$

and using

$$
\mathbf{M}_{4 k} \Phi_{k}=\left(\begin{array}{cc}
a_{4 k} & b_{4 k} \\
c_{4 k} & d_{4 k}
\end{array}\right)\left(\begin{array}{c}
0 \\
\phi_{k}
\end{array}\right)=\left(\begin{array}{c}
b_{4 k} \\
d_{4 k}
\end{array}\right) \phi_{k}
$$

we have

$$
\begin{gathered}
X_{e}=b_{41} \phi_{1}\left(\tau_{1}\right)+b_{42} \phi_{2}\left(\tau_{2}\right)+b_{43} \phi_{3}\left(\tau_{3}\right) \\
X_{e}^{\prime}=d_{41} \phi_{1}\left(\tau_{1}\right)+d_{42} \phi_{2}\left(\tau_{2}\right)+d_{43} \phi_{3}\left(\tau_{3}\right)+\phi_{4}\left(\tau_{4}\right) .
\end{gathered}
$$

The emittance associated with $\mathbf{X}_{e}$ is

$$
\epsilon_{e}=\gamma_{4} X_{e}^{2}+2 \alpha_{4} X_{e} X_{e}^{\prime}+\beta_{4} x_{e}^{2}
$$

where $\alpha_{4}, \beta_{4}$, and $\gamma_{4}$ are the Courant-Snyder lattice parameters at $s_{4}$.

Since $s_{3}-s_{I}, s_{4}-s_{I}, s_{I}-s_{1}$, and $s_{I}-s_{2}$ are assumed small compared to the ring circumference we have

$$
\tau_{1} \approx \tau_{2} \approx \tau_{3} \approx \tau_{4} \approx \tau
$$


where $\tau=t_{I}+1$. If the bump collapses sufficiently slowly we then have

$$
\mathbf{X}_{e} \approx \Phi_{4}(\tau)+\mathbf{M}_{43} \Phi_{3}(\tau)+\mathbf{M}_{42} \Phi_{2}(\tau)+\mathbf{M}_{41} \Phi_{1}(\tau)
$$

and we see that $\mathbf{X}_{e}$ and $\epsilon_{e}$ are close to zero.

On the other hand, if the bump collapses rapidly to zero just after time $t_{I}+1$ then

$$
\phi_{3}\left(\tau_{3}\right)=0, \quad \phi_{4}\left(\tau_{4}\right)=0
$$

and (80) becomes

$$
\mathbf{X}_{e}=\mathbf{M}_{42} \Phi_{2}\left(\tau_{2}\right)+\mathbf{M}_{41} \Phi_{1}\left(\tau_{1}\right)
$$

In this case we have

$$
\mathbf{M}_{42} \Phi_{2}\left(\tau_{2}\right)+\mathbf{M}_{41} \Phi_{1}\left(\tau_{1}\right) \approx \mathbf{M}_{42} \Phi_{2}\left(t_{I}+1\right)+\mathbf{M}_{41} \Phi_{1}\left(t_{I}+1\right)
$$

and

$$
\mathbf{X}_{e} \approx \mathbf{M}_{4 I} \mathbf{X}_{c}\left(t_{I}+1\right)
$$

which shows that $\mathbf{X}_{e}$ is as large as the bump itself.

\section{Turn-by-Turn Evolution of the Beam Ellipse}

The incoming beam ellipse at the point of injection is given by

$$
\gamma_{0}\left(X-X_{0}\right)^{2}+2 \alpha_{0}\left(X-X_{0}\right)\left(X^{\prime}-X_{0}^{\prime}\right)+\beta_{0}\left(X^{\prime}-X_{0}^{\prime}\right)^{2}=\epsilon_{0}
$$

where $X$ and $X^{\prime}$ are the position and angle of a beam particle on the ellipse, $X_{0}$ and $X_{0}^{\prime}$ are the position and angle of the center of the ellipse, and $\pi \epsilon_{0}$ is the area of the ellipse. As discussed in [2,3], the initial ellipse parameters $\alpha_{0}, \beta_{0}$, and $\gamma_{0}$ are taken to be

$$
\alpha_{0}=B \alpha_{I}, \quad \beta_{0}=B \beta_{I}, \quad \gamma_{0}=\frac{1+\alpha_{0}^{2}}{\beta_{0}}
$$

where the parameter $B$ is adjusted to make optimum use of the machine and injection inflector acceptances. Optimum use of the machine acceptance also requires that

$$
X_{0}^{\prime}-X_{c}^{\prime}=-\alpha_{I}\left(X_{0}-X_{c}\right) / \beta_{I}
$$


and

$$
X_{0}=X_{s}+\sqrt{\epsilon_{0} \beta_{0}}
$$

where $X_{s}$ is the position of the outer side of the inflector septum. Eq. (94) is satisfied if we take

$$
X_{0}^{\prime}=-\alpha_{I} X_{0} / \beta_{I}, \quad X_{c}^{\prime}=-\alpha_{I} X_{c} / \beta_{I}
$$

Writing

$$
\mathbf{X}=\left(\begin{array}{c}
X \\
X^{\prime}
\end{array}\right), \quad \mathbf{X}_{0}=\left(\begin{array}{c}
X_{0} \\
X_{0}^{\prime}
\end{array}\right)
$$

and

$$
\mathbf{E}_{0}=\left(\begin{array}{rr}
\beta_{0} & -\alpha_{0} \\
-\alpha_{0} & \gamma_{0}
\end{array}\right), \quad \mathbf{E}_{0}^{-1}=\left(\begin{array}{cc}
\gamma_{0} & \alpha_{0} \\
\alpha_{0} & \beta_{0}
\end{array}\right)
$$

the equation for the beam ellipse becomes

$$
\left(\mathbf{X}^{T}-\mathbf{X}_{0}^{T}\right) \mathbf{E}_{0}^{-1}\left(\mathbf{X}-\mathbf{X}_{0}\right)=\epsilon_{0}
$$

where the superscript $T$ denotes the transpose of a vector or matrix. After one turn around the machine we have

$$
\begin{gathered}
\mathbf{Y}=\mathbf{M X}+\mathbf{M}_{I 1} \mathbf{N}\left(\mathbf{M}_{43} \Phi_{3}+\Phi_{4}\right)+\mathbf{M}_{I 1} \Phi_{1}+\mathbf{M}_{I 2} \Phi_{2} \\
\mathbf{Y}_{0}=\mathbf{M X}_{0}+\mathbf{M}_{I 1} \mathbf{N}\left(\mathbf{M}_{43} \Phi_{3}+\Phi_{4}\right)+\mathbf{M}_{I 1} \Phi_{1}+\mathbf{M}_{I 2} \Phi_{2} \\
\mathbf{Y}-\mathbf{Y}_{0}=\mathbf{M}\left(\mathbf{X}-\mathbf{X}_{0}\right)
\end{gathered}
$$

and it follows that

$$
\left(\mathbf{Y}^{T}-\mathbf{Y}_{0}^{T}\right) \mathbf{F}^{-1}\left(\mathbf{Y}-\mathbf{Y}_{0}\right)=\epsilon_{0}
$$

where

$$
\mathbf{F}=\mathbf{M E}_{0} \mathbf{M}^{T} .
$$

This gives the turn-by-turn evolution of the beam ellipse.

\section{$6 \quad$ The Injection Dipoles}

The four injection dipoles are ferrite-core magnets located inside the vacuum chamber in the C1, C3, C7, and D1 straights. Specifically, the locations are as follows: 
1. The $\mathrm{C} 1$ injection dipole is just downstream of main dipole DHB8 and just upstream of vertical dipole corrector DVCC1;

2. The C3 injection dipole is just downstream of quadrupole QVC3;

3. The $\mathrm{C} 7$ injection dipole is just downstream of the $\mathrm{C} 6$ current transformer and just upstream of corrector DVCC7;

4. The D1 injection dipole is just downstream of main dipole DHC8 and just upstream of corrector DVCD1.

Note that the exit of the C3 inflector is 2.756936 meters downstream of the center of the C3 injection dipole and 0.217555 meters upstream of horizontal dipole corrector DHCC4. (These numbers come from the MAD input file for Booster.)

The H-minus injection foil is downstream of main dipole DHC5 and upstream of corrector DHCC6. The sequence of elements downstream of the injection foil is DHCC6, SHC6 (sextuple), PUEHC6 (pick-up electrode), QHC6, LC6 (drift), C6 Current Transformer, C7 injection dipole, and DVCC7.

As per Ref. [4] each injection dipole has the following properties:

1. The integrated strength at $1200 \mathrm{~A}$ is $0.016 \mathrm{Tm}$ which gives $1.33 \times 10^{-5} \mathrm{Tm} / \mathrm{A}$;

2. The peak magnetic field at $1200 \mathrm{~A}$ is 852 Gauss;

3. The average magnetic field over a magnetic length of $45.7 \mathrm{~cm}$ is 351 Gauss (at 1200 A);

4. The vertical gap is 2.73 inches $(6.93 \mathrm{~cm})$;

5. The horizontal width is 4.25 inches $(10.80 \mathrm{~cm})$;

6. The physical length is 5.99 inches $(15.21 \mathrm{~cm})$.

The power supplies for the injection dipoles provide programmed currents by discharging a capacitor bank through a transistor bank. Experience has shown that the transistors can be damaged if the requested current and voltage are too high. To prevent this, the rate of change of current is limited to about $6 \mathrm{~A} / \mu \mathrm{s}$. This is a factor of 2 less than the $1200 \mathrm{~A}$ per $100 \mu$ s advertised in Ref. [5]. In the next section we will see that by 
judiciously choosing the horizontal tune and the initial distance of the orbit from the septum one can stay well within the $6 \mathrm{~A} / \mu$ s limit and inject up to 4 turns without loss on the septum.

\section{Illustration of the Injection Process}

The injection process is illustrated with several examples in Figures $\mathbf{1}$ through 49. In each example the injection bump position $X_{c}$ starts at some initial value and then falls linearly to zero over a period of several turns. A beam ellipse is launched from the injection point at various times during the bump fall and is tracked turn-by-turn around the machine. The magnetic rigidity of the beam is taken to be $1.255 \mathrm{Tm}$ which is close to the maximum expected in the ETB (EBIS-to-Booster) transport line.

Figures 1 through 14 show that with horizontal tune $Q_{H}=4.75$ and with initial bump position $X_{c}=30 \mathrm{~mm}$, one can inject 4 turns of EBIS beam with no loss on the inflector septum. The required maximum rate of fall of the injection dipole currents is $4.19 \mathrm{~A} / \mu \mathrm{s}$.

Figures 15 through 29 show that with horizontal tune $Q_{H}=4.74$ and with initial bump position $X_{c}=30 \mathrm{~mm}$, one can again inject 4 turns of EBIS beam with no loss on the inflector septum. The required maximum rate of fall of the injection dipole currents in this case is $4.47 \mathrm{~A} / \mu \mathrm{s}$.

In Figures 30 through $\mathbf{3 7}$ the horizontal tune is raised to 4.80. The initial bump position is lowered to $X_{c}=20 \mathrm{~mm}$ in order to keep the injected beam from hitting the septum. In this case one can inject just 2 turns without loss on the septum. The maximum rate of fall of the injection dipole currents is $4.16 \mathrm{~A} / \mu \mathrm{s}$.

In Figures 38 through 49 the horizontal tune is lowered to 4.70. This allows the initial bump position to be raised to $X_{c}=36 \mathrm{~mm}$ without getting loss on the septum. In this case one can inject 4 turns without loss on the septum, but the maximum rate of fall of the injection dipole currents increases to $6.87 \mathrm{~A} / \mu \mathrm{s}$. 


\section{References}

[1] C. J. Gardner, "Booster Inflector Specifications", Booster Tech. Note 159, February 28, 1990.

[2] C.J. Gardner, "Multiturn Injection of Heavy Ions into the Booster", Booster Tech. Note 197, August 14, 1991.

[3] C.J. Gardner, "Notes on the Injection of EBIS Ions into Booster", C-A/AP/Note 240, June 2006.

[4] Memo from S.Y. Zhang and C. Eld, "Booster Injection Kicker Magnet Test", February 14, 1991.

[5] Memo from S.Y. Zhang, "Booster Injection Kicker Operation", January 7, 1992. 


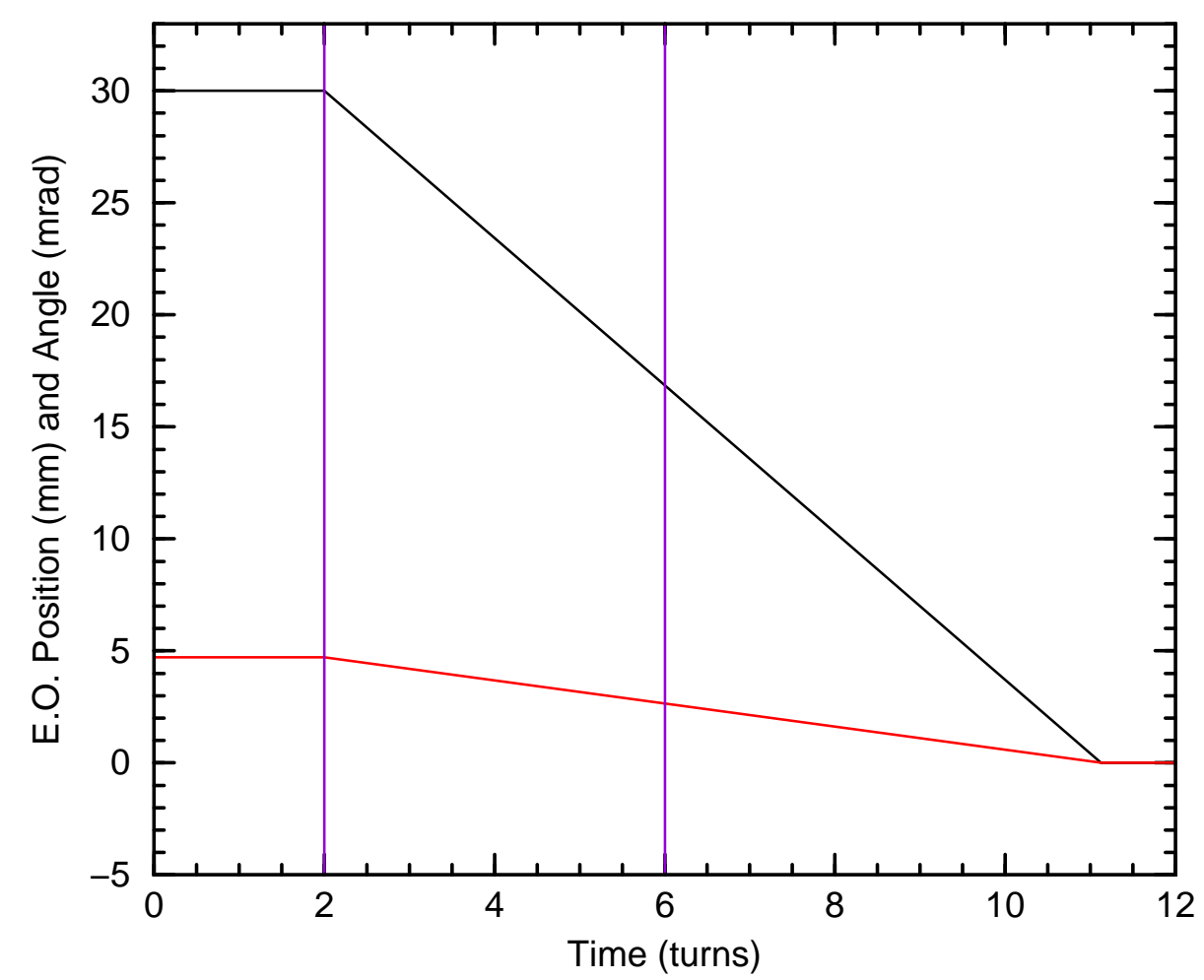

Figure 1: Here the black and red curves are the injection bump position $X_{c}$ and angle $X_{c}^{\prime}=-\alpha_{I} X_{c} / \beta_{I}$ as functions of time for four-turn injection of EBIS beams. The time axis is marked in turns, where one turn is 10.276 $\mu \mathrm{s}$. The beam pulse occurs between the two violet lines. The horizontal and vertical tunes are $Q_{H}=4.75$ and $Q_{V}=4.81$, and the MAD program gives $\alpha_{I}=-1.733$ and $\beta_{I}=11.033 \mathrm{~m}$. The magnetic rigidity is taken to be 1.255 Tm which is close to the expected maximum for EBIS beams. 


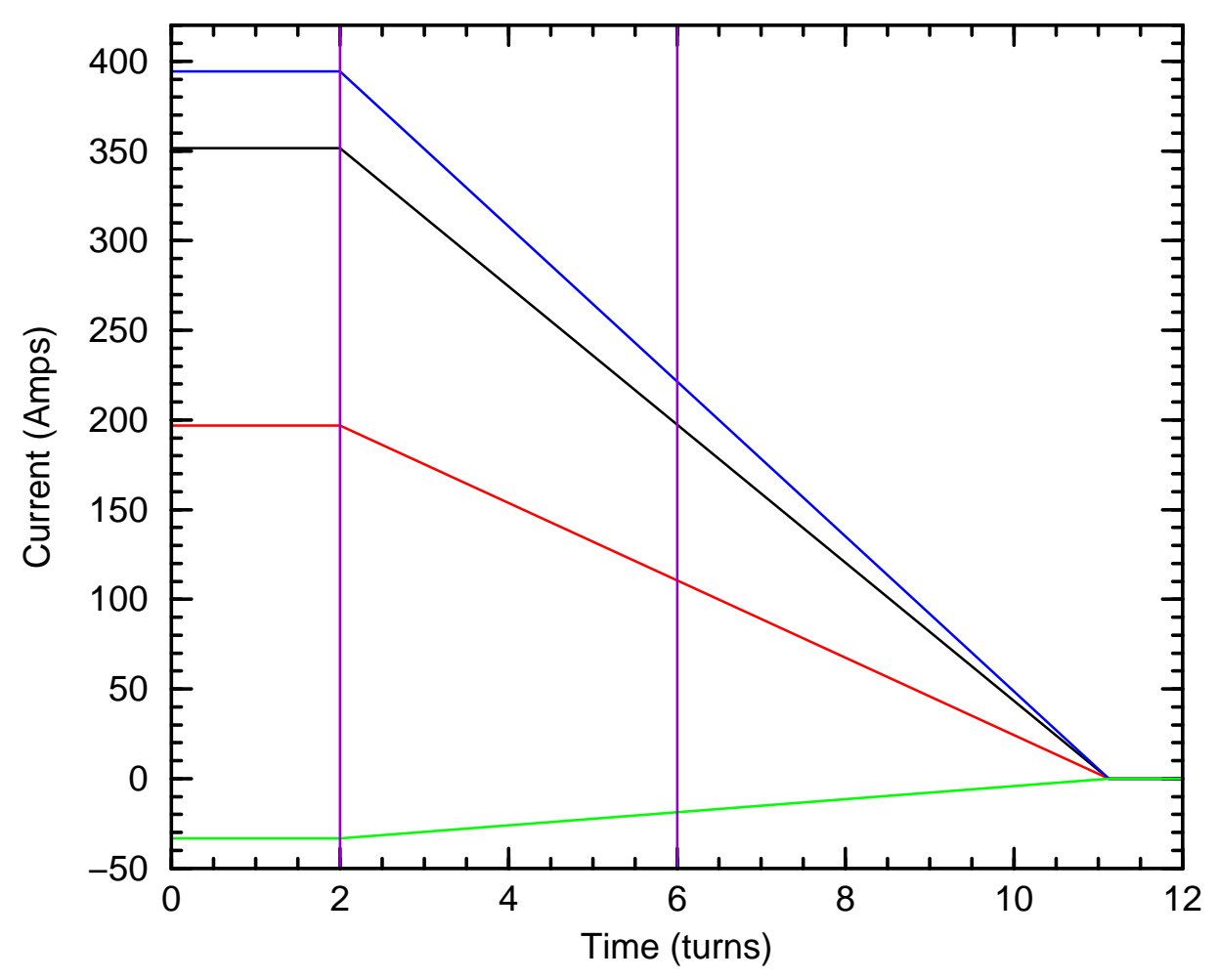

Figure 2: Here the black, red, blue, and green curves are the C1, C3, C7, and D1 dipole currents, respectively, for the injection bump function of Figure 1. The four-turn EBIS beam pulse occurs between the two violet lines. The rate of fall of the $\mathrm{C} 7$ dipole current (blue curve) is $4.19 \mathrm{~A} / \mu \mathrm{s}$. 


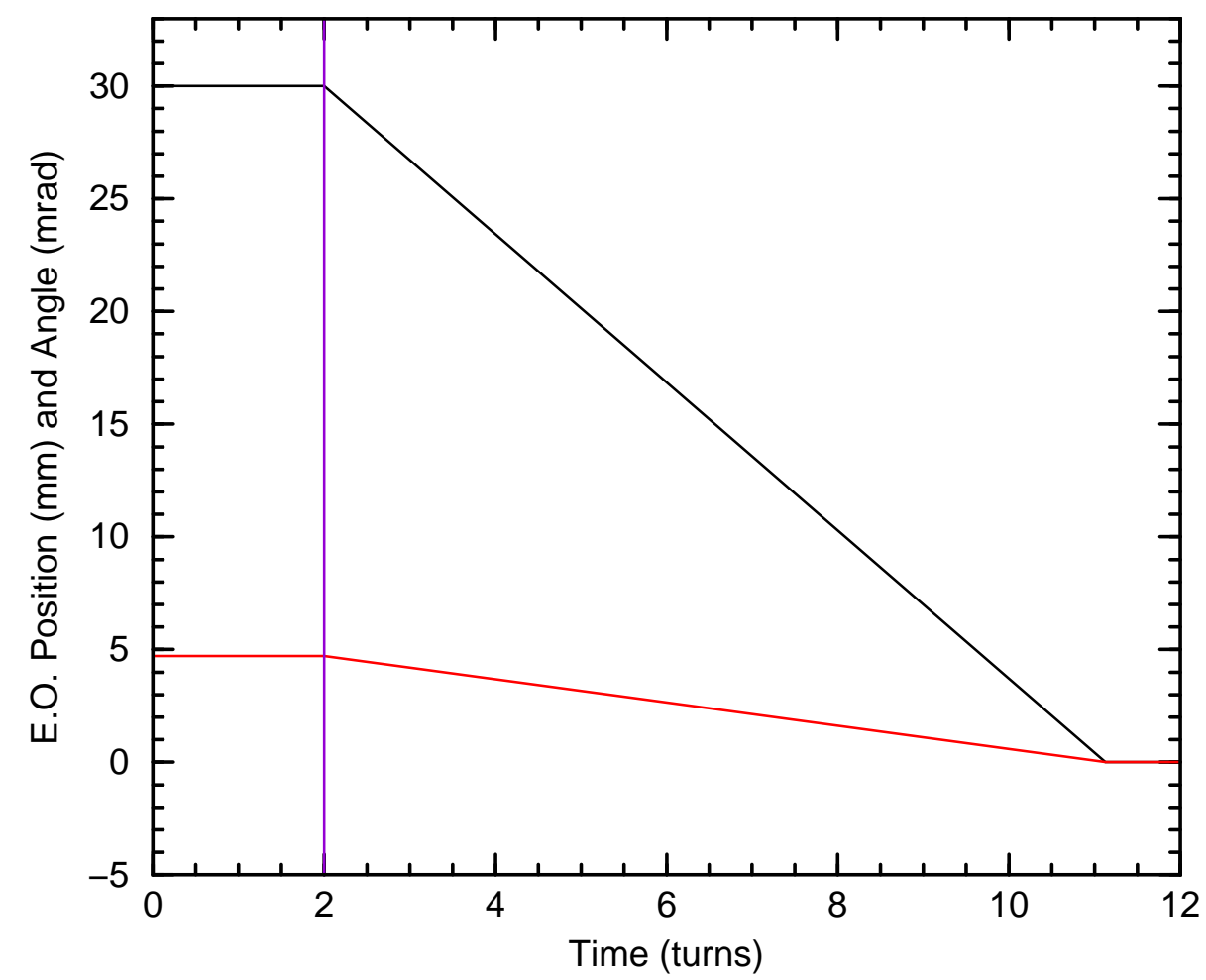

Figure 3: We consider the evolution of a beam ellipse launched from the injection point (i.e. the exit of the $\mathrm{C} 3$ inflector) at time 2.0 turns as indicated by the violet line above. Figures 4 through $\mathbf{7}$ show the evolution of the beam ellipse. The necessary computations were carried out with Fortran program "EBinject" using MAD Twiss file TwissEBinjct75. 


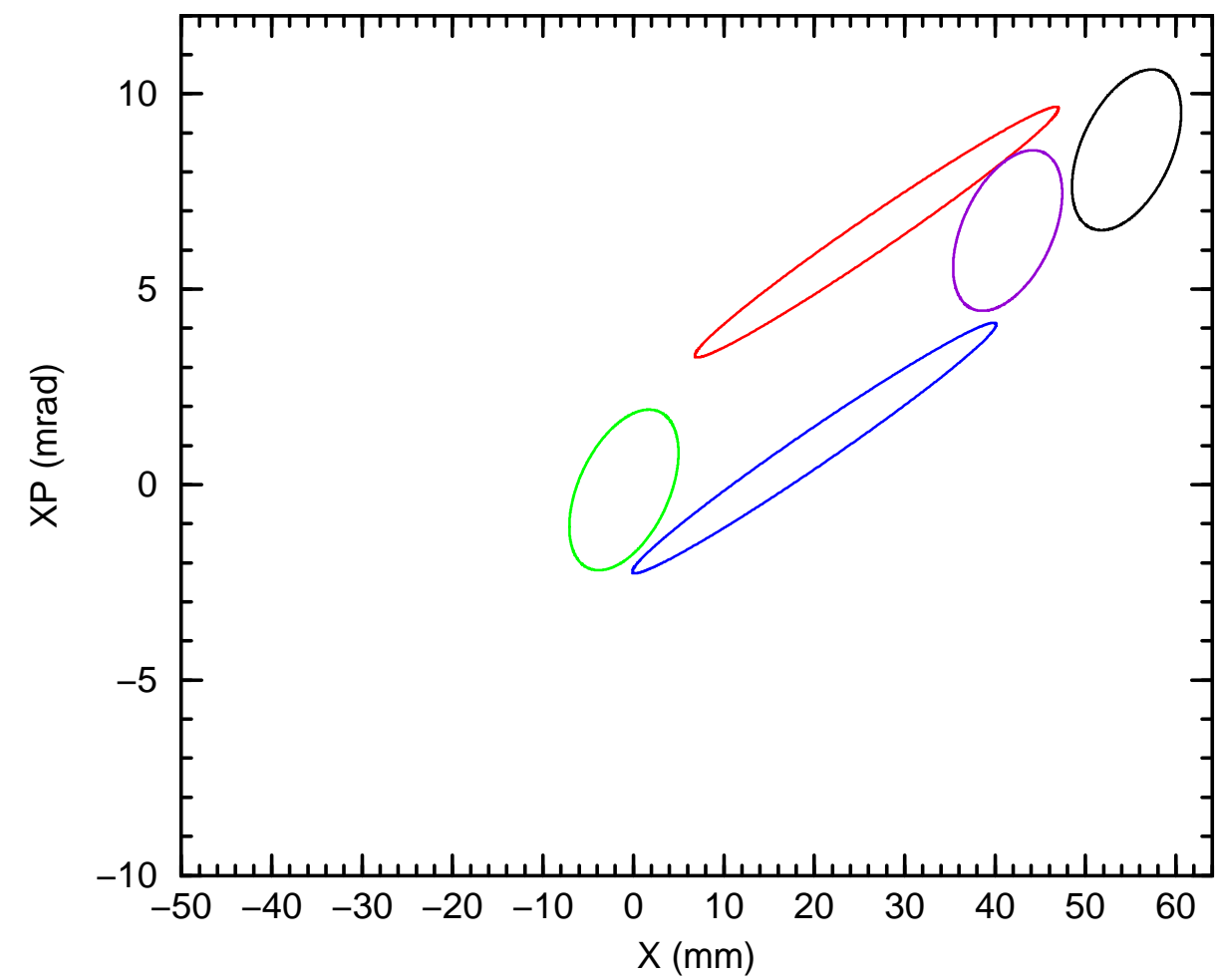

Figure 4: The black, red, green, blue, and violet curves are the beam ellipse after $0,1,2,3$, and 4 turns, respectively, from launch time 2.0 turns. The initial (black) ellipse has parameter $B=0.3$ and area $\pi \epsilon_{0}=11.0 \pi \mathrm{mm}$ milliradians. The outer side of the $1 \mathrm{~mm}$ thick inflector septum is located at $X=48.5 \mathrm{~mm}$. The tunes are $Q_{H}=4.75$ and $Q_{V}=4.81$. 


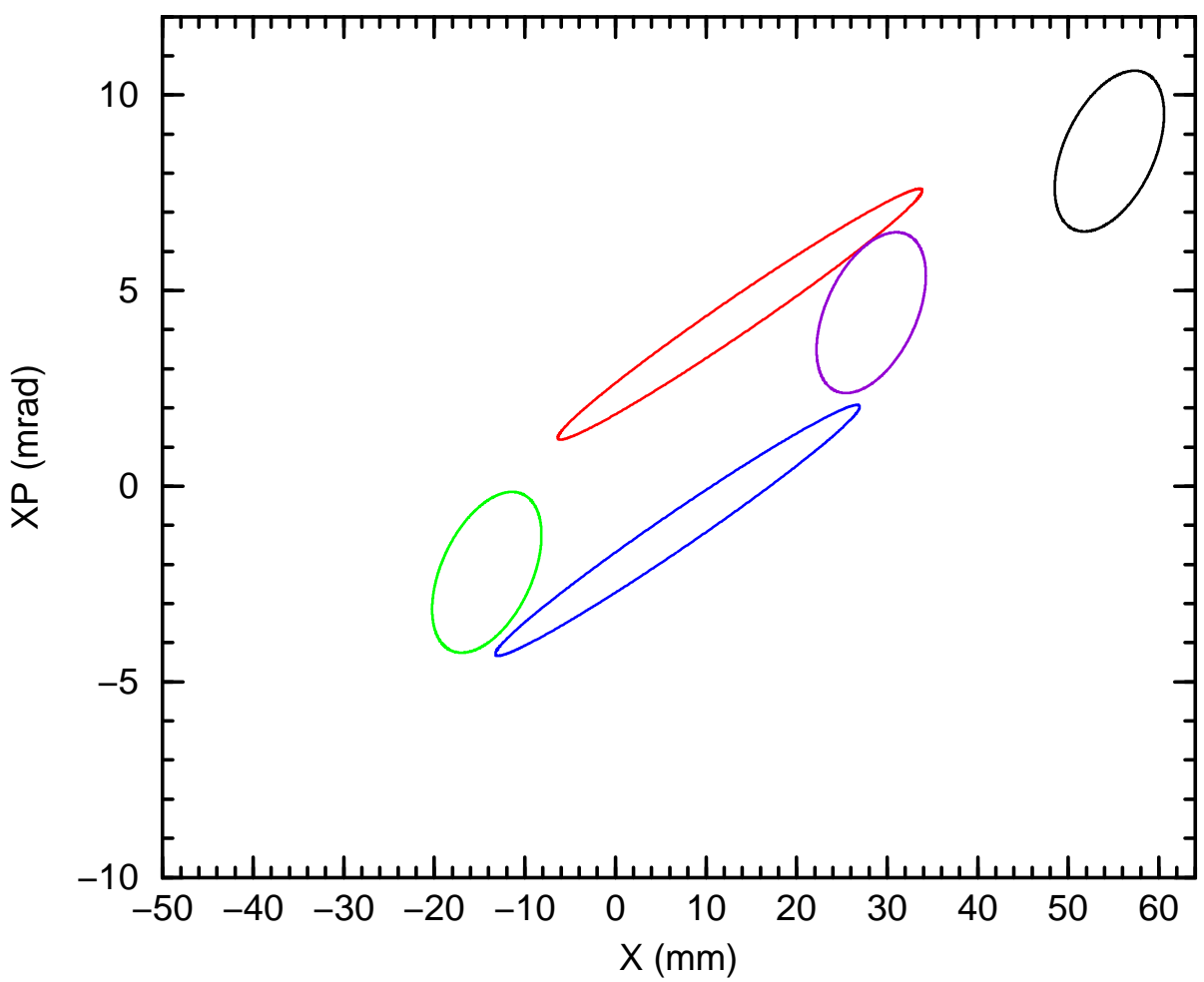

Figure 5: The black, red, green, blue, and violet curves are the beam ellipse after $0,5,6,7$, and 8 turns, respectively, from launch time 2.0 turns. Note that the injected ellipse moves with the falling bump. 


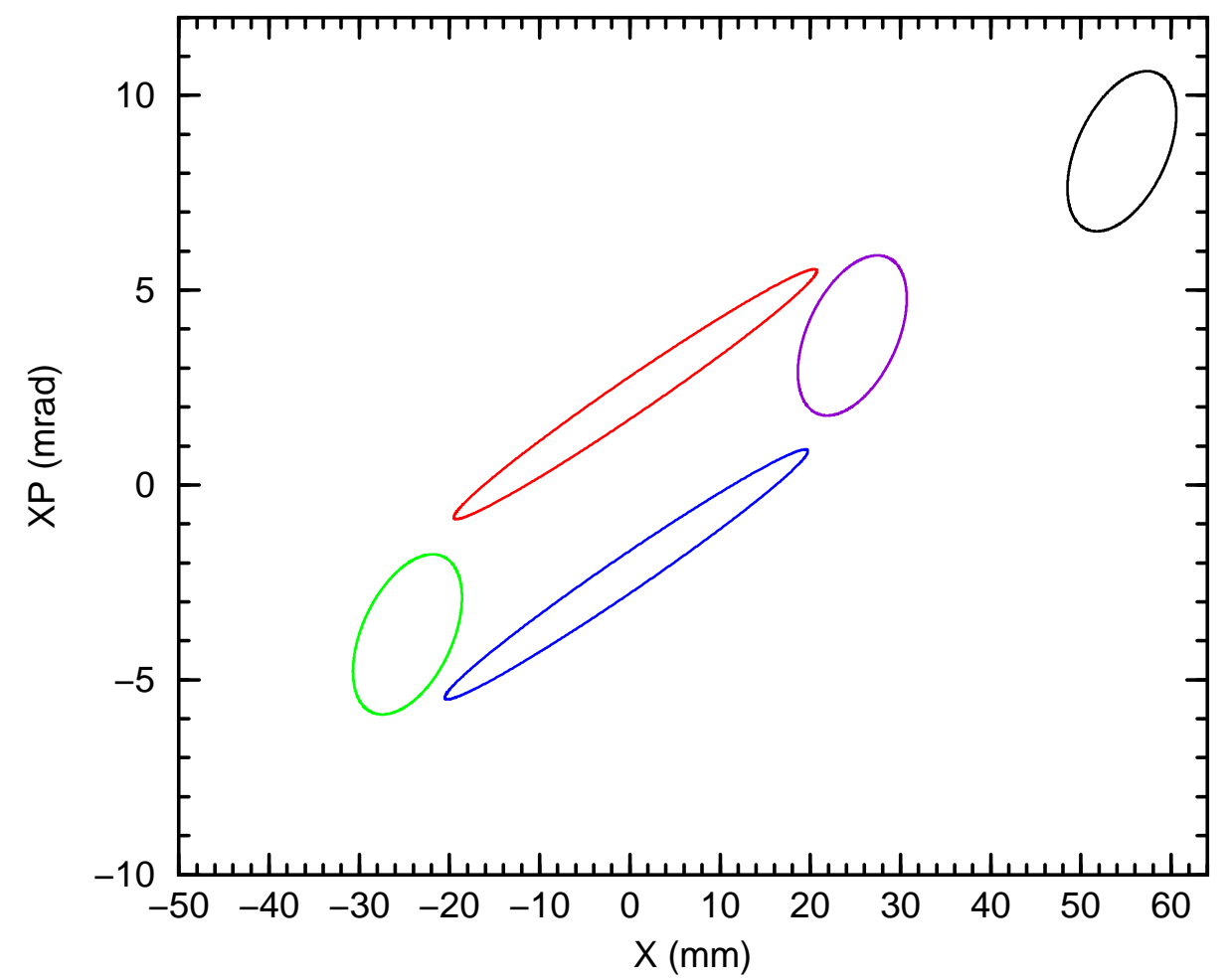

Figure 6: The black, red, green, blue, and violet curves are the beam ellipse after $0,9,10,11$, and 12 turns, respectively, from launch time 2.0 turns. 


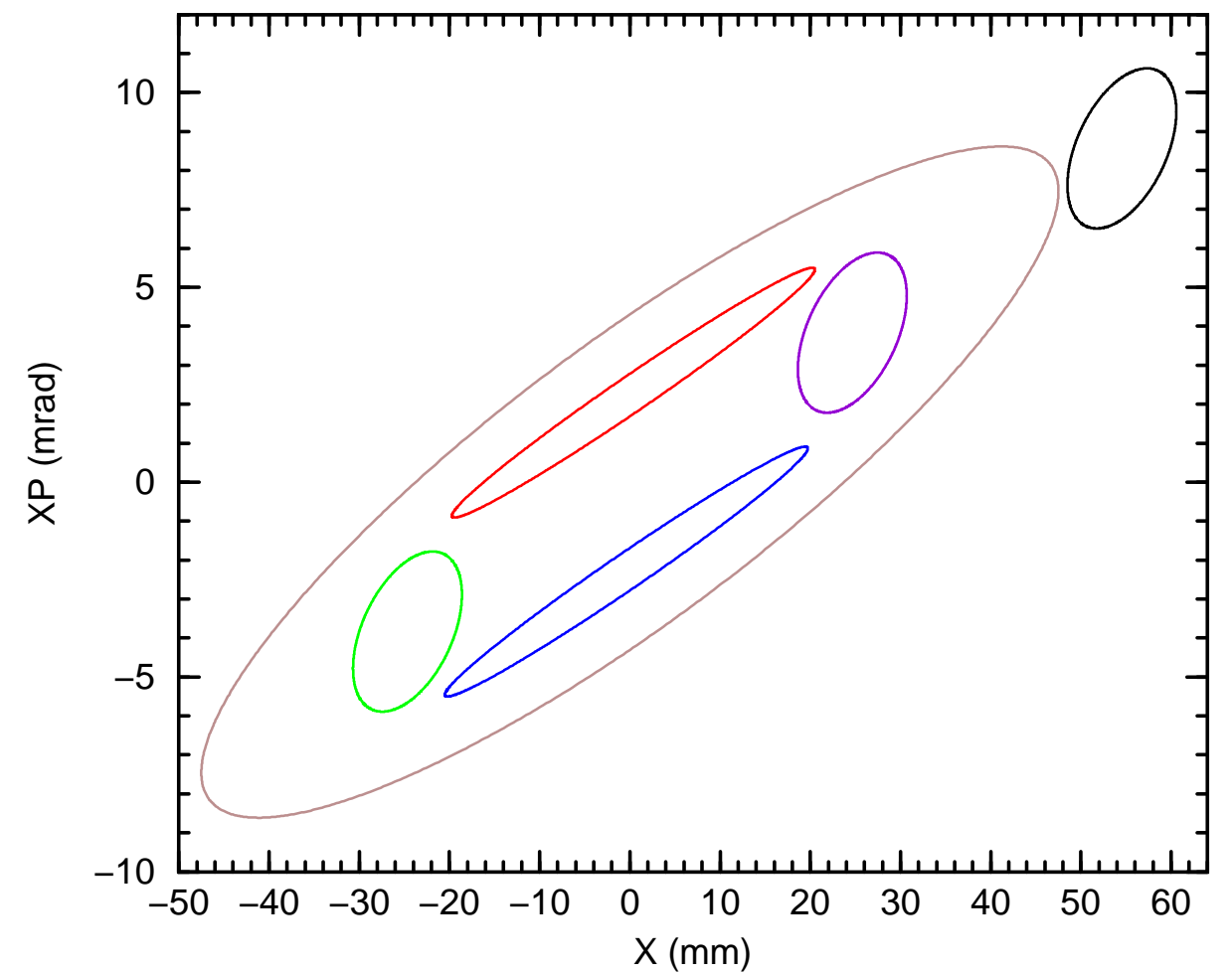

Figure 7: The black, red, green, blue, and violet curves are the beam ellipse after $0,13,14,15$, and 16 turns, respectively, from launch time 2.0 turns. The large brown ellipse is the machine acceptance, taken to be $204.5 \pi \mathrm{mm}$ milliradians. The initial (black) ellipse launched at time 2.0 turns therefore ends up well inside the machine acceptance after the bump has collapsed. 


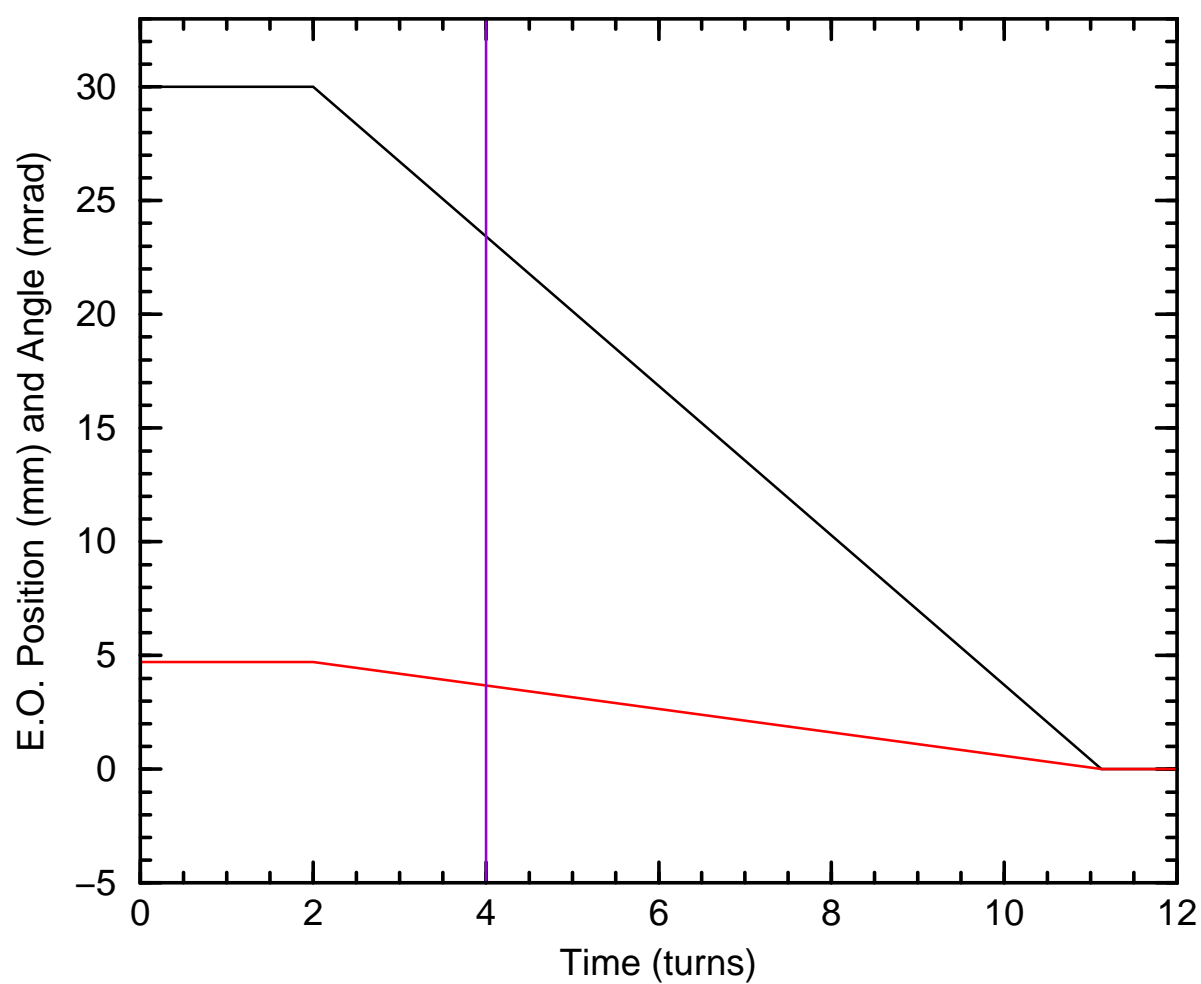

Figure 8: Now consider the evolution of a beam ellipse launched from the injection point at time 4.0 turns as indicated by the violet line above. Figures 9 through 11 show the evolution of the beam ellipse. As before, the necessary computations were carried out with Fortran program "EBinject" using MAD Twiss file TwissEBinjct75. 


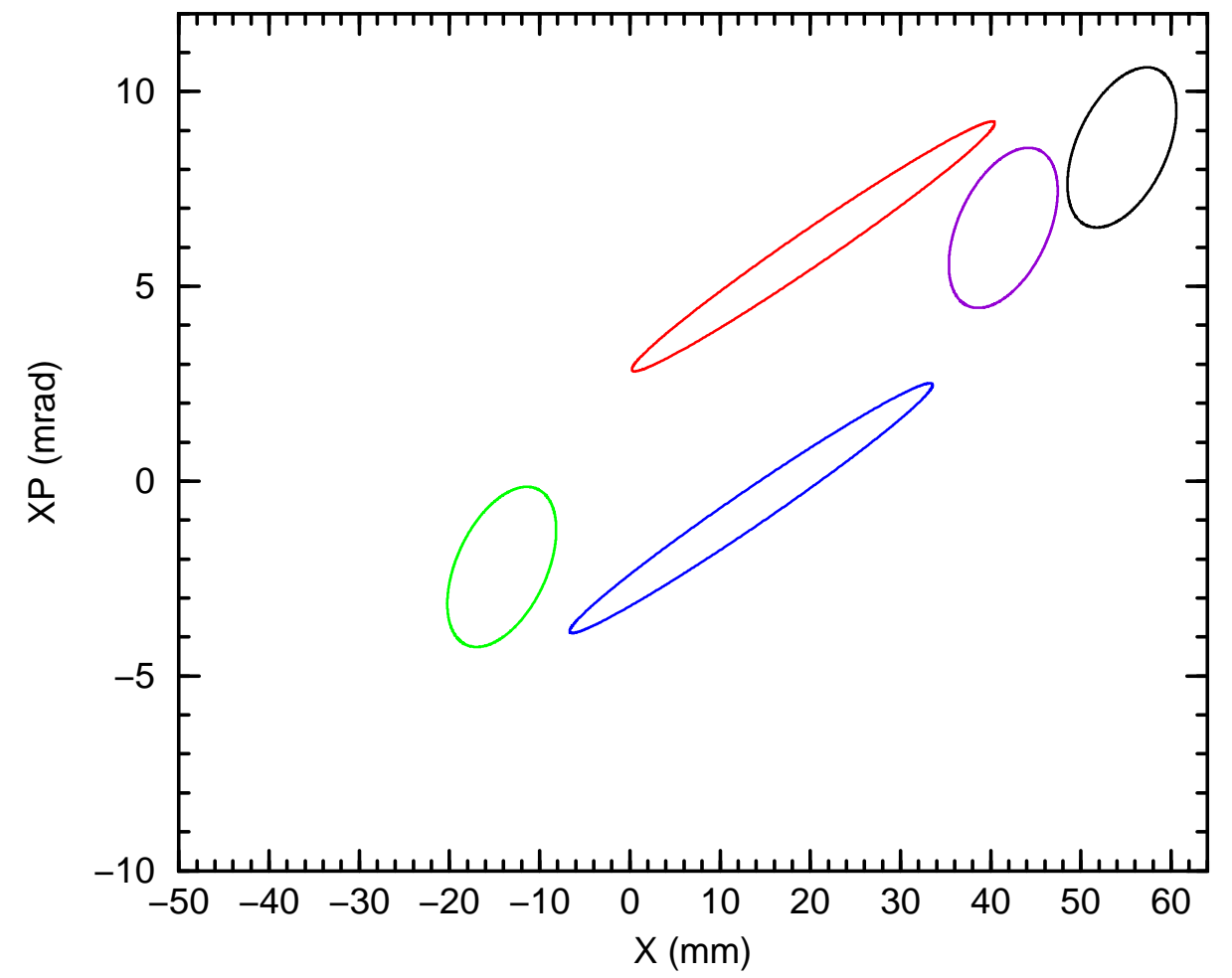

Figure 9: The black, red, green, blue, and violet curves are the beam ellipse after $0,1,2,3$, and 4 turns, respectively, from launch time 4.0 turns. As before, the initial (black) ellipse has parameter $B=0.3$ and area $\pi \epsilon_{0}=$ $11.0 \pi \mathrm{mm}$ milliradians. The outer side of the $1 \mathrm{~mm}$ thick inflector septum is located at $X=48.5 \mathrm{~mm}$. The tunes are $Q_{H}=4.75$ and $Q_{V}=4.81$. 


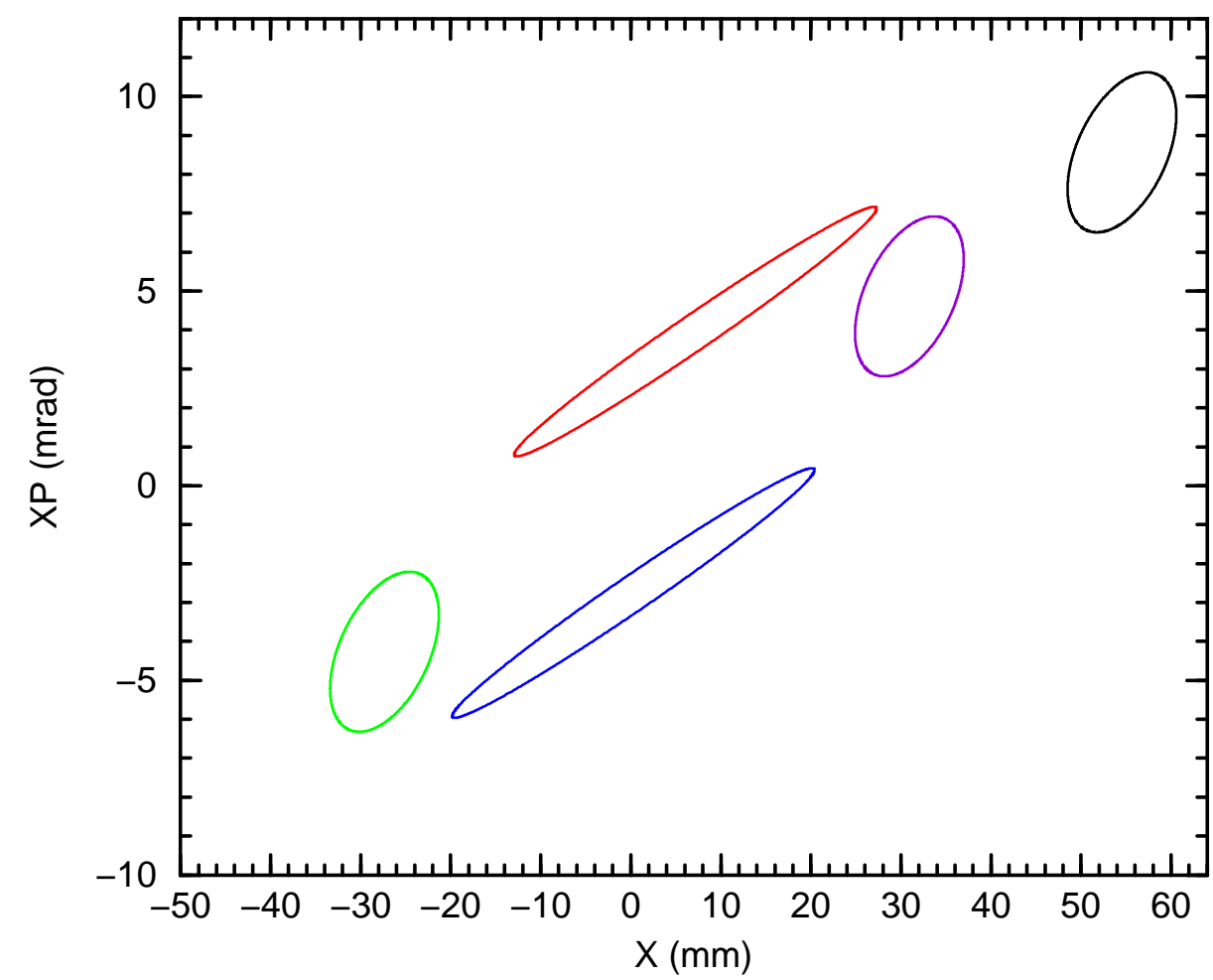

Figure 10: The black, red, green, blue, and violet curves are the beam ellipse after $0,5,6,7$, and 8 turns, respectively, from launch time 4.0 turns. Note again that the injected ellipse moves with the falling bump. 


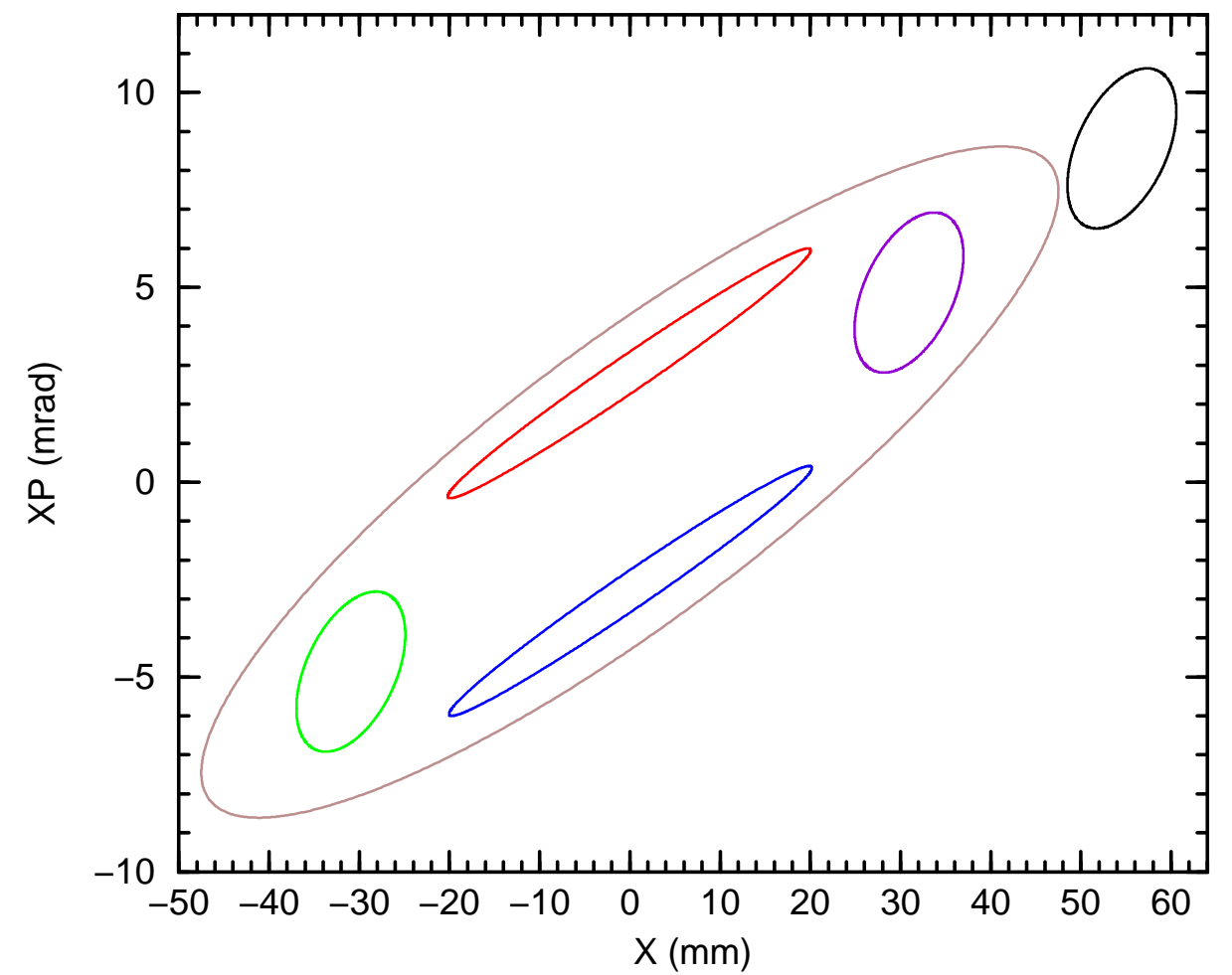

Figure 11: The black, red, green, blue, and violet curves are the beam ellipse after $0,9,10,11$, and 12 turns, respectively, from launch time 4.0 turns. The large brown ellipse is the machine acceptance, taken to be $204.5 \pi \mathrm{mm}$ milliradians. The initial (black) ellipse launched at time 4.0 turns therefore ends up well inside the machine acceptance after the bump has collapsed. 


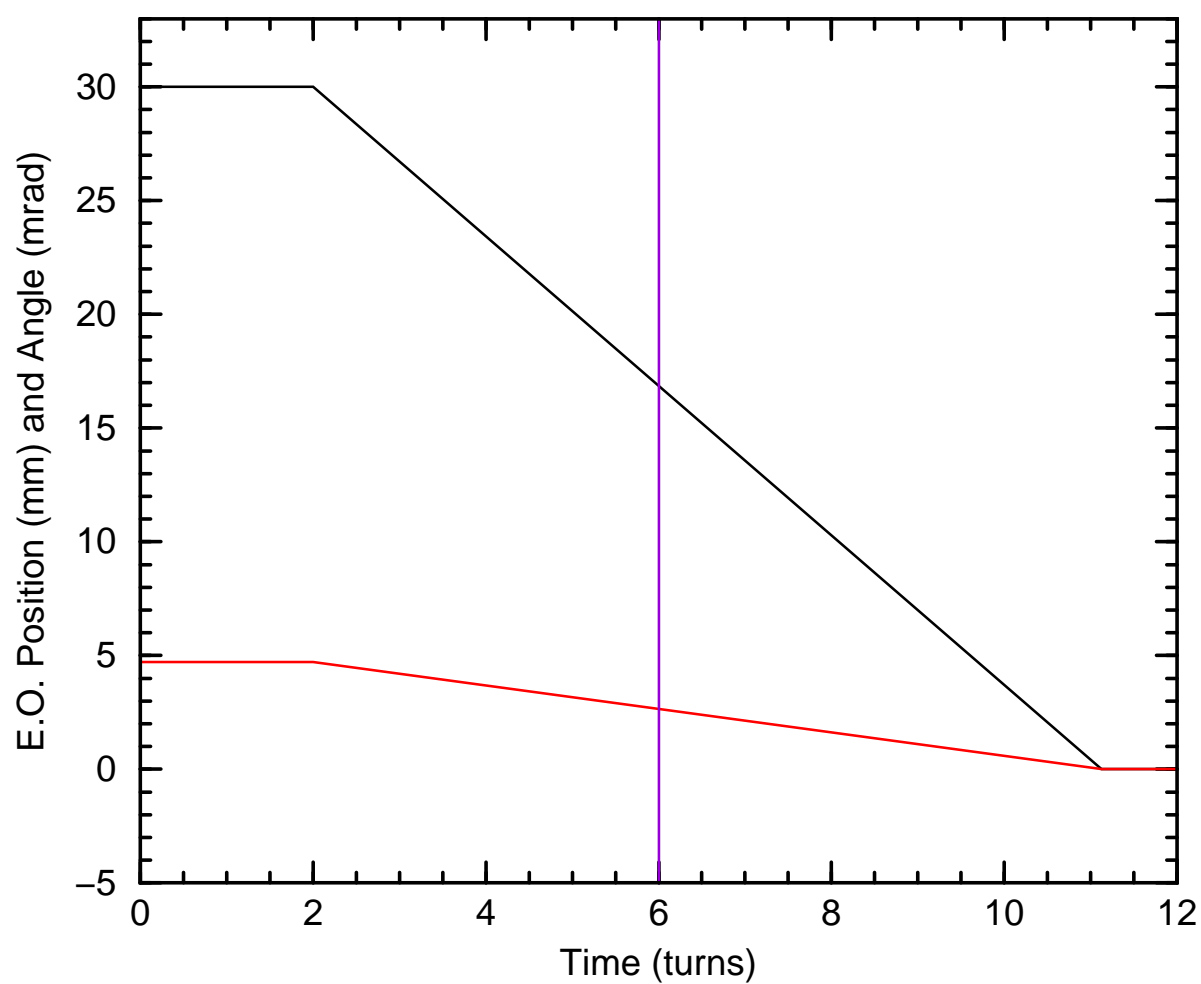

Figure 12: Now consider the evolution of a beam ellipse launched from the injection point at time 6.0 turns as indicated by the violet line above. Figures 13 and 14 show the evolution of the beam ellipse. As before, the necessary computations were carried out with Fortran program "EBinject" using MAD Twiss file TwissEBinjct75. 


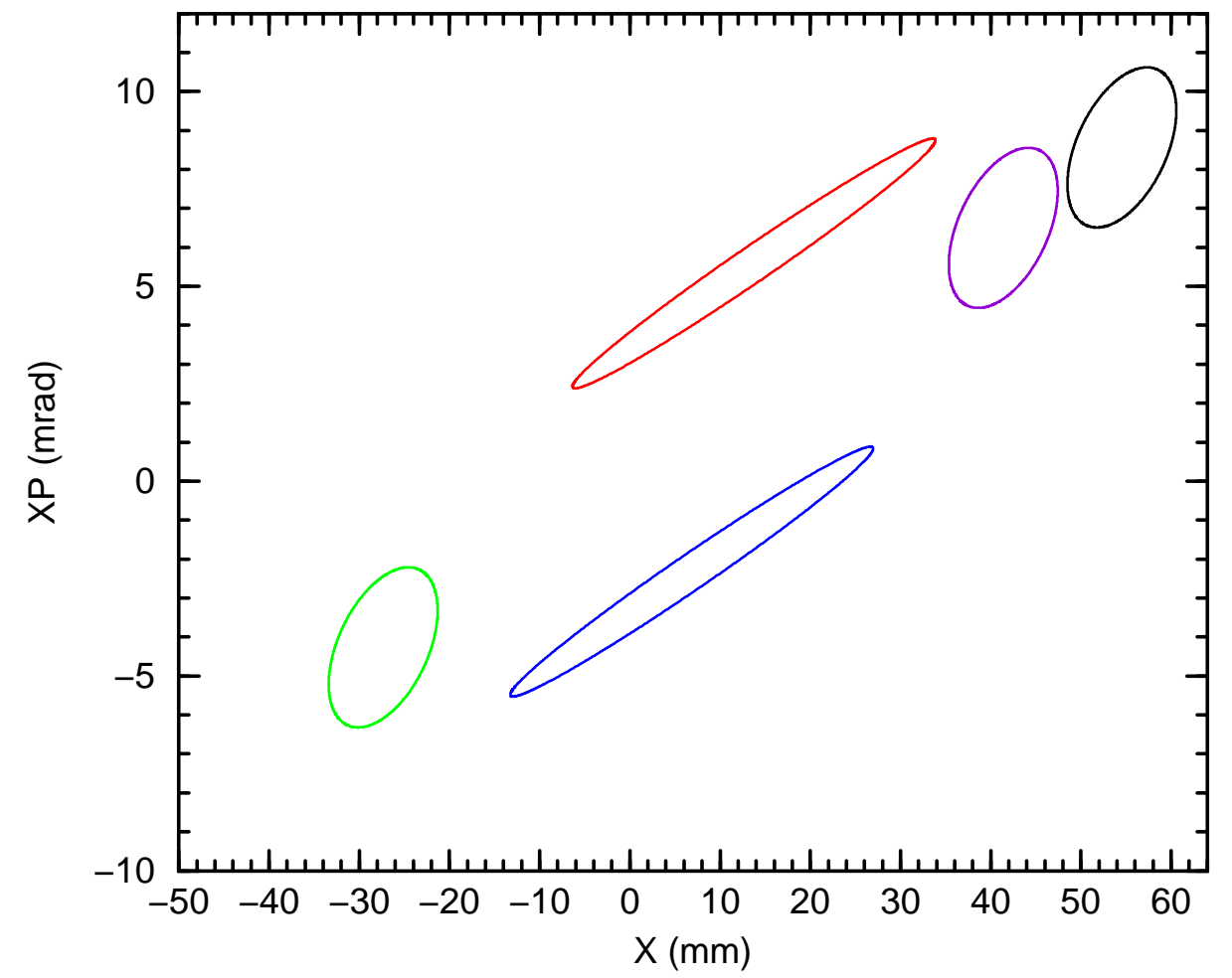

Figure 13: The black, red, green, blue, and violet curves are the beam ellipse after $0,1,2,3$, and 4 turns, respectively, from launch time 6.0 turns. As before, the initial (black) ellipse has parameter $B=0.3$ and area $\pi \epsilon_{0}=$ $11.0 \pi \mathrm{mm}$ milliradians. The outer side of the $1 \mathrm{~mm}$ thick inflector septum is located at $X=48.5 \mathrm{~mm}$. The tunes are $Q_{H}=4.75$ and $Q_{V}=4.81$. 


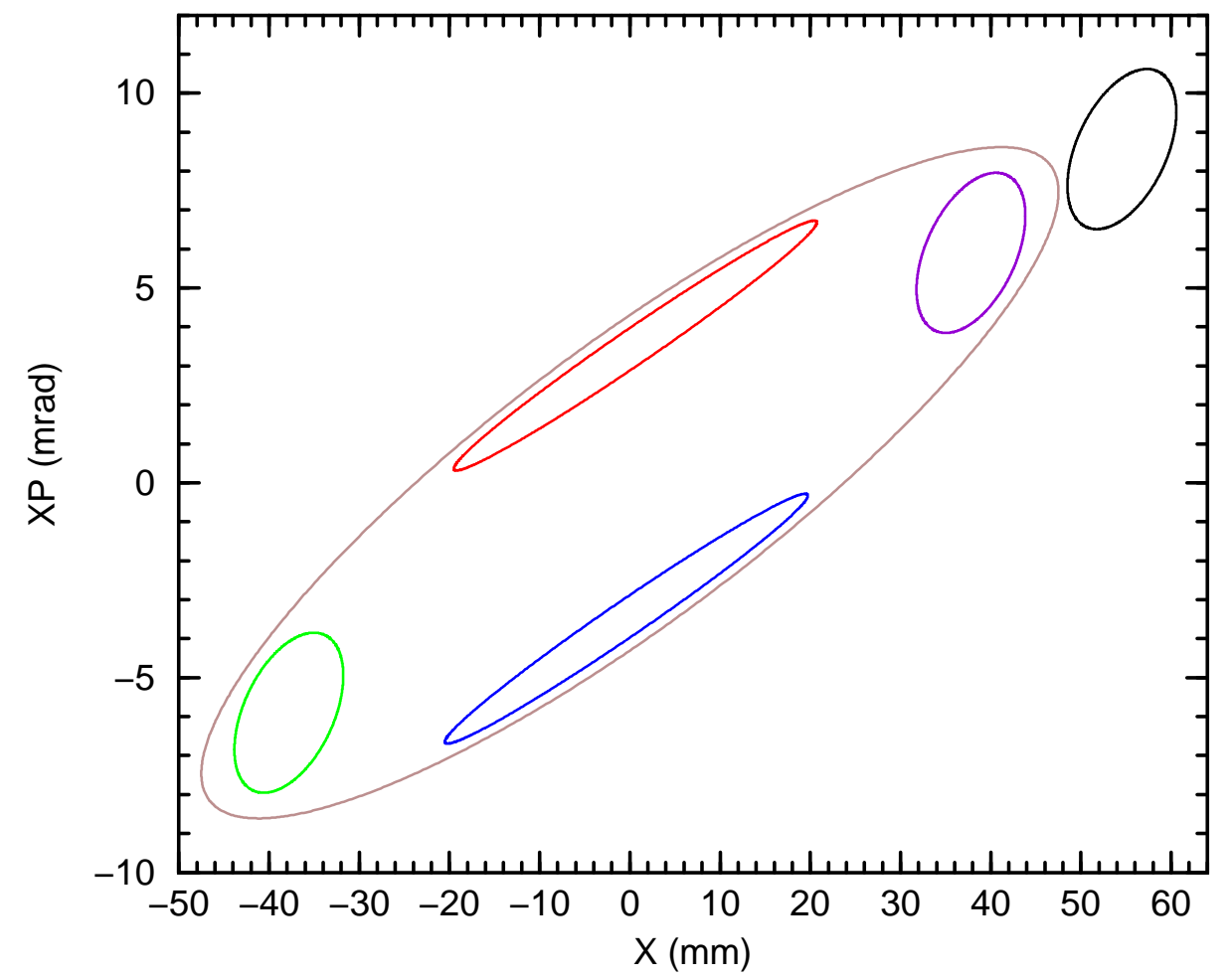

Figure 14: The black, red, green, blue, and violet curves are the beam ellipse after $0,5,6,7$, and 8 turns, respectively, from launch time 6.0 turns. The large brown ellipse is the machine acceptance, taken to be $204.5 \pi \mathrm{mm}$ milliradians. The initial (black) ellipse launched at time 6.0 turns therefore ends up inside but close to the edge of the machine acceptance after the bump has collapsed. 


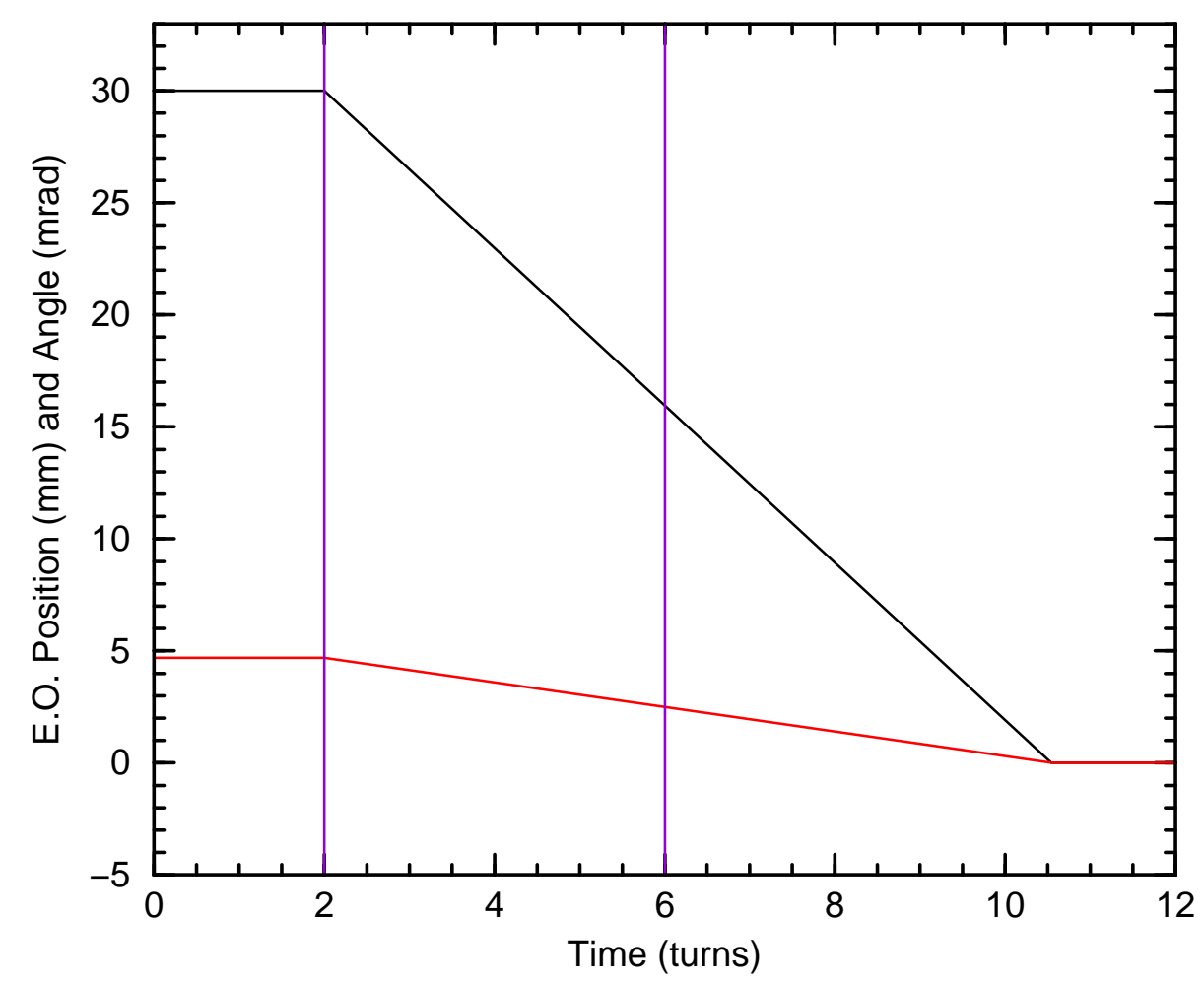

Figure 15: Here again the black and red curves are the injection bump position $X_{c}$ and angle $X_{c}^{\prime}=-\alpha_{I} X_{c} / \beta_{I}$ as functions of time for four-turn injection of EBIS beams. The time axis is marked in turns, where one turn is $10.276 \mu \mathrm{s}$. The beam pulse occurs between the two violet lines as before, but the horizontal and vertical tunes are now $Q_{H}=4.74$ and $Q_{V}=4.81$. The MAD program gives $\alpha_{I}=-1.732$ and $\beta_{I}=11.047 \mathrm{~m}$. The magnetic rigidity is taken to be $1.255 \mathrm{Tm}$ which is the expected maximum for EBIS beams. 


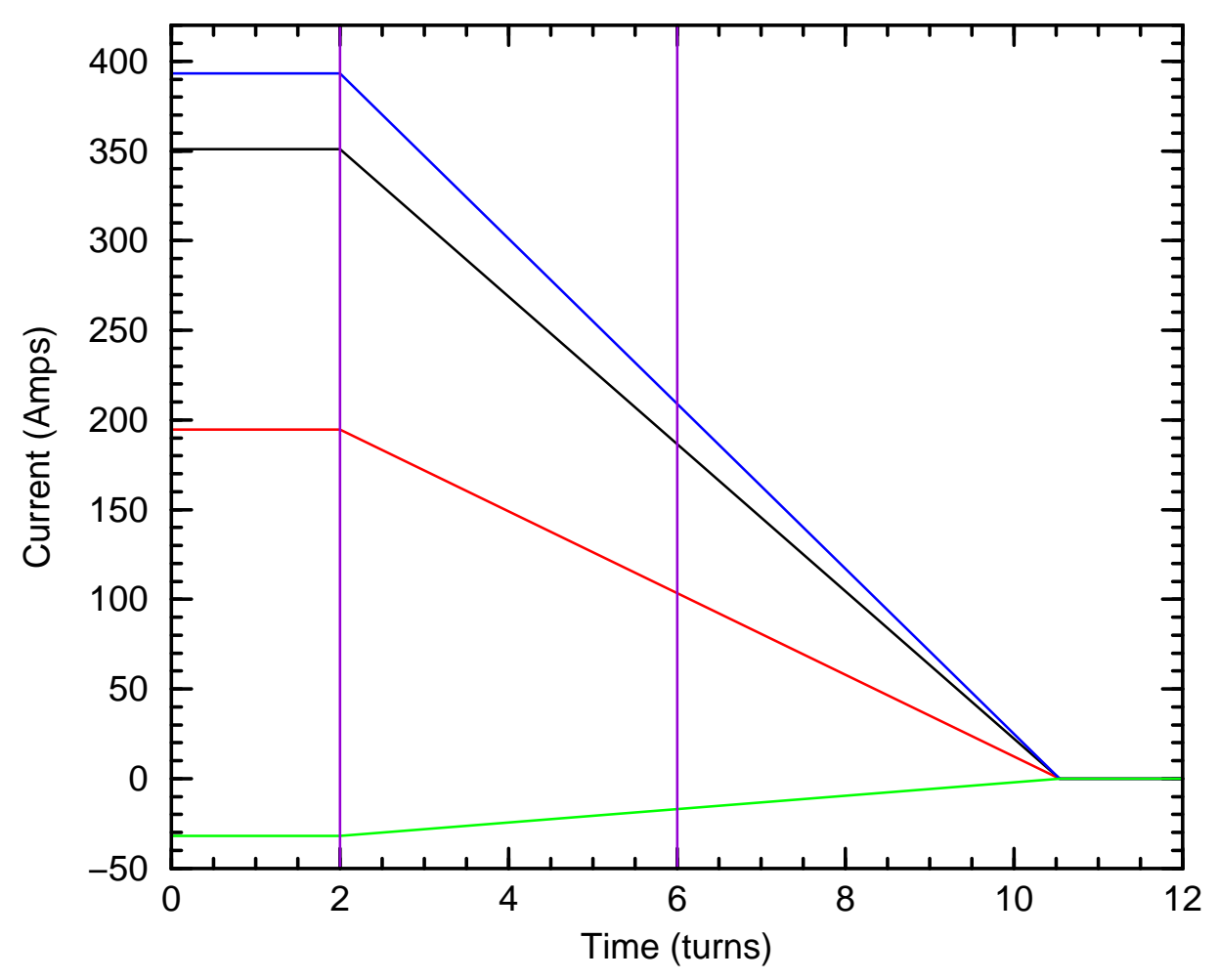

Figure 16: Here the black, red, blue, and green curves are the C1, C3, C7, and D1 dipole currents, respectively, for the injection bump function of Figure 15. The four-turn EBIS beam pulse occurs between the two violet lines. The rate of fall of the $\mathrm{C} 7$ dipole current (blue curve) is $4.47 \mathrm{~A} / \mu \mathrm{s}$. 


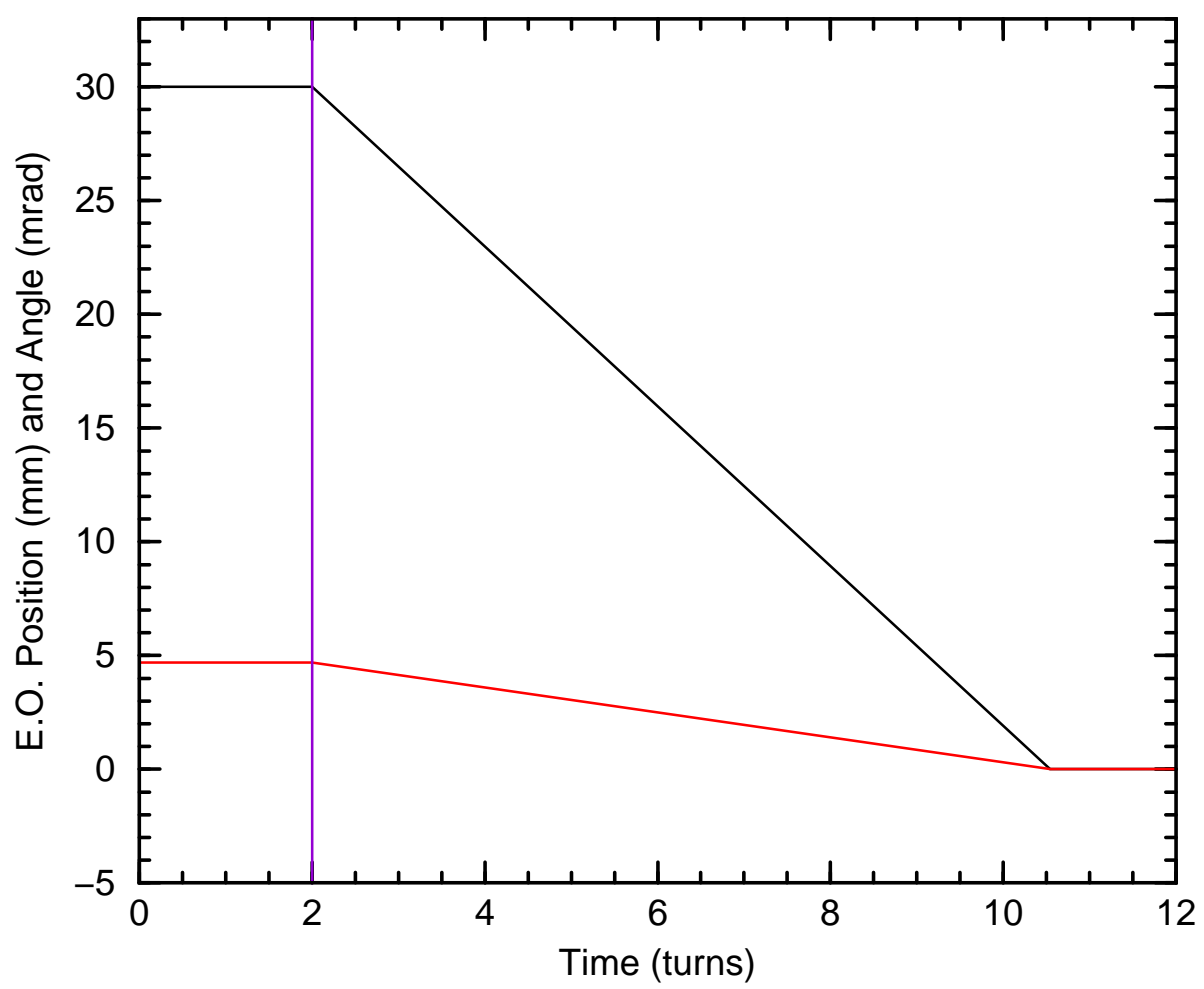

Figure 17: We consider the evolution of a beam ellipse launched from the injection point (i.e. the exit of the C3 inflector) at time 2.0 turns as indicated by the violet line above. Figures 18 through 21 show the evolution of the beam ellipse. The necessary computations were carried out with Fortran program "EBinject" using MAD Twiss file TwissQH4740000. 


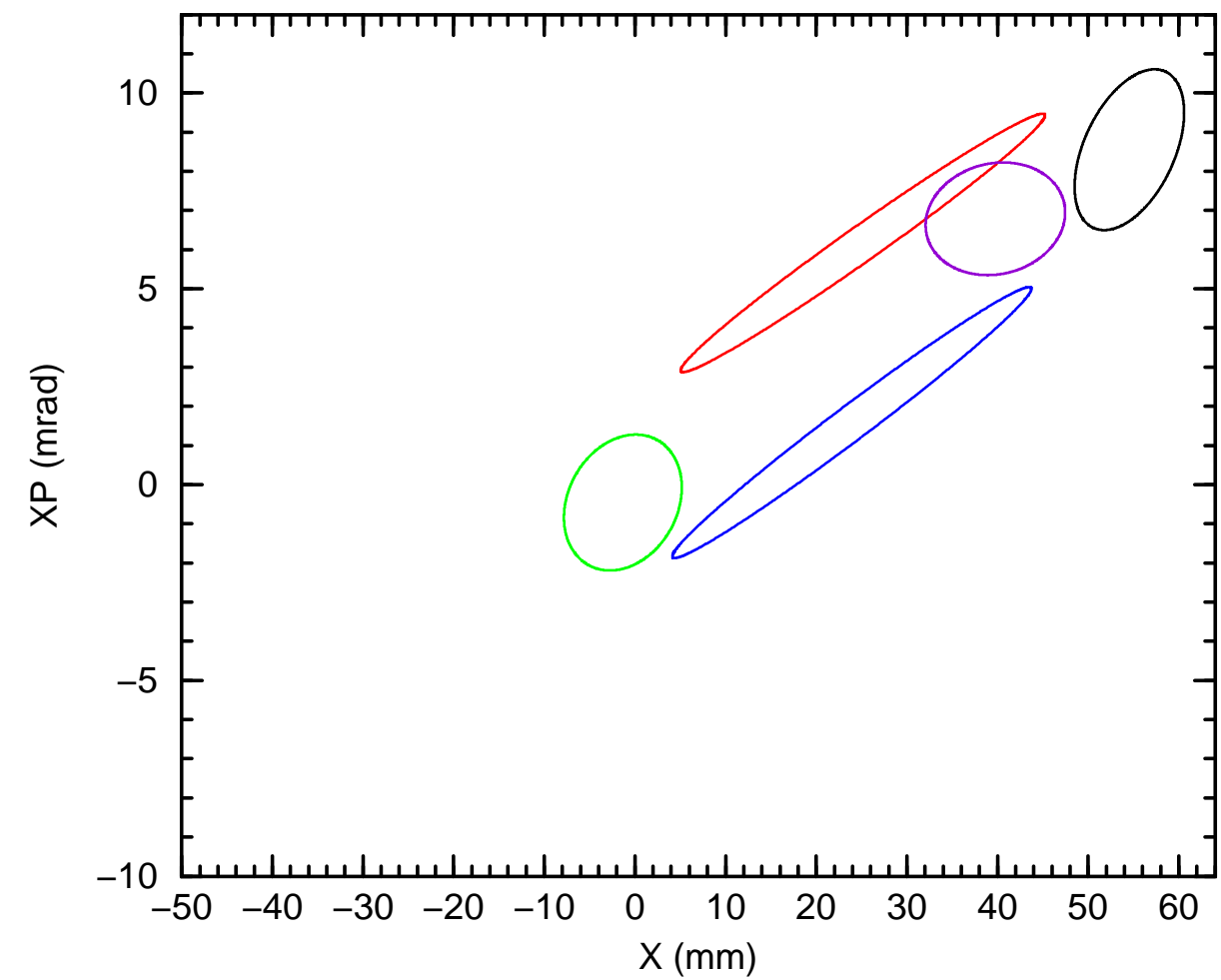

Figure 18: The black, red, green, blue, and violet curves are the beam ellipse after $0,1,2,3$, and 4 turns, respectively, from launch time 2.0 turns. The initial (black) ellipse has parameter $B=0.3$ and area $\pi \epsilon_{0}=11.0 \pi \mathrm{mm}$ milliradians. The outer side of the $1 \mathrm{~mm}$ thick inflector septum is located at $X=48.5 \mathrm{~mm}$. The tunes are $Q_{H}=4.74$ and $Q_{V}=4.81$. 


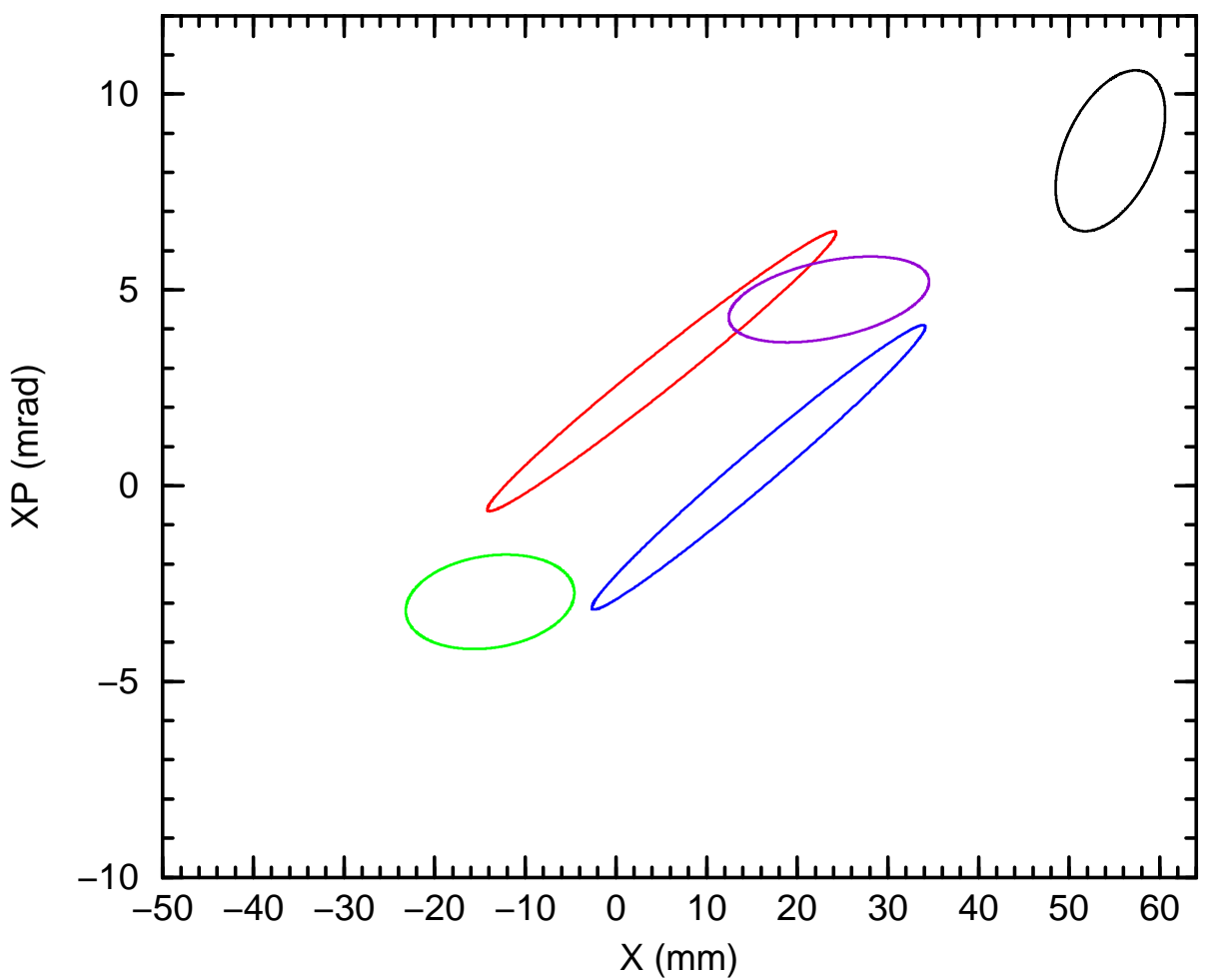

Figure 19: The black, red, green, blue, and violet curves are the beam ellipse after $0,5,6,7$, and 8 turns, respectively, from launch time 2.0 turns. Note again that the injected ellipse moves with the falling bump. 


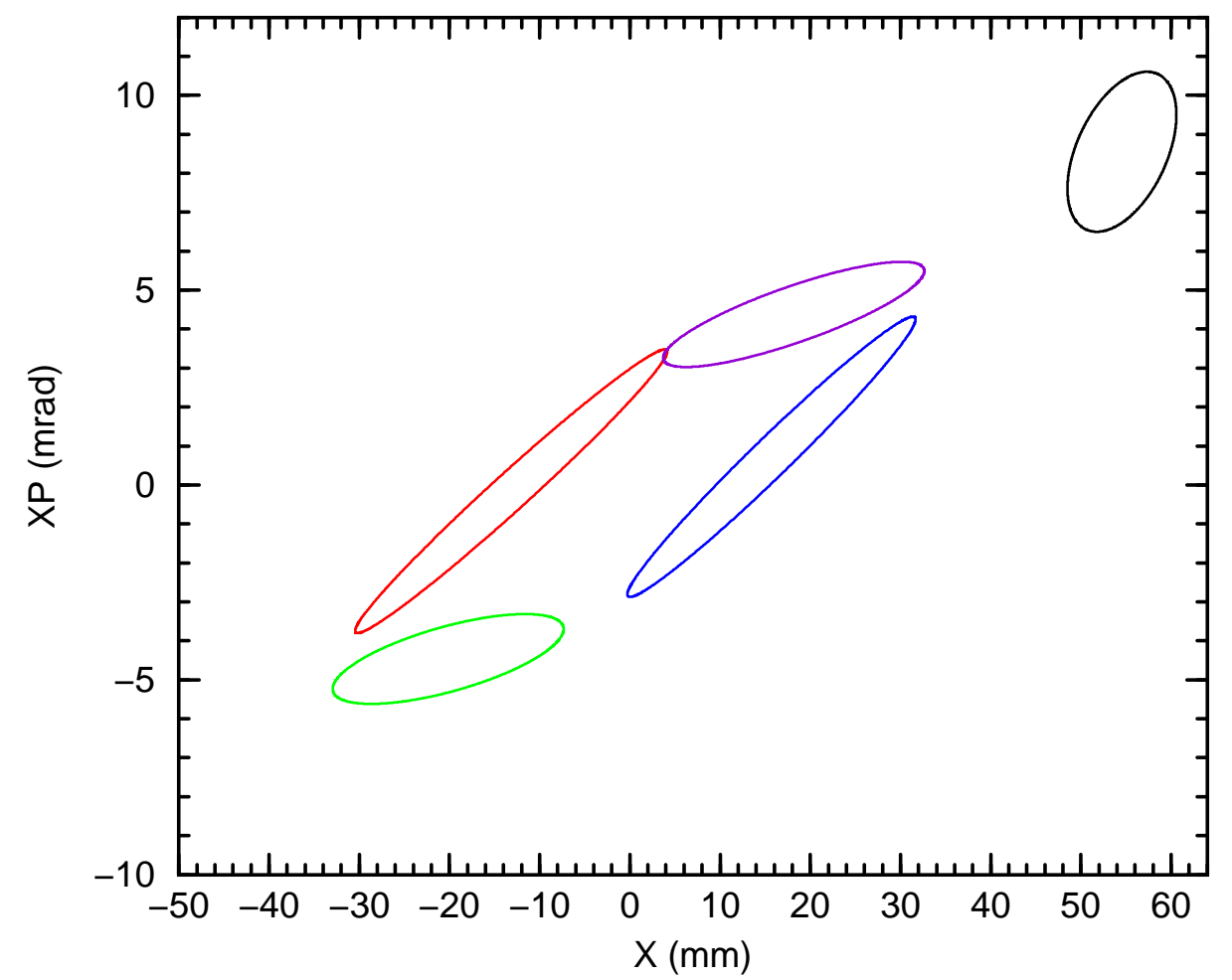

Figure 20: The black, red, green, blue, and violet curves are the beam ellipse after $0,9,10,11$, and 12 turns, respectively, from launch time 2.0 turns. 


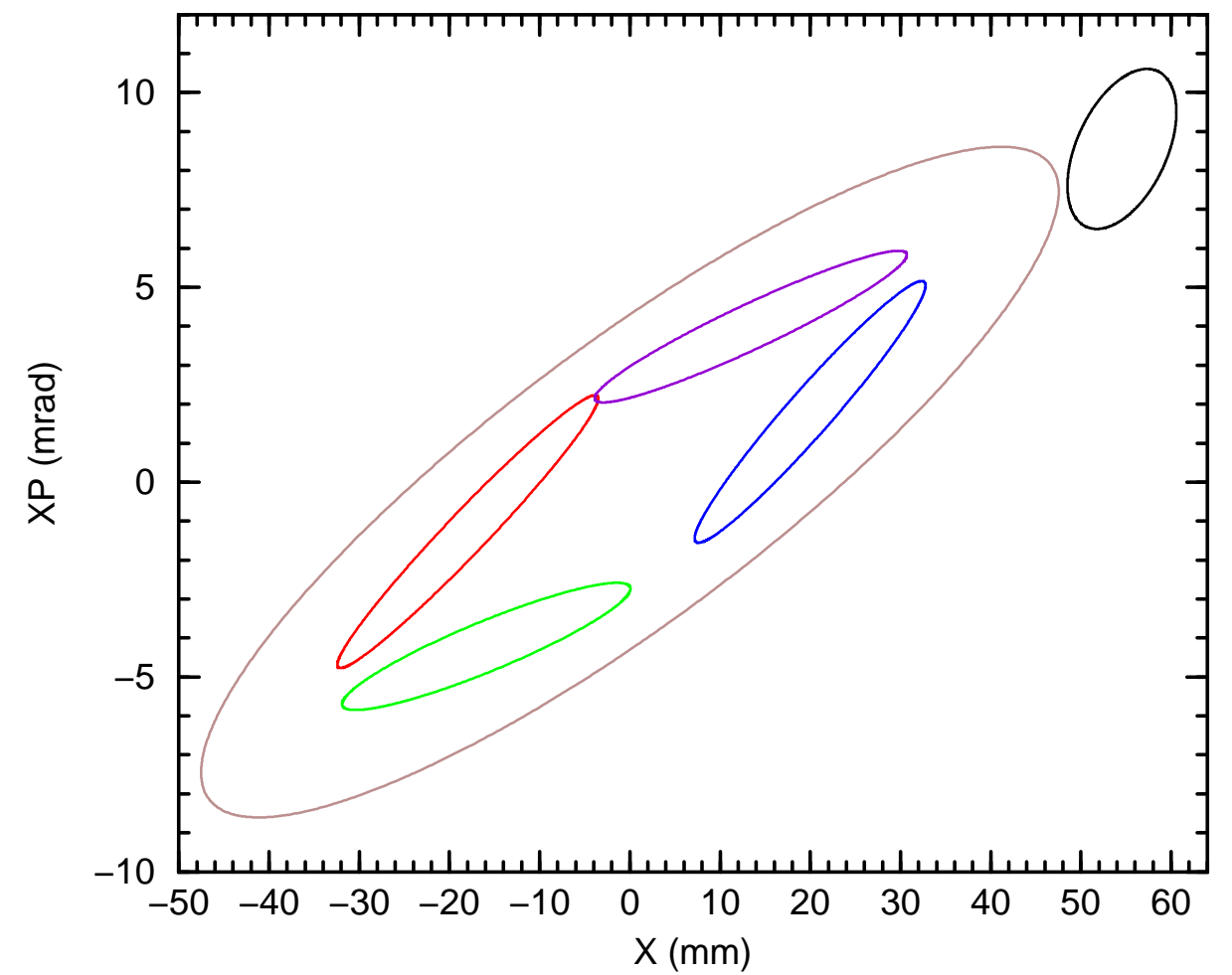

Figure 21: The black, red, green, blue, and violet curves are the beam ellipse after $0,13,14,15$, and 16 turns, respectively, from launch time 2.0 turns. The large brown ellipse is the machine acceptance, taken to be $204.5 \pi \mathrm{mm}$ milliradians. The initial (black) ellipse launched at time 2.0 turns therefore ends up well inside the machine acceptance after the bump has collapsed. 


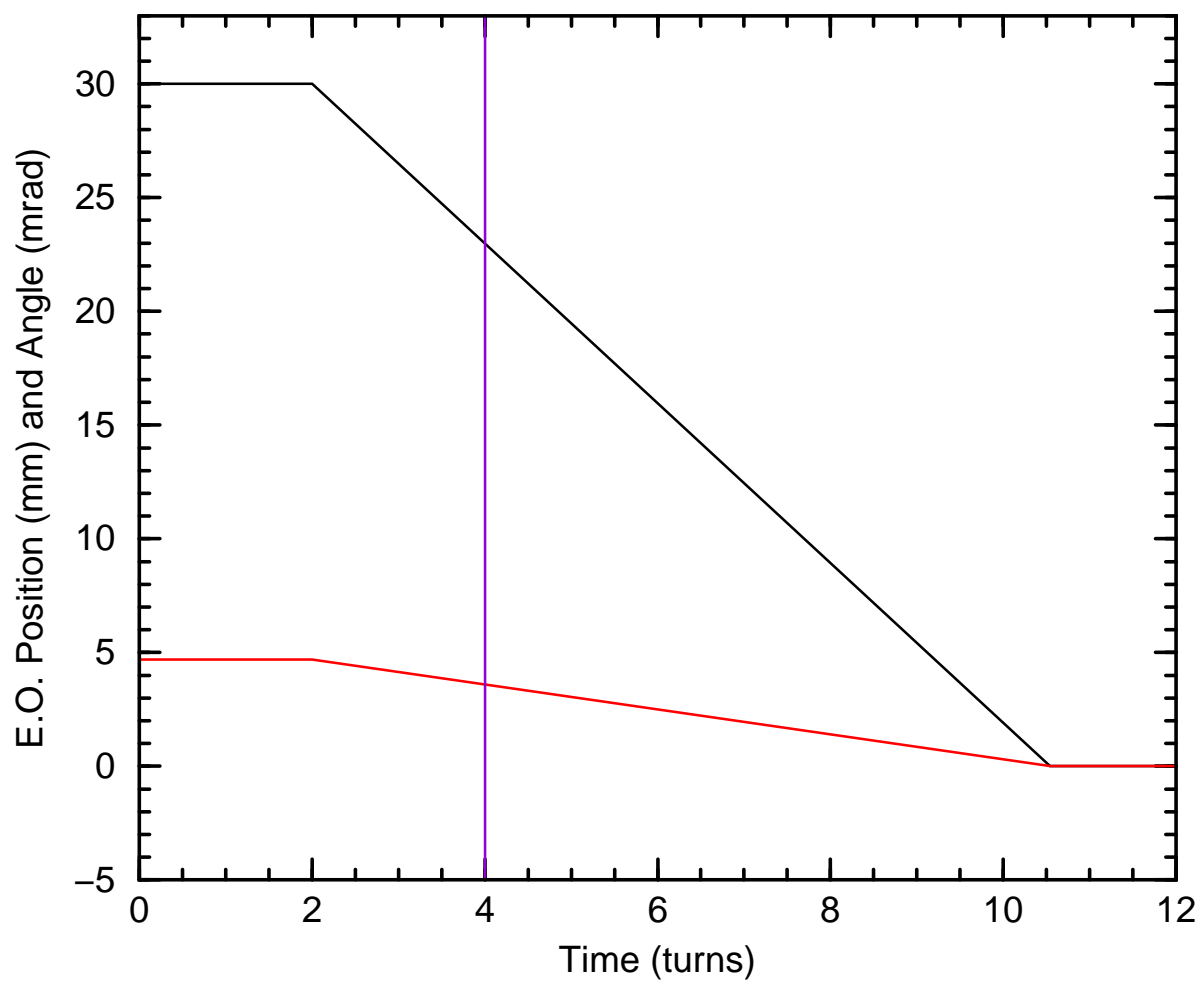

Figure 22: Now consider the evolution of a beam ellipse launched from the injection point at time 4.0 turns as indicated by the violet line above. Figures $\mathbf{2 3}$ through $\mathbf{2 6}$ show the evolution of the beam ellipse. As before, the necessary computations were carried out with Fortran program "EBinject" using MAD Twiss file TwissQH4740000. 


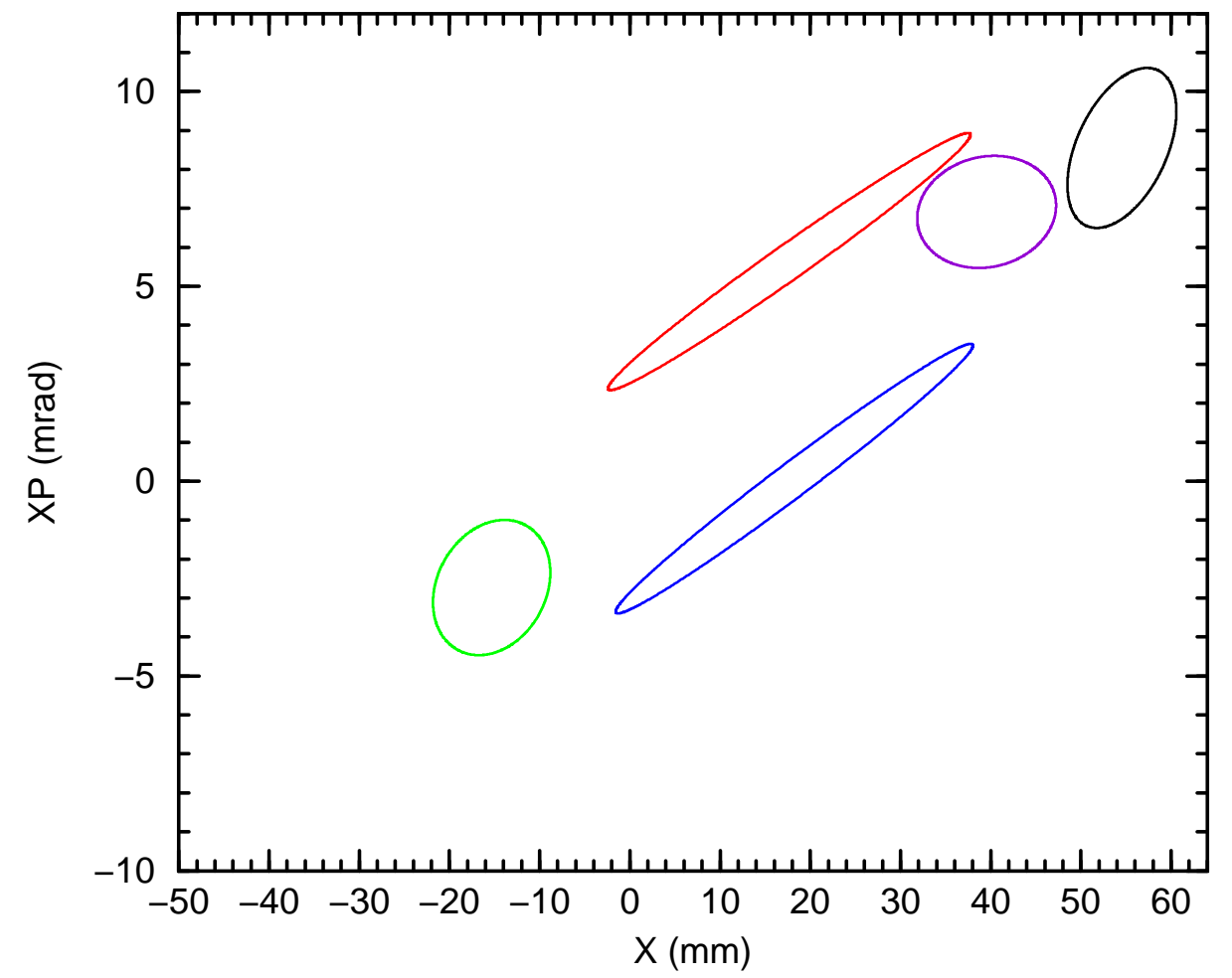

Figure 23: The black, red, green, blue, and violet curves are the beam ellipse after $0,1,2,3$, and 4 turns, respectively, from launch time 4.0 turns. As before, the initial (black) ellipse has parameter $B=0.3$ and area $\pi \epsilon_{0}=$ $11.0 \pi \mathrm{mm}$ milliradians. The outer side of the $1 \mathrm{~mm}$ thick inflector septum is located at $X=48.5 \mathrm{~mm}$. The tunes are $Q_{H}=4.74$ and $Q_{V}=4.81$. 


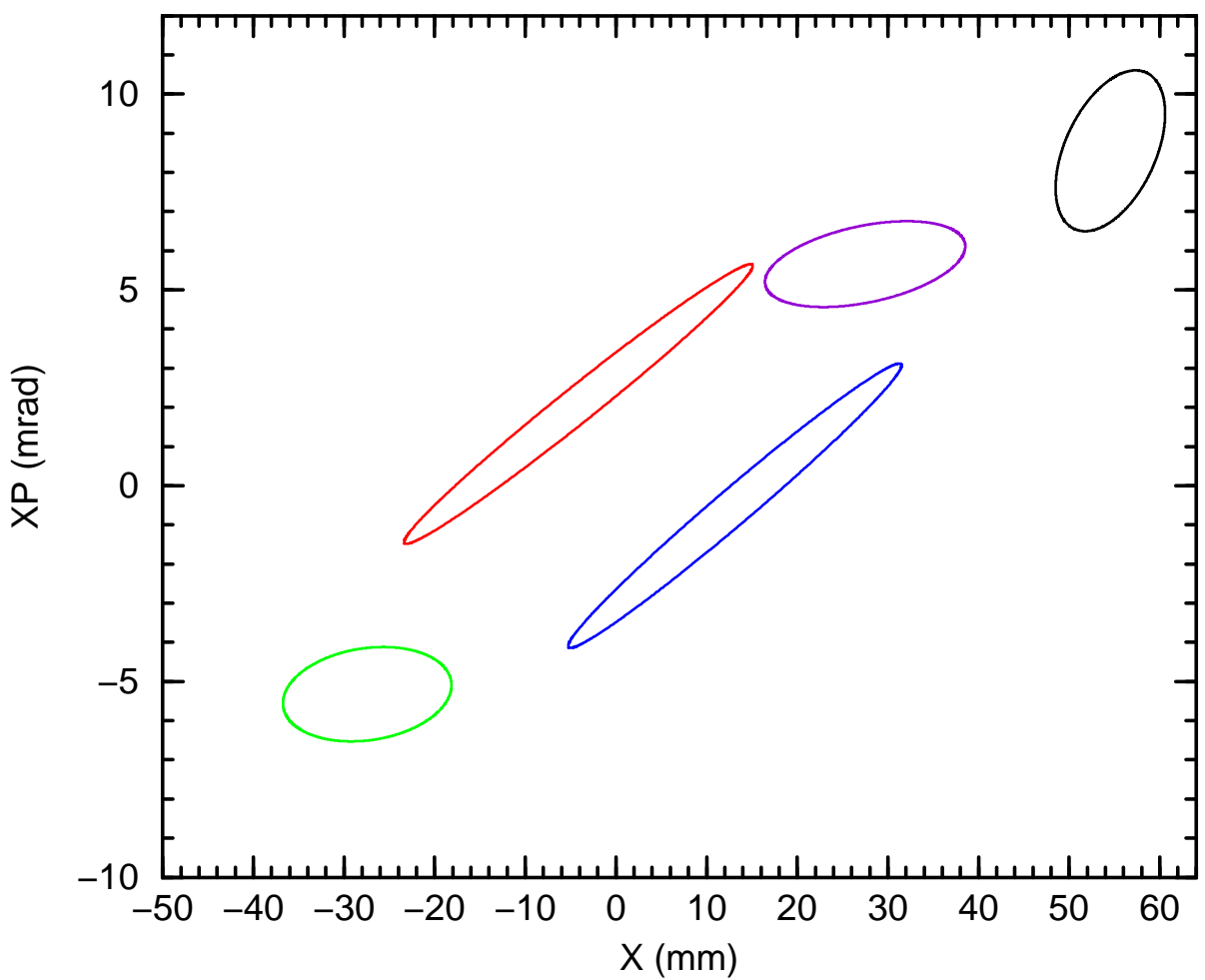

Figure 24: The black, red, green, blue, and violet curves are the beam ellipse after $0,5,6,7$, and 8 turns, respectively, from launch time 4.0 turns. Note again that the injected ellipse moves with the falling bump. 


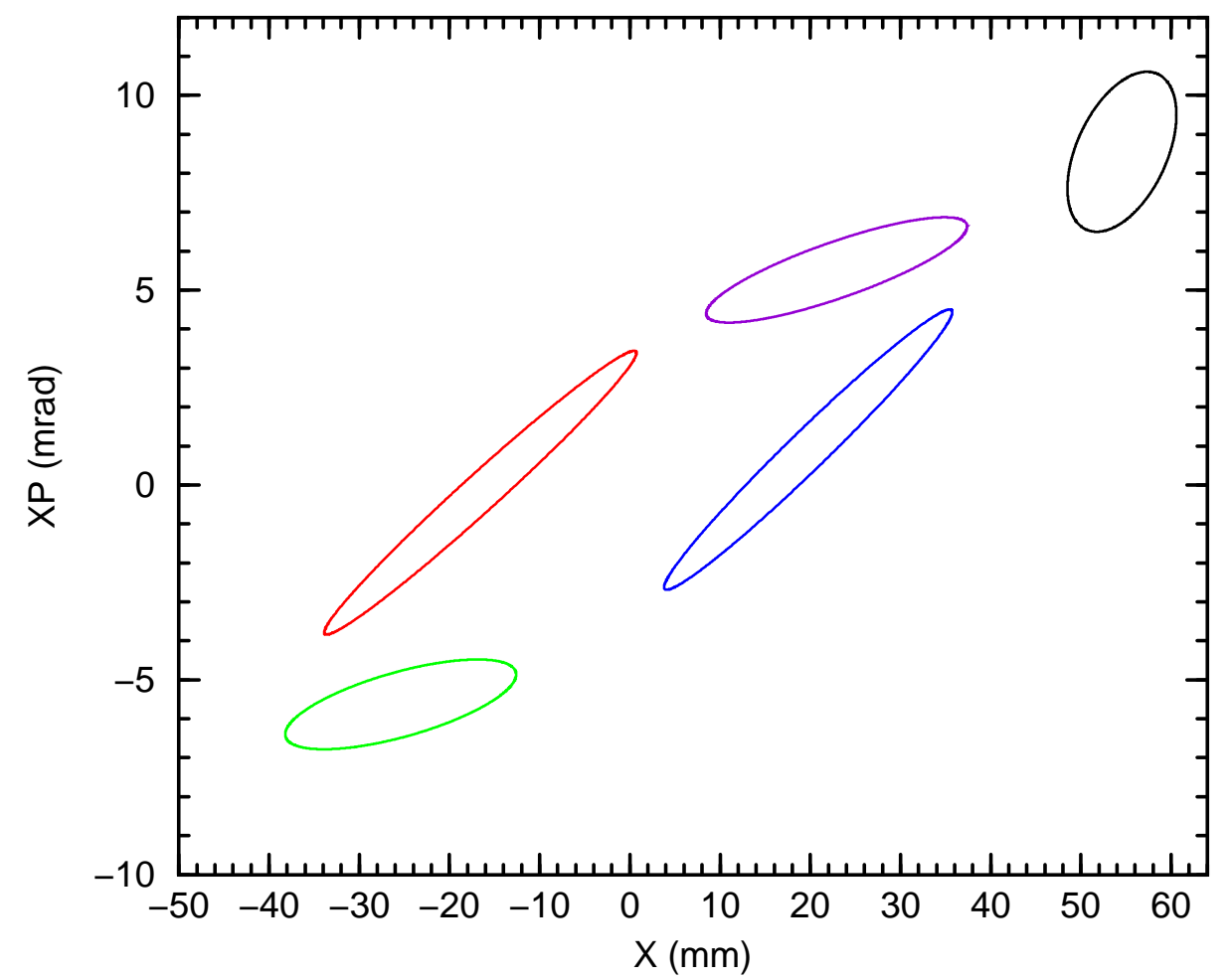

Figure 25: The black, red, green, blue, and violet curves are the beam ellipse after $0,9,10,11$, and 12 turns, respectively, from launch time 4.0 turns. 


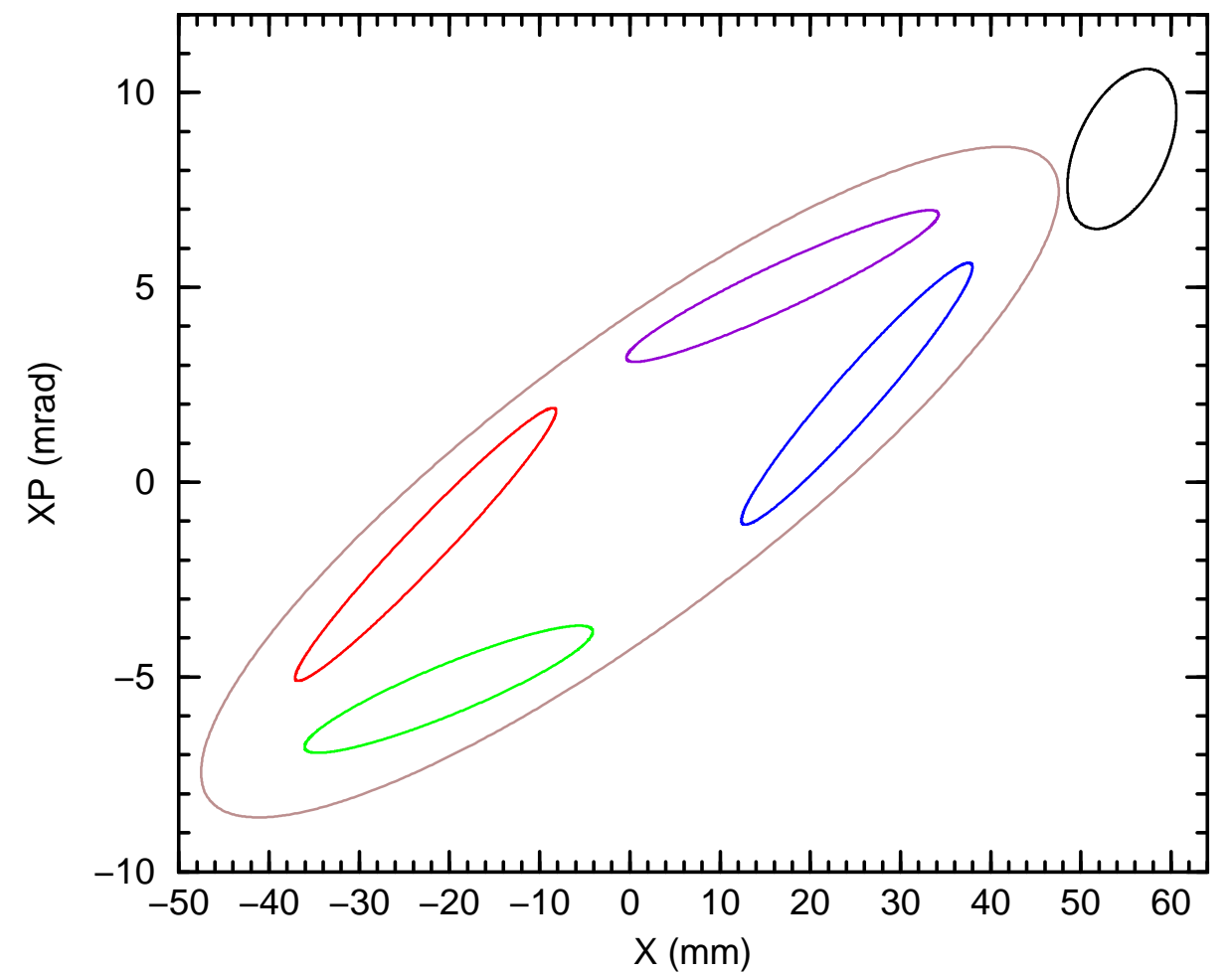

Figure 26: The black, red, green, blue, and violet curves are the beam ellipse after $0,13,14,15$, and 16 turns, respectively, from launch time 4.0 turns. The large brown ellipse is the machine acceptance, taken to be $204.5 \pi \mathrm{mm}$ milliradians. The initial (black) ellipse launched at time 4.0 turns therefore ends up well inside the machine acceptance after the bump has collapsed. 


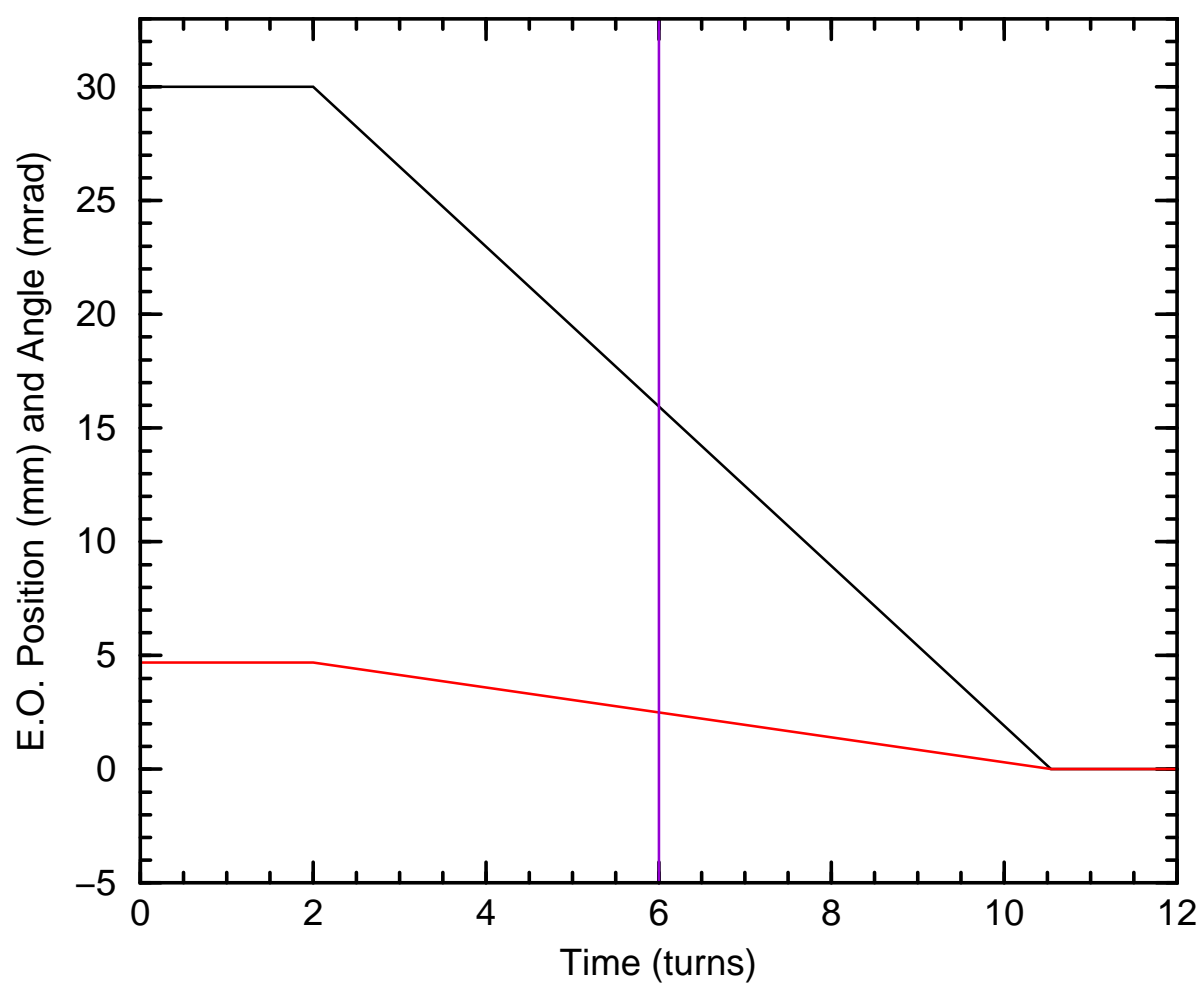

Figure 27: Now consider the evolution of a beam ellipse launched from the injection point at time 6.0 turns as indicated by the violet line above. Figures 28 and 29 show the evolution of the beam ellipse. As before, the necessary computations were carried out with Fortran program "EBinject" using MAD Twiss file TwissQH4740000. 


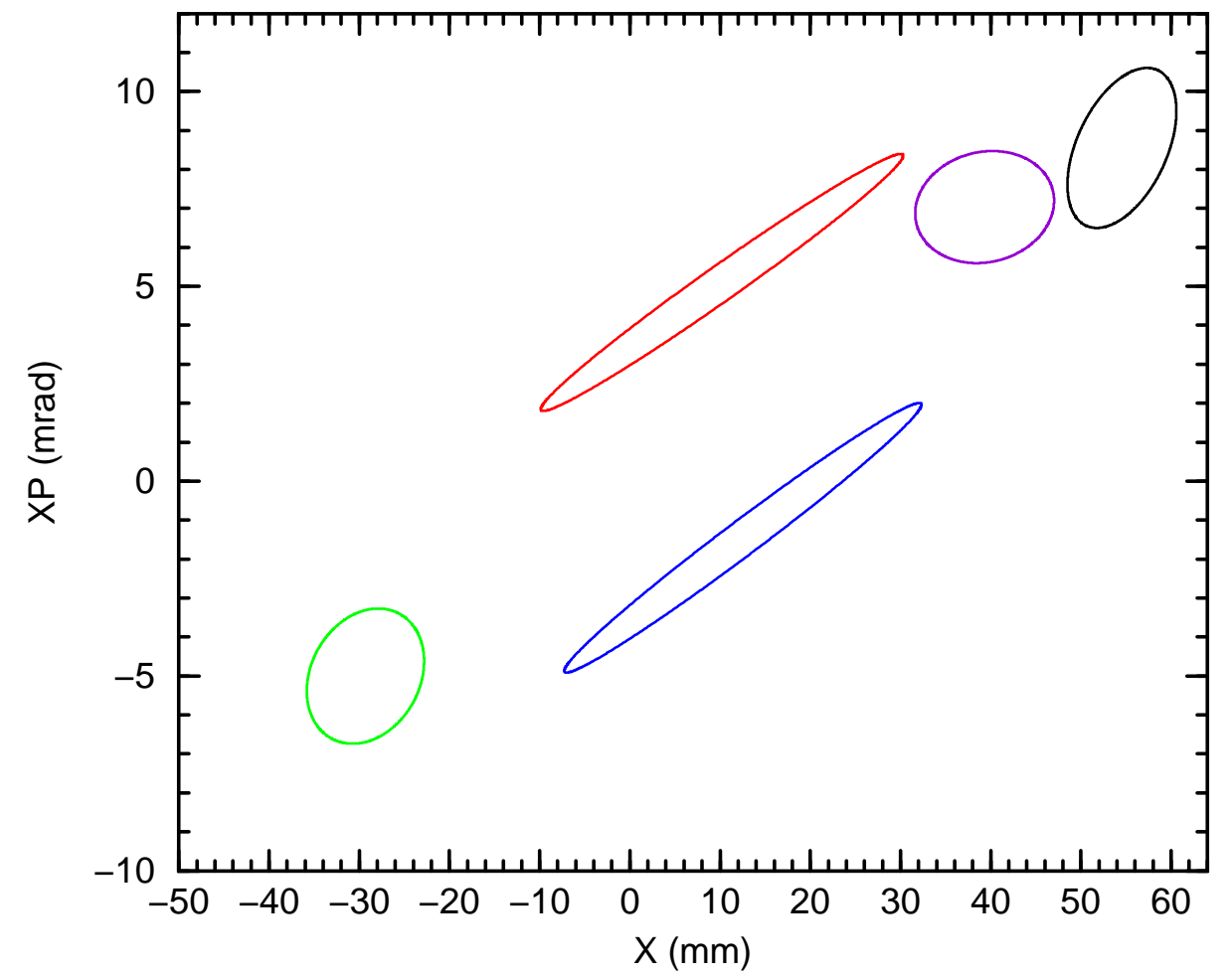

Figure 28: The black, red, green, blue, and violet curves are the beam ellipse after $0,1,2,3$, and 4 turns, respectively, from launch time 6.0 turns. As before, the initial (black) ellipse has parameter $B=0.3$ and area $\pi \epsilon_{0}=$ $11.0 \pi \mathrm{mm}$ milliradians. The outer side of the $1 \mathrm{~mm}$ thick inflector septum is located at $X=48.5 \mathrm{~mm}$. The tunes are $Q_{H}=4.74$ and $Q_{V}=4.81$. 


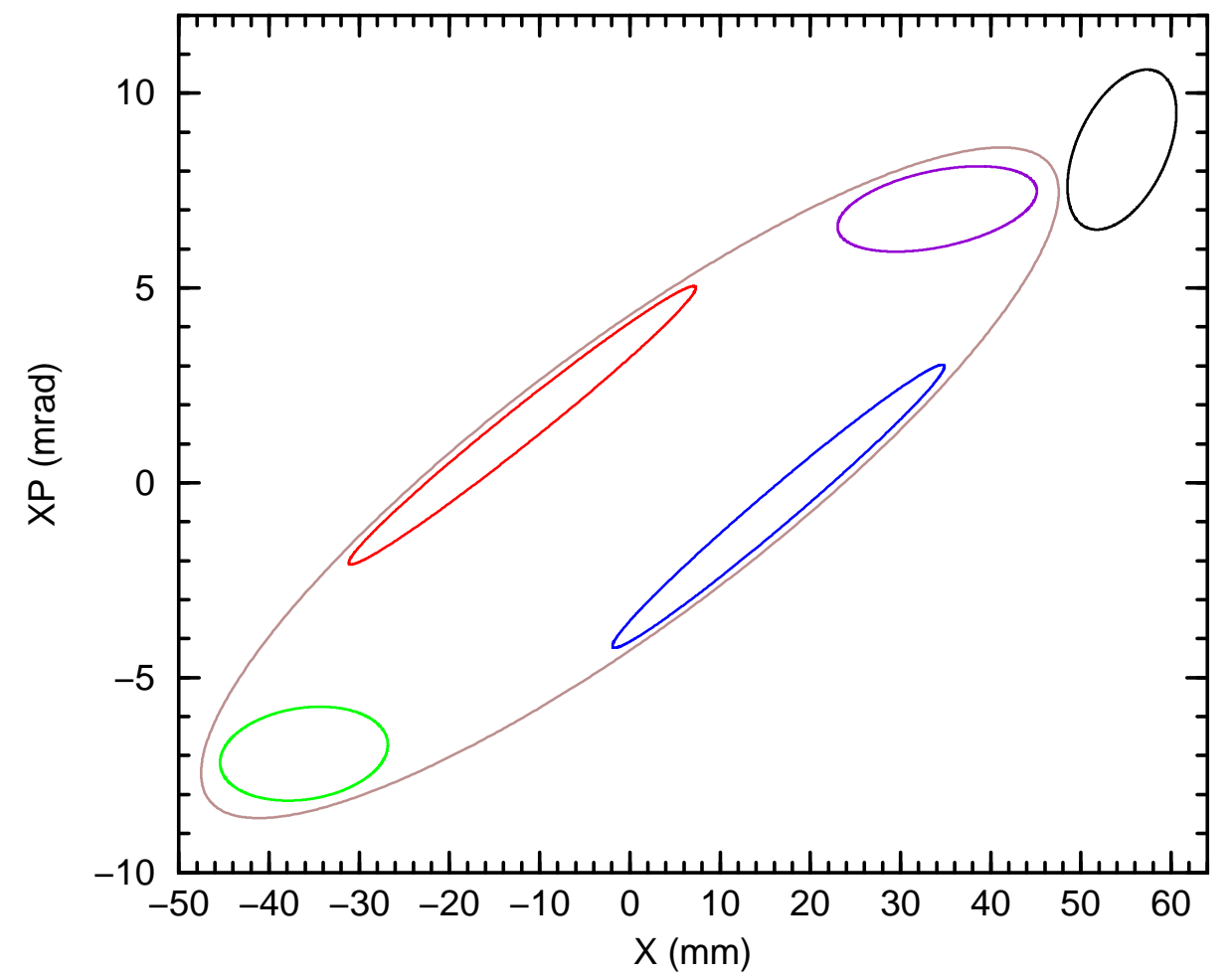

Figure 29: The black, red, green, blue, and violet curves are the beam ellipse after $0,5,6,7$, and 8 turns, respectively, from launch time 6.0 turns. The large brown ellipse is the machine acceptance, taken to be $204.5 \pi \mathrm{mm}$ milliradians. The initial (black) ellipse launched at time 6.0 turns therefore ends up inside but close to the edge of the machine acceptance after the bump has collapsed. 


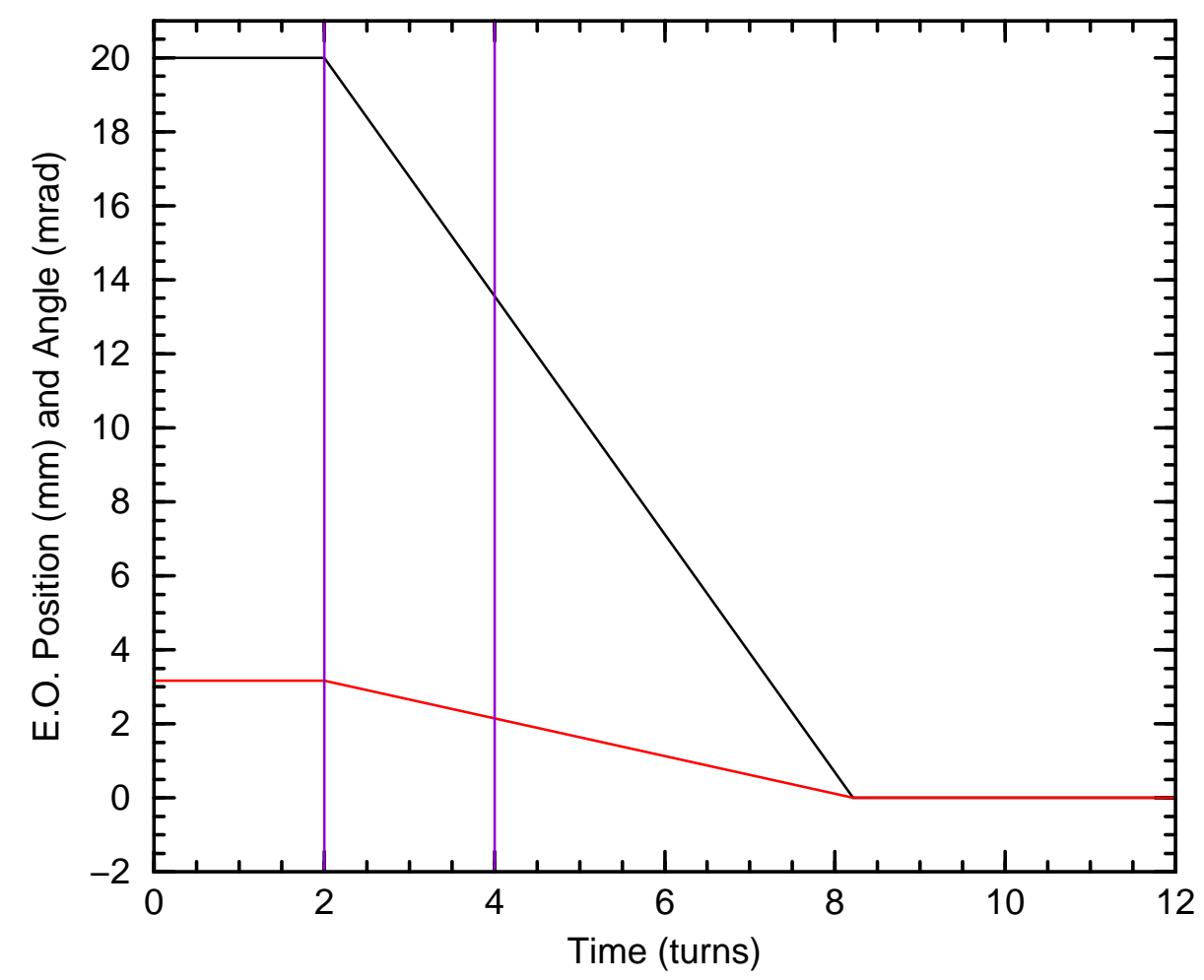

Figure 30: The black and red curves are the injection bump position $X_{c}$ and angle $X_{c}^{\prime}=-\alpha_{I} X_{c} / \beta_{I}$ as functions of time for two-turn injection of EBIS beams. The time axis is marked in turns, where one turn is $10.276 \mu \mathrm{s}$. The beam pulse occurs between the two violet lines. The horizontal and vertical tunes are now $Q_{H}=4.80$ and $Q_{V}=4.81$. The MAD program gives $\alpha_{I}=-1.737$ and $\beta_{I}=10.969 \mathrm{~m}$. The magnetic rigidity is taken to be 1.255 Tm which is the expected maximum for EBIS beams. 


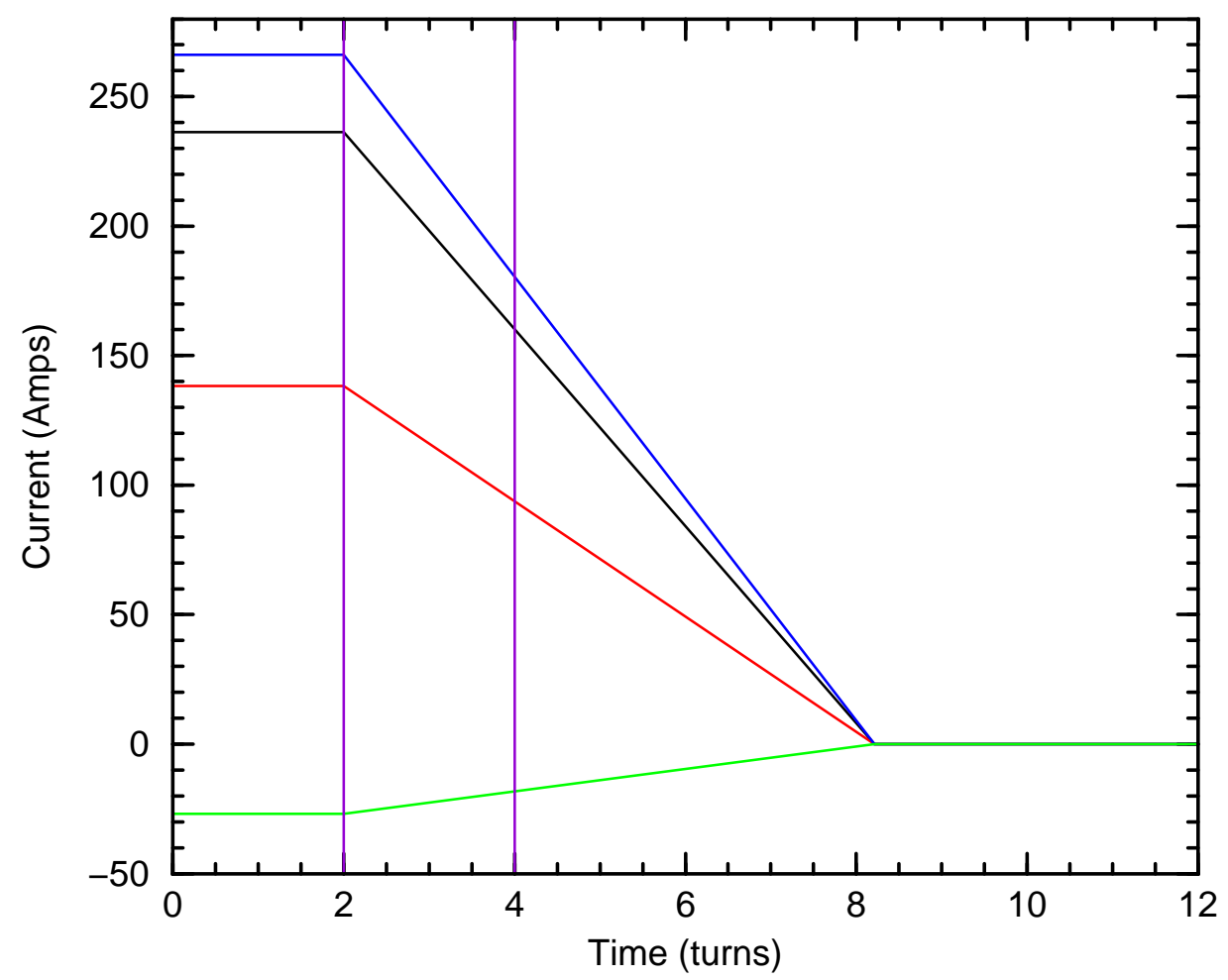

Figure 31: The black, red, blue, and green curves are the C1, C3, C7, and D1 dipole currents, respectively, for the injection bump function of Figure 30. The two-turn EBIS beam pulse occurs between the two violet lines. The rate of fall of the $\mathrm{C} 7$ dipole current (blue curve) is $4.16 \mathrm{~A} / \mu \mathrm{s}$. 


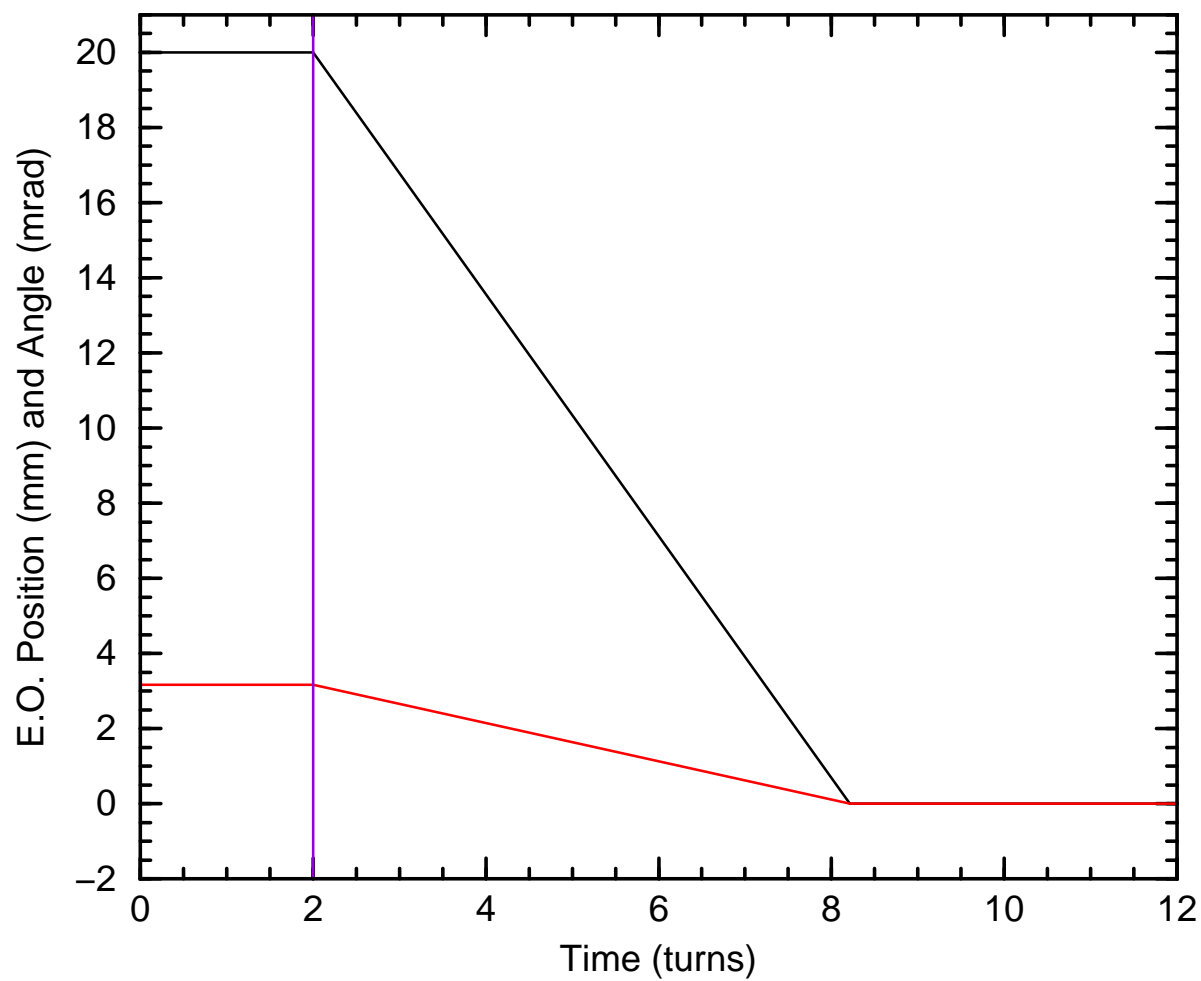

Figure 32: We consider the evolution of a beam ellipse launched from the injection point (i.e. the exit of the $\mathrm{C} 3$ inflector) at time 2.0 turns as indicated by the violet line above. Figures $\mathbf{3 3}$ and $\mathbf{3 4}$ show the evolution of the beam ellipse. The necessary computations were carried out with Fortran program "EBinject" using MAD Twiss file TwissEBinjct80. 


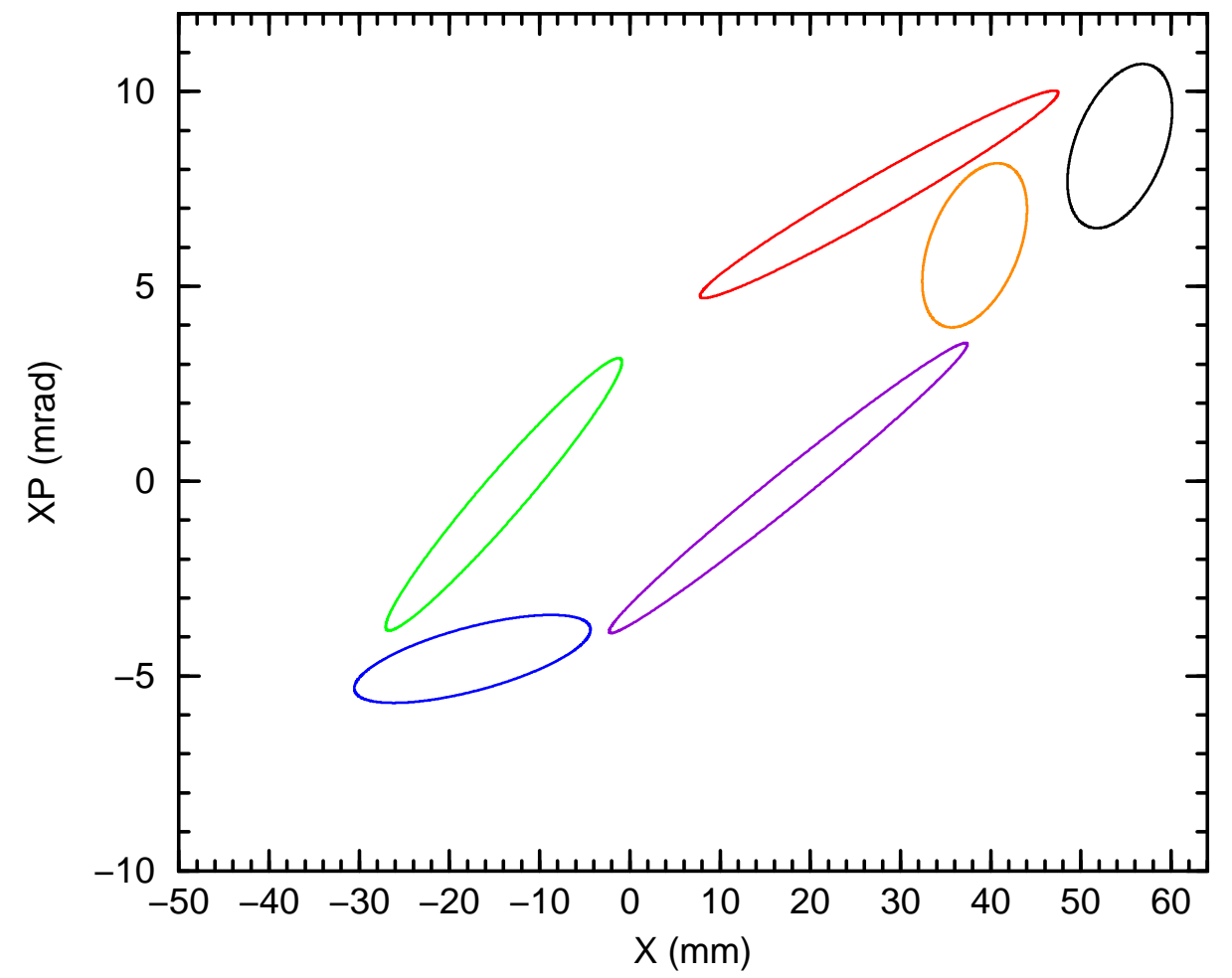

Figure 33: The black, red, green, blue, violet, and orange curves are beam ellipse after $0,1,2,3,4$, and 5 turns, respectively, from launch time 2.0 turns. The initial (black) ellipse has parameter $B=0.2793$ and area $\pi \epsilon_{0}=11.0 \pi$ $\mathrm{mm}$ milliradians. The outer side of the $1 \mathrm{~mm}$ thick inflector septum is located at $X=48.5 \mathrm{~mm}$. The tunes are $Q_{H}=4.800$ and $Q_{V}=4.810$. 


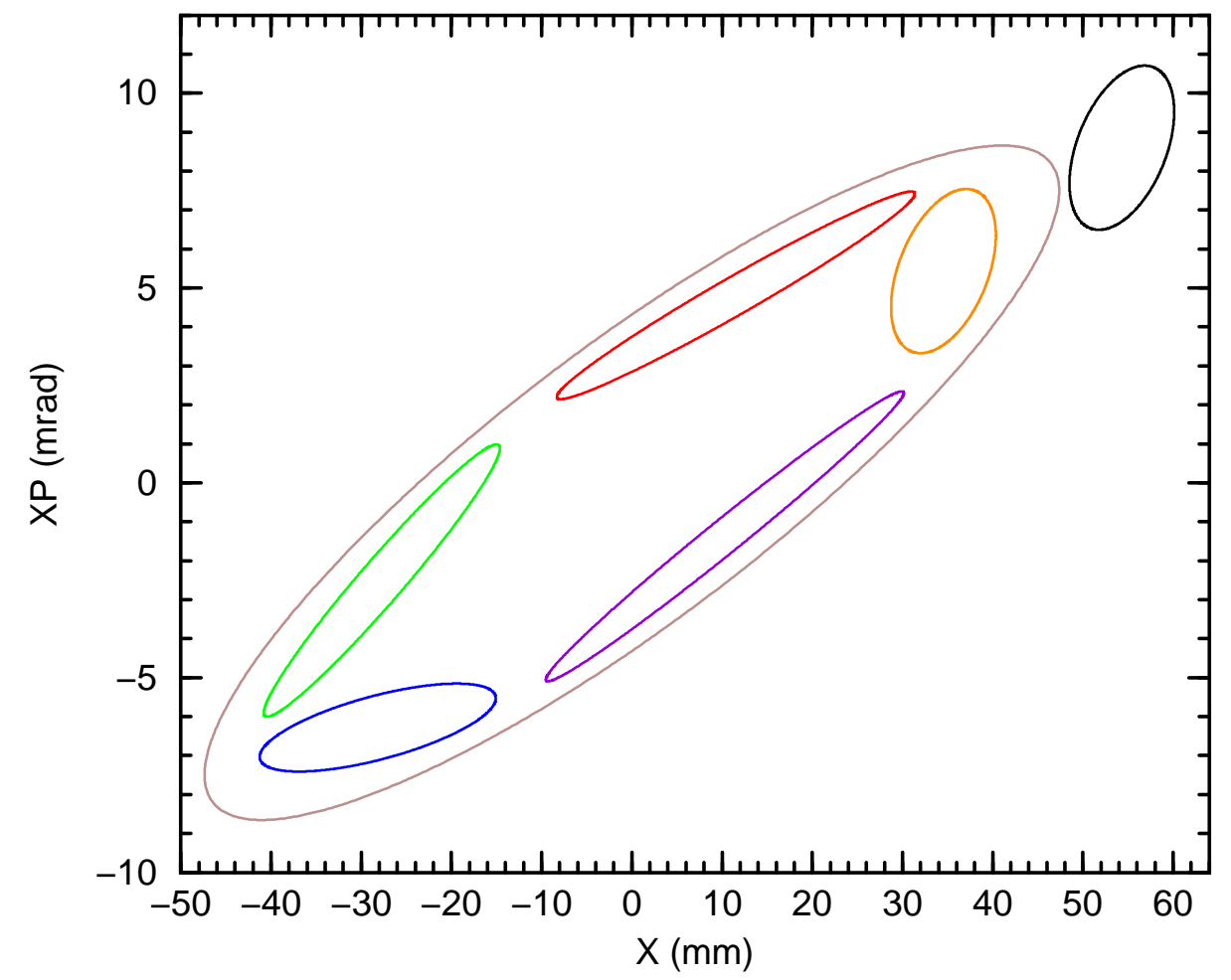

Figure 34: The black, red, green, blue, violet, and orange curves are the beam ellipse after $0,6,7,8,9$, and 10 turns, respectively, from launch time 2.0 turns. The large brown ellipse is the machine acceptance, taken to be $204.5 \pi \mathrm{mm}$ milliradians. The initial (black) ellipse launched at time 2.0 turns therefore ends up well inside the machine acceptance after the bump has collapsed. 


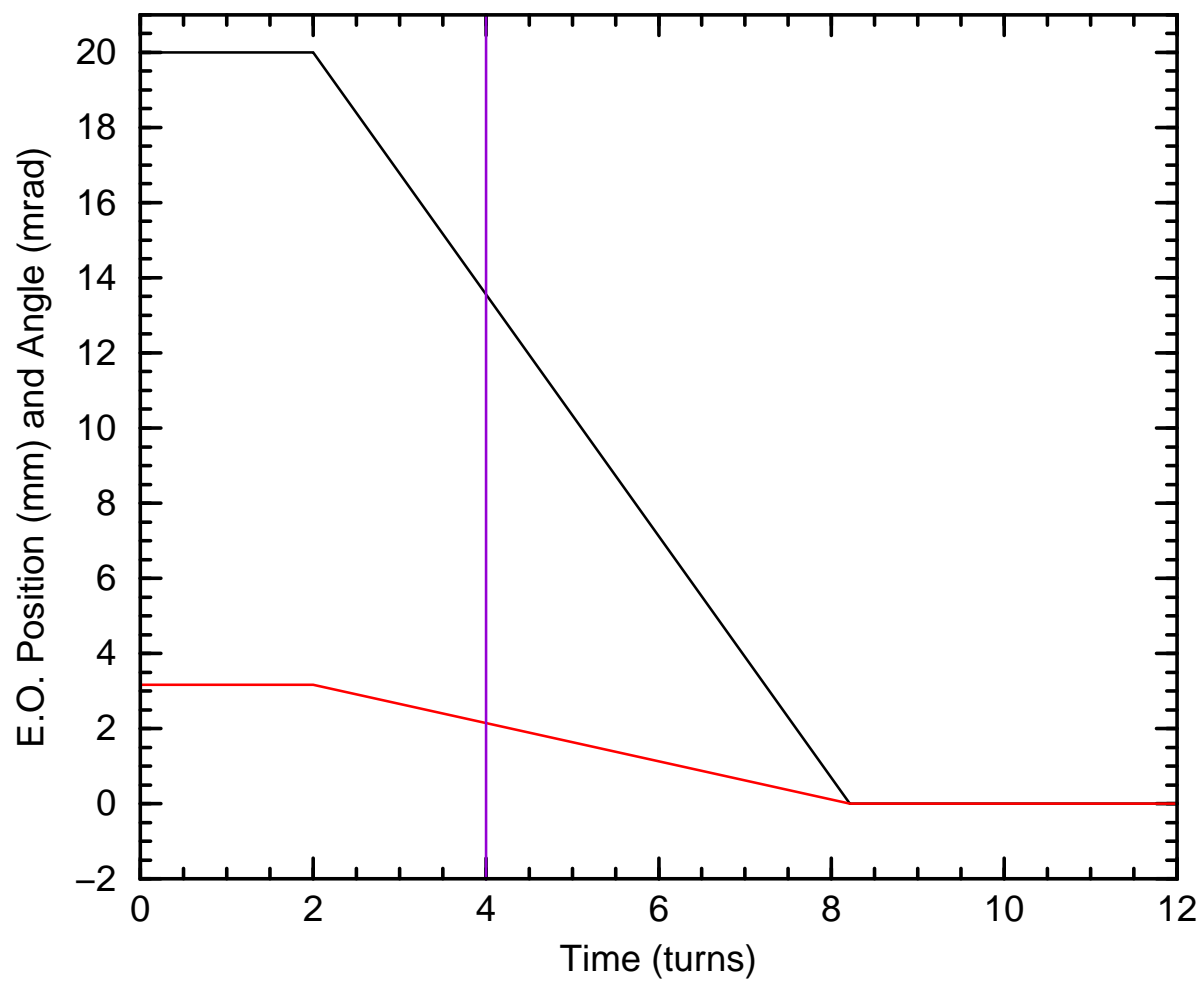

Figure 35: Now consider the evolution of a beam ellipse launched from the injection point at time 4.0 turns as indicated by the violet line above. Figures 36 and $\mathbf{3 7}$ show the evolution of the beam ellipse. As before, the necessary computations were carried out with Fortran program "EBinject" using MAD Twiss file TwissEBinjct80. 


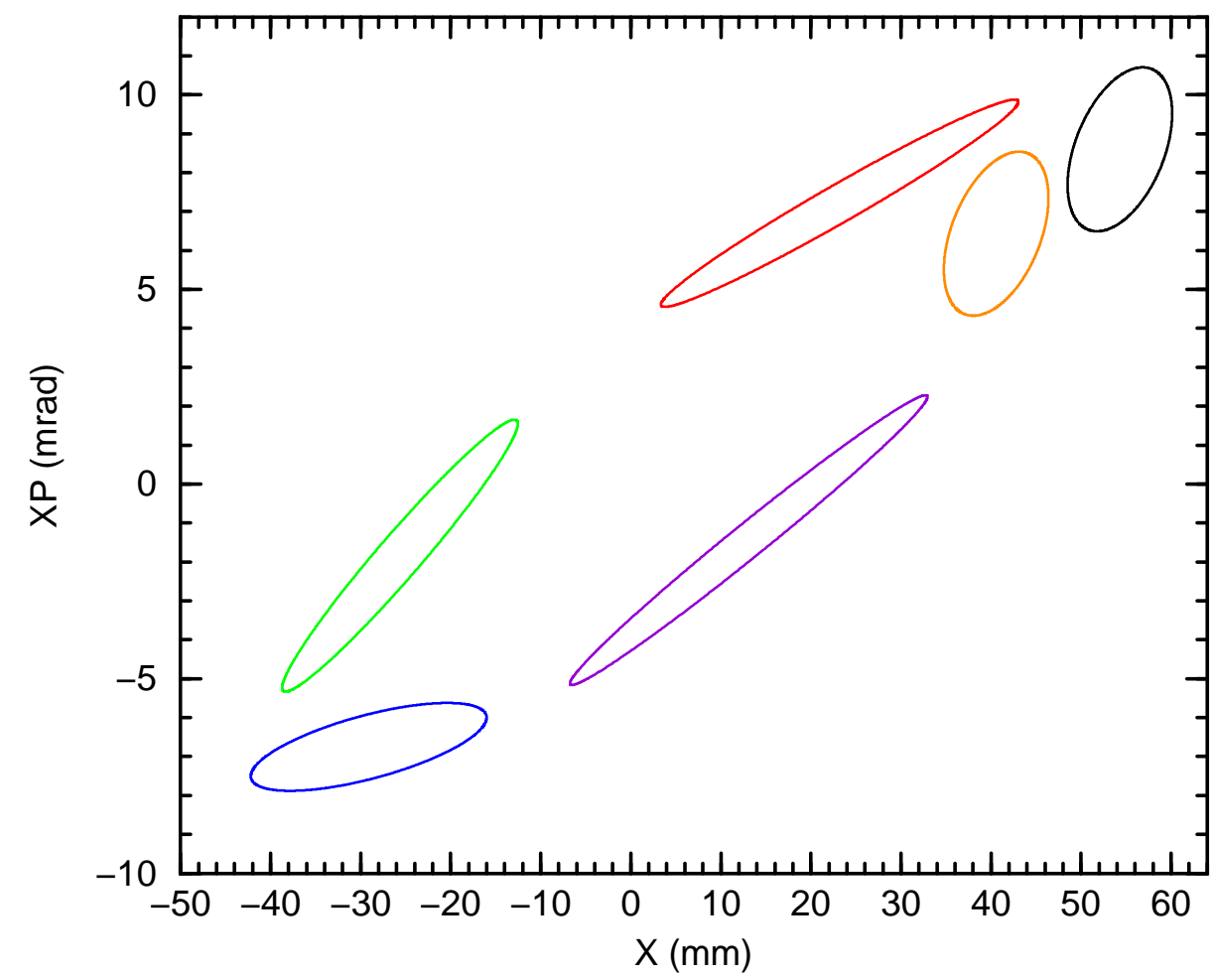

Figure 36: The black, red, green, blue, violet, and orange curves are the beam ellipse after $0,1,2,3,4$, and 5 turns, respectively, from launch time 4.0 turns. The initial (black) ellipse has parameter $B=0.2793$ and area $\pi \epsilon_{0}=11.0 \pi \mathrm{mm}$ milliradians. The outer side of the $1 \mathrm{~mm}$ thick inflector septum is located at $X=48.5 \mathrm{~mm}$. The tunes are $Q_{H}=4.800$ and $Q_{V}=$ 4.810 . 


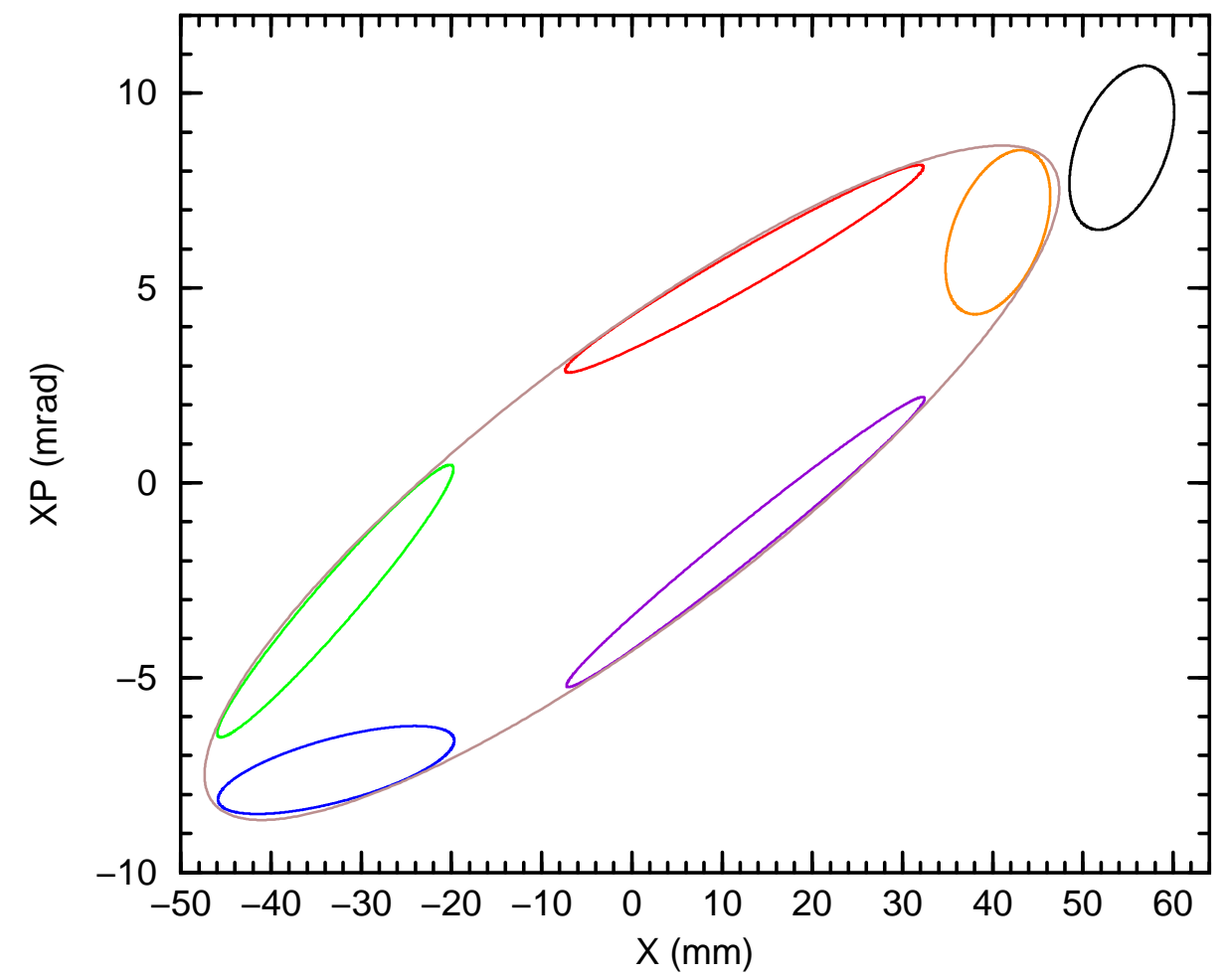

Figure 37: The black, red, green, blue, violet, and orange curves are the beam ellipse after $0,6,7,8,9$, and 10 turns, respectively, from launch time 4.0 turns. The large brown ellipse is the machine acceptance, taken to be $204.5 \pi \mathrm{mm}$ milliradians. The initial (black) ellipse launched at time 4.0 turns therefore ends up inside but very close to the edge of the machine acceptance after the bump has collapsed. 


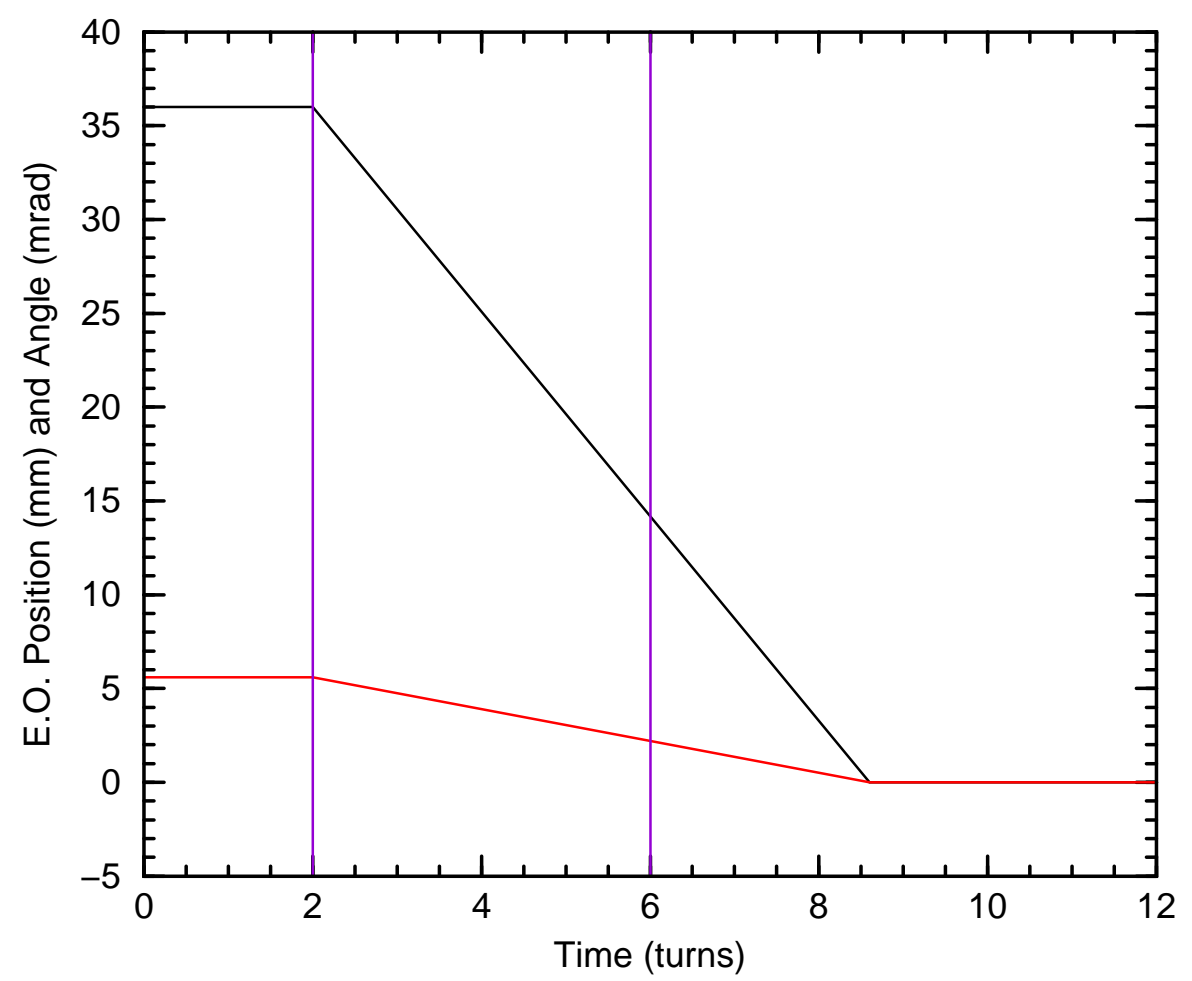

Figure 38: Here the black and red curves are the injection bump position $X_{c}$ and angle $X_{c}^{\prime}=-\alpha_{I} X_{c} / \beta_{I}$ as functions of time for four-turn injection of EBIS beams. The time axis is marked in turns, where one turn is $10.276 \mu \mathrm{s}$. The beam pulse occurs between the two violet lines. The horizontal and vertical tunes are now $Q_{H}=4.70$ and $Q_{V}=4.81$. The MAD program gives $\alpha_{I}=-1.729$ and $\beta_{I}=11.101 \mathrm{~m}$. The magnetic rigidity is taken to be $1.255 \mathrm{Tm}$ which is the expected maximum for EBIS beams. 


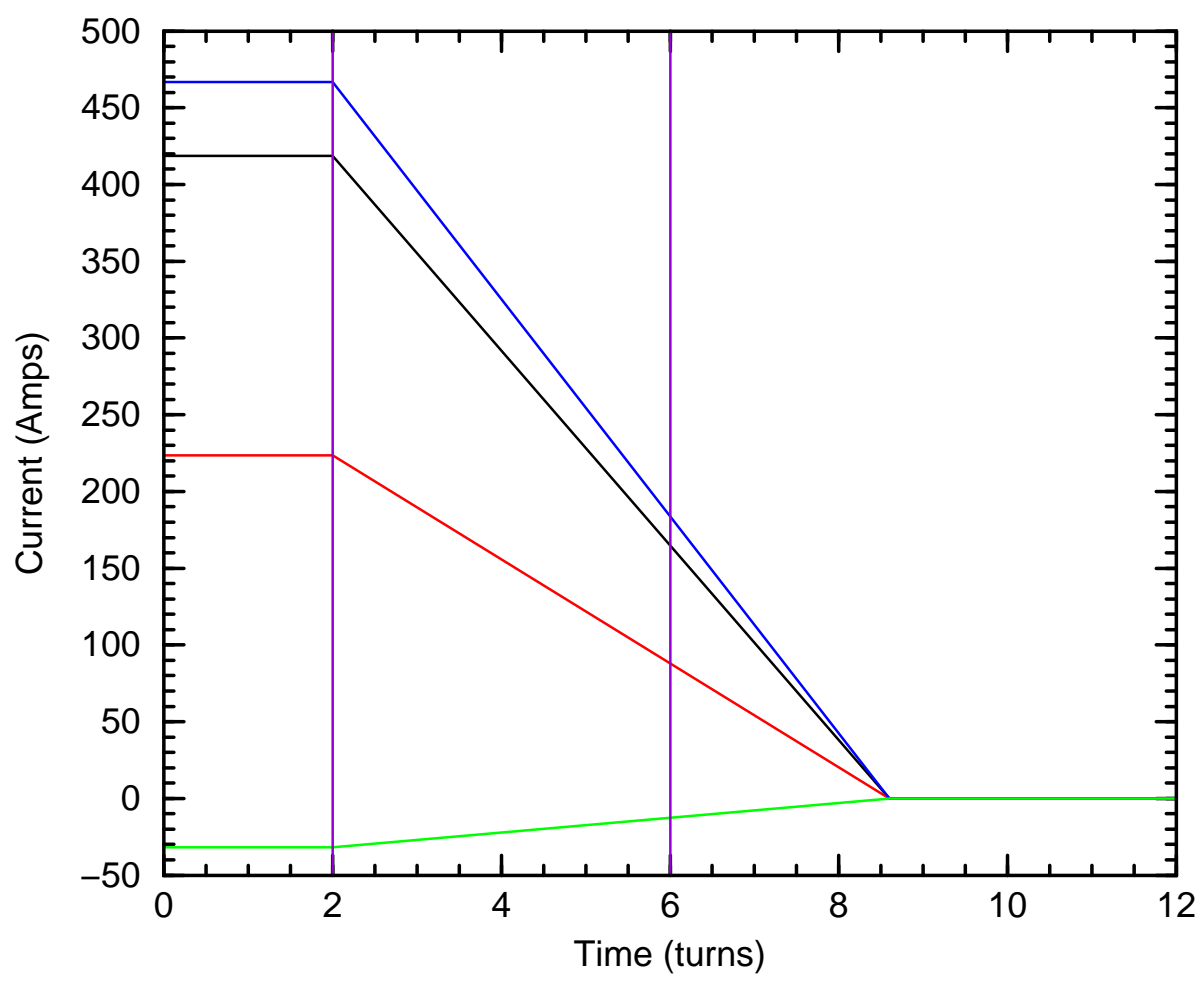

Figure 39: Here the black, red, blue, and green curves are the C1, C3, $\mathrm{C} 7$, and D1 dipole currents, respectively, for the injection bump function of Figure 38. The four-turn EBIS beam pulse occurs between the two violet lines. The rate of fall of the $\mathrm{C} 7$ dipole current (blue curve) is $6.87 \mathrm{~A} / \mu \mathrm{s}$. 


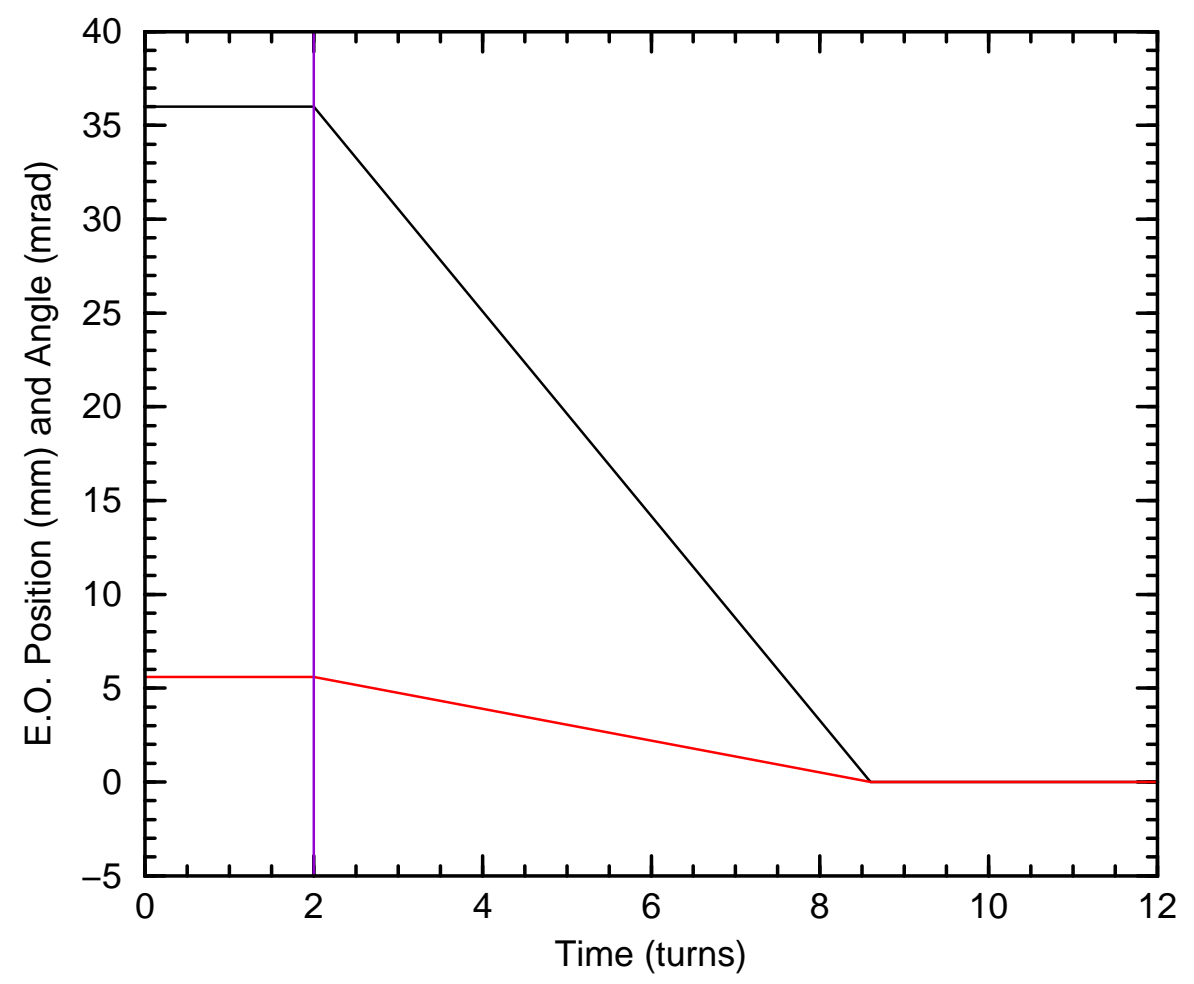

Figure 40: We consider the evolution of a beam ellipse launched from the injection point (i.e. the exit of the $\mathrm{C} 3$ inflector) at time 2.0 turns as indicated by the violet line above. Figures 41 through 43 show the evolution of the beam ellipse. The necessary computations were carried out with Fortran program "EBinject" using MAD Twiss file TwissQH47QV481. 


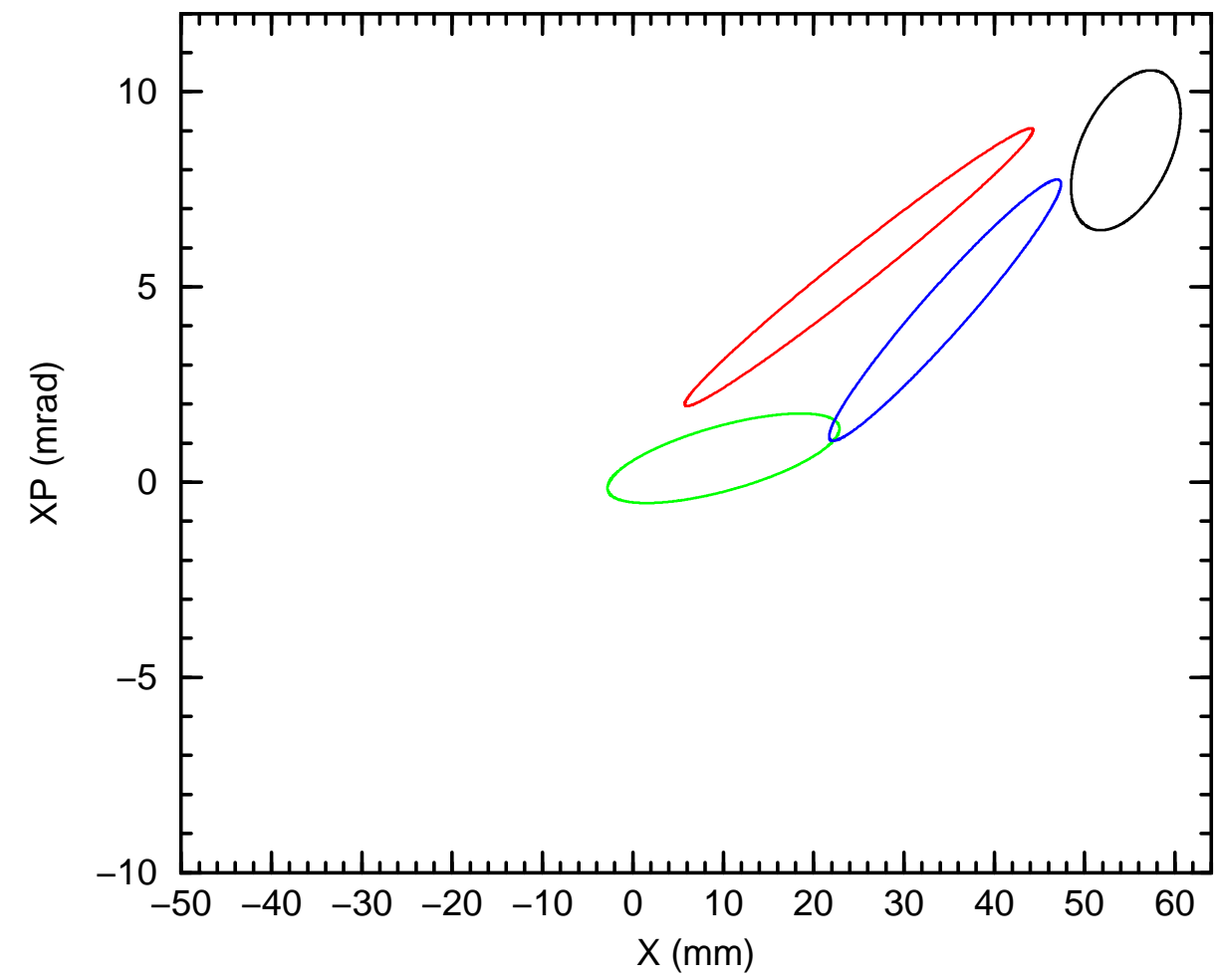

Figure 41: The black, red, green, and blue curves are the beam ellipse after $0,1,2$, and 3 turns, respectively, from launch time 2.0 turns. The initial (black) ellipse has parameter $B=0.3$ and area $\pi \epsilon_{0}=11.0 \pi \mathrm{mm}$ milliradians. The outer side of the $1 \mathrm{~mm}$ thick inflector septum is located at $X=48.5 \mathrm{~mm}$. The tunes are $Q_{H}=4.70$ and $Q_{V}=4.81$. 


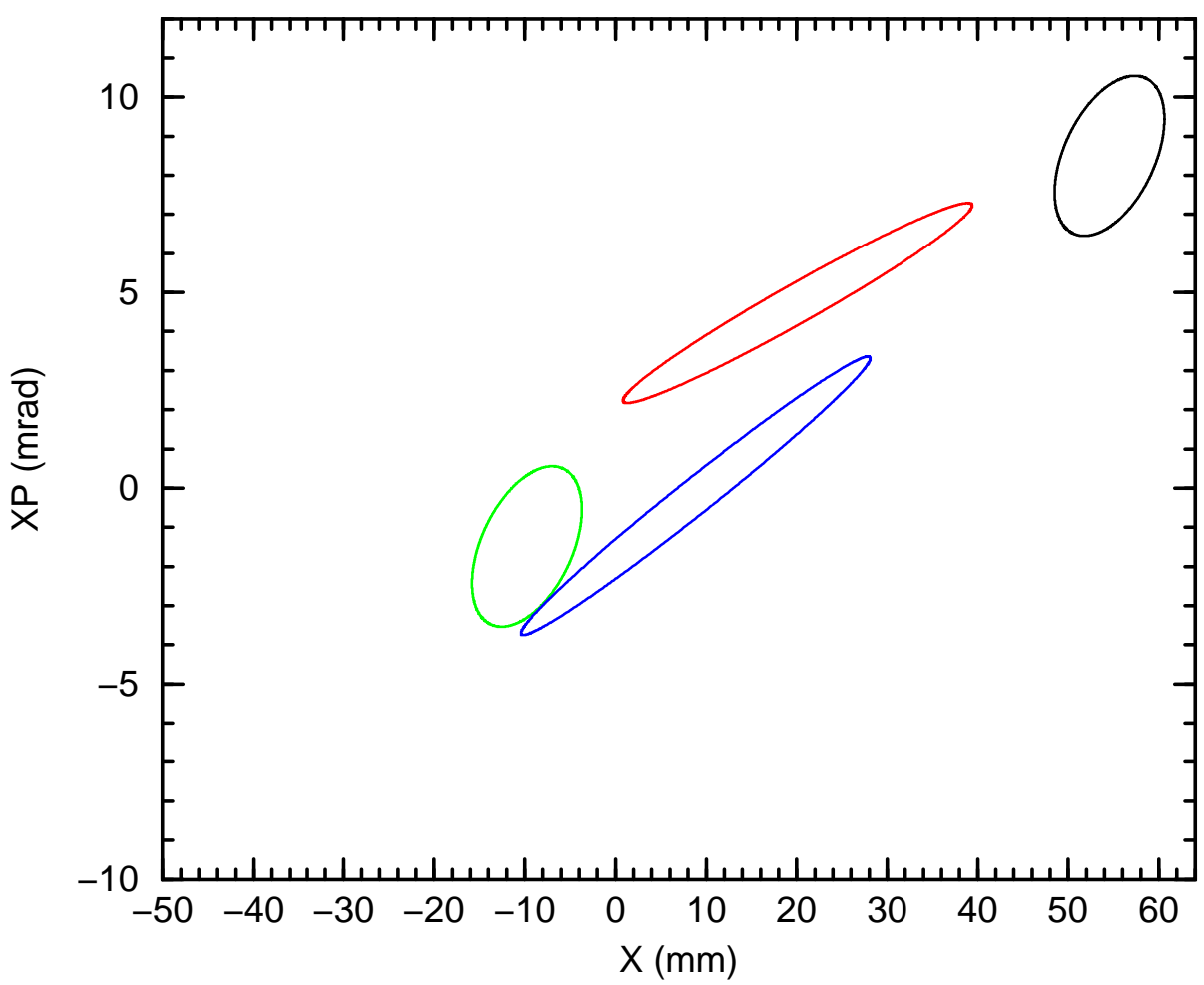

Figure 42: The black, red, green, blue, and violet curves are the beam ellipse after $0,4,5$, and 6 turns, respectively, from launch time 2.0 turns. Note again that the injected ellipse moves with the falling bump. 


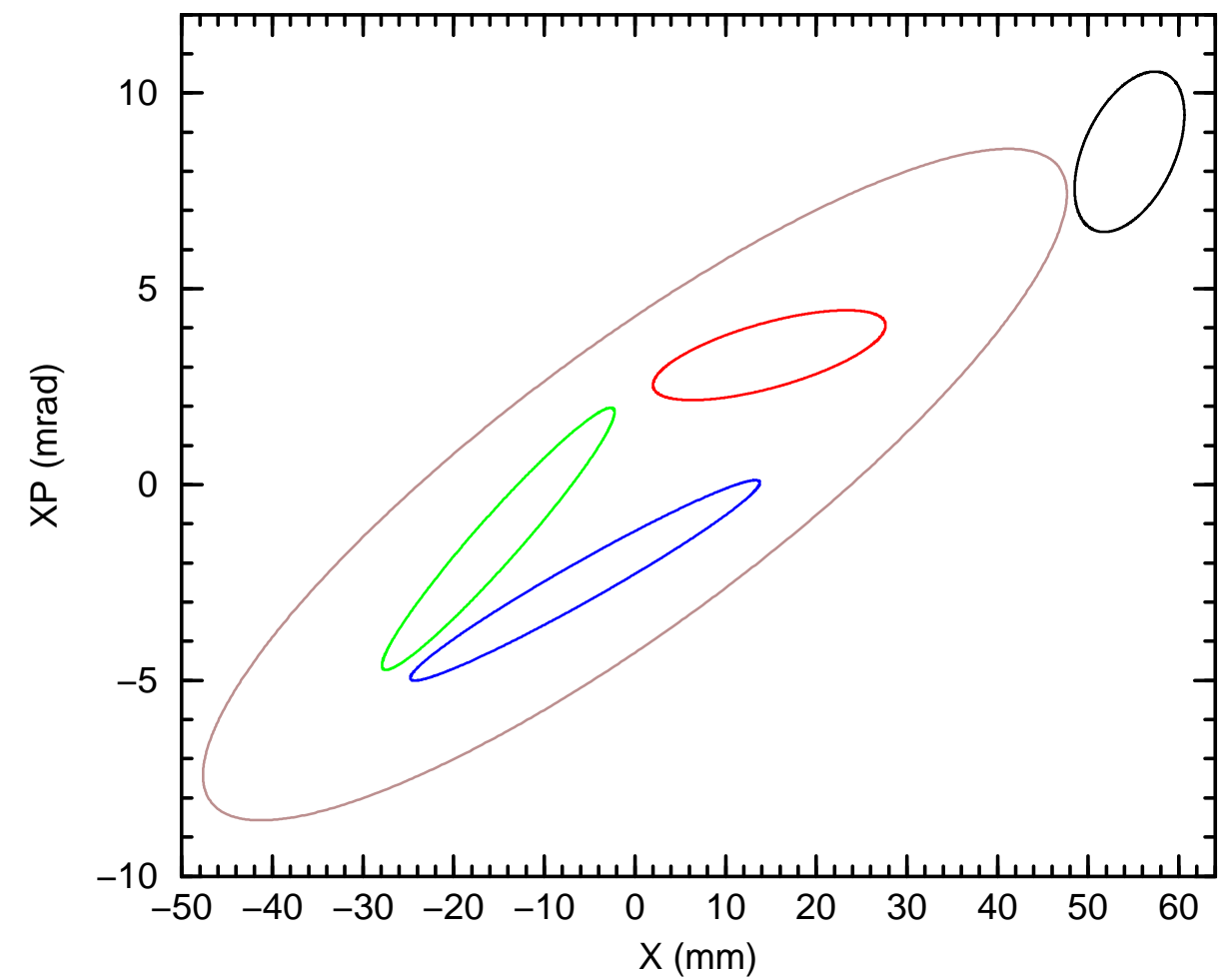

Figure 43: The black, red, green, and blue curves are the beam ellipse after 0 , 7,8 , and 9 turns, respectively, from launch time 2.0 turns. The large brown ellipse is the machine acceptance, taken to be $204.5 \pi \mathrm{mm}$ milliradians. The initial (black) ellipse launched at time 2.0 turns therefore ends up well inside the machine acceptance after the bump has collapsed. 


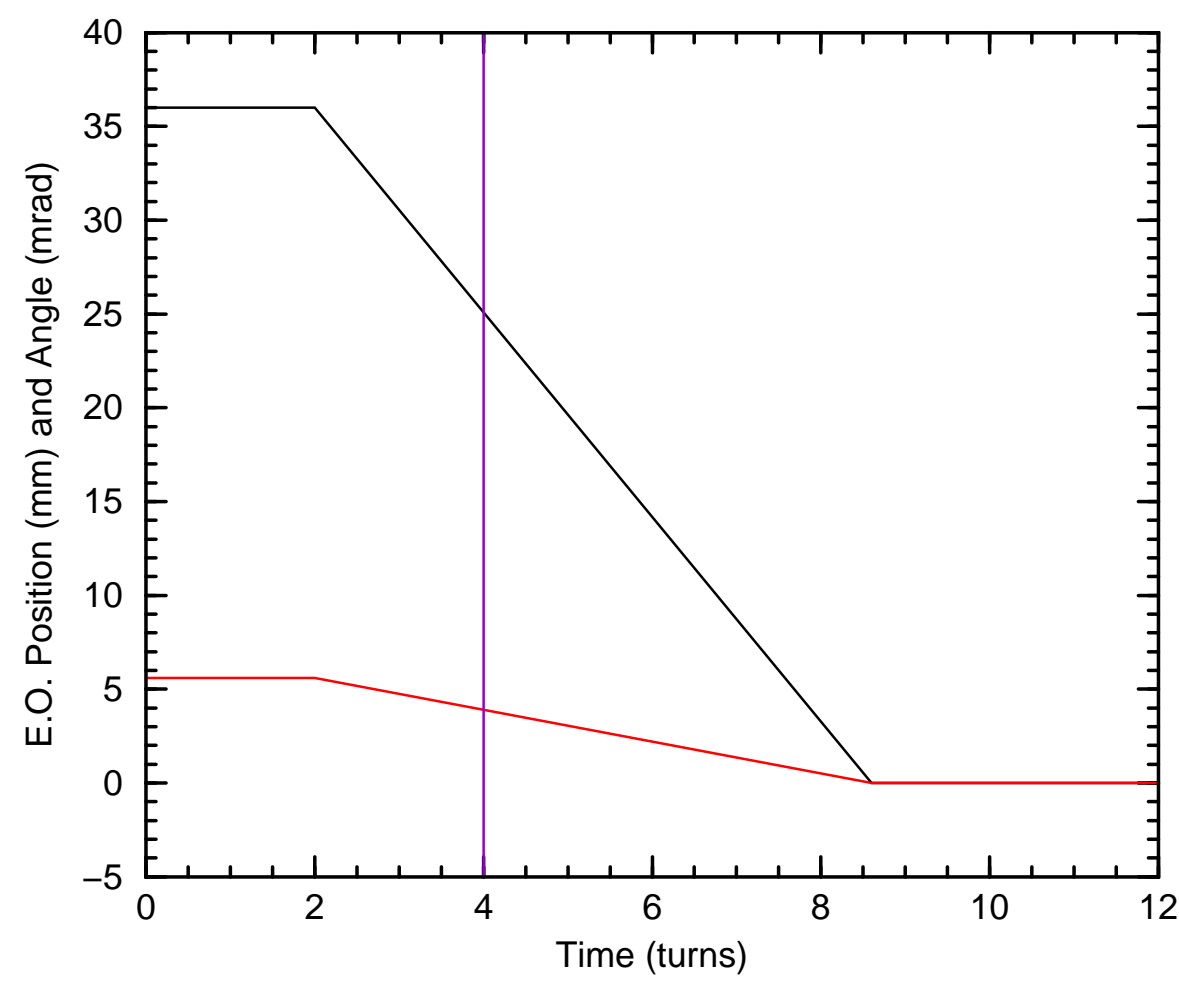

Figure 44: Now consider the evolution of a beam ellipse launched from the injection point at time 4.0 turns as indicated by the violet line above. Figures 45 and 46 show the evolution of the beam ellipse. The necessary computations were carried out with Fortran program "EBinject" using MAD Twiss file TwissQH47QV481. 


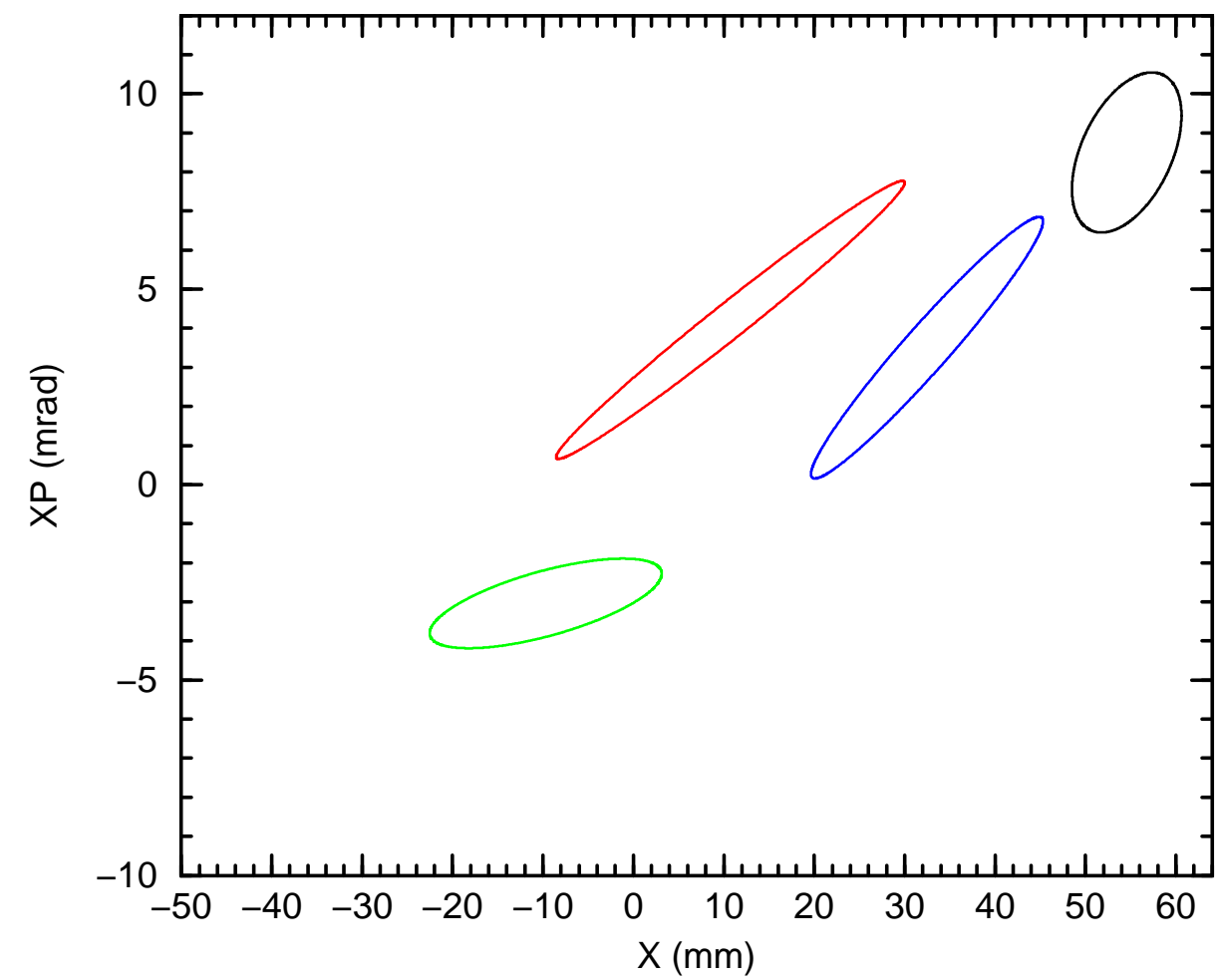

Figure 45: The black, red, green, and blue curves are the beam ellipse after $0,1,2$, and 3 turns, respectively, from launch time 4.0 turns. As before, the initial (black) ellipse has parameter $B=0.3$ and area $\pi \epsilon_{0}=11.0 \pi \mathrm{mm}$ milliradians. The outer side of the $1 \mathrm{~mm}$ thick inflector septum is located at $X=48.5 \mathrm{~mm}$. The tunes are $Q_{H}=4.70$ and $Q_{V}=4.81$. 


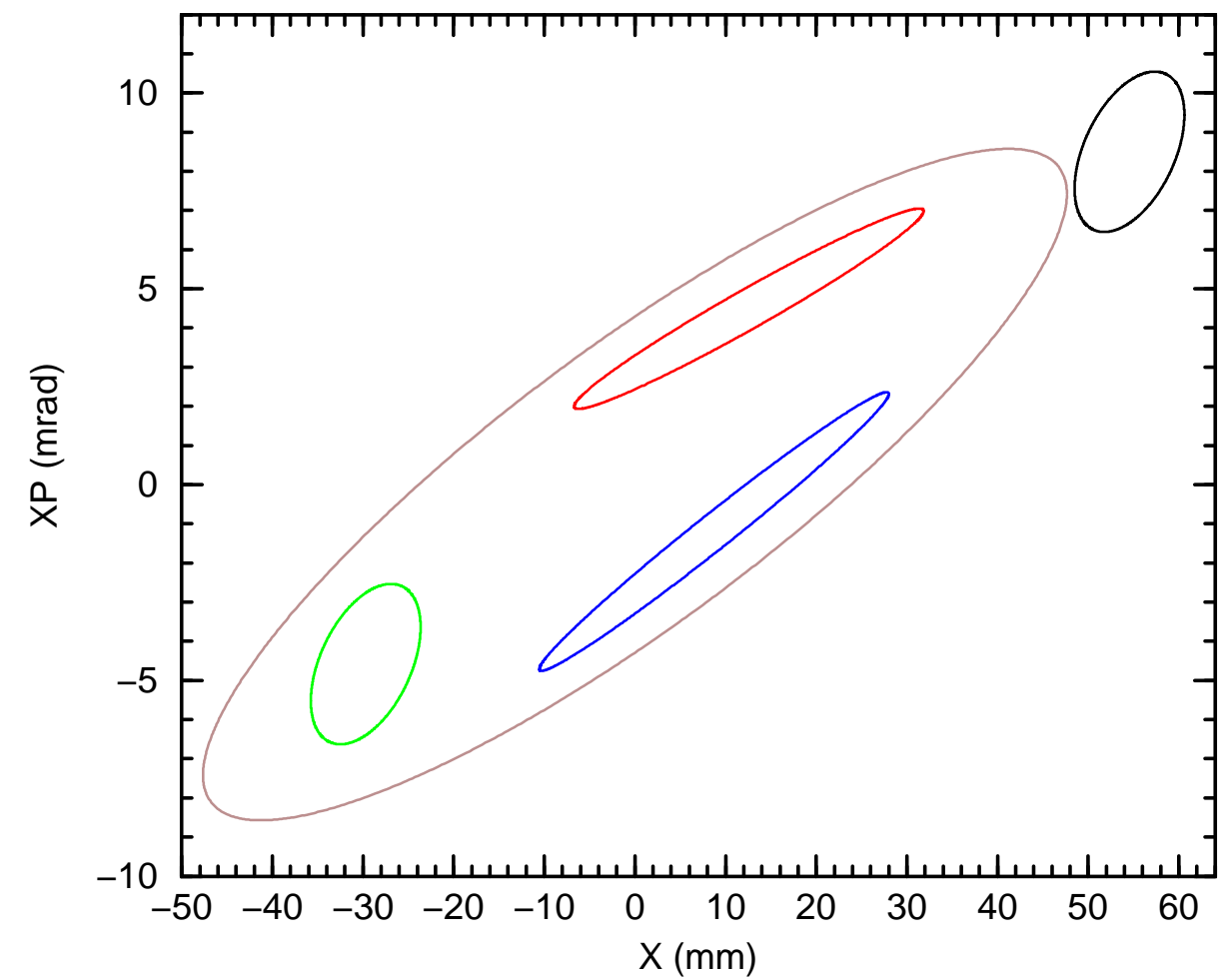

Figure 46: The black, red, green, and blue curves are the beam ellipse after 0 , 4, 5, and 6 turns, respectively, from launch time 4.0 turns. The large brown ellipse is the machine acceptance, taken to be $204.5 \pi \mathrm{mm}$ milliradians. The initial (black) ellipse launched at time 4.0 turns therefore ends up well inside the machine acceptance after the bump has collapsed. 


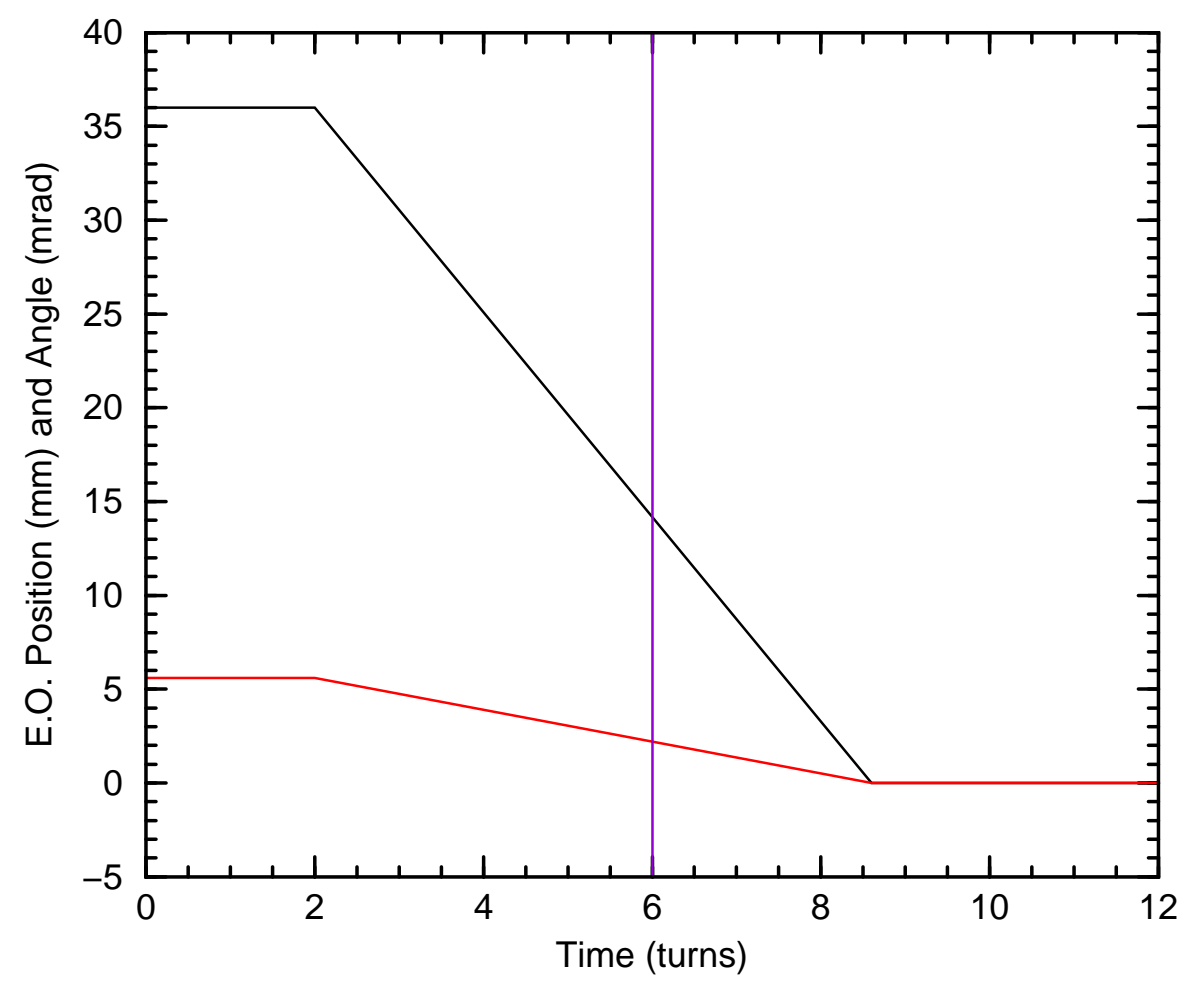

Figure 47: Now consider the evolution of a beam ellipse launched from the injection point at time 6.0 turns as indicated by the violet line above. Figures 48 and 49 show the evolution of the beam ellipse. The necessary computations were carried out with Fortran program "EBinject" using MAD Twiss file TwissQH47QV481. 


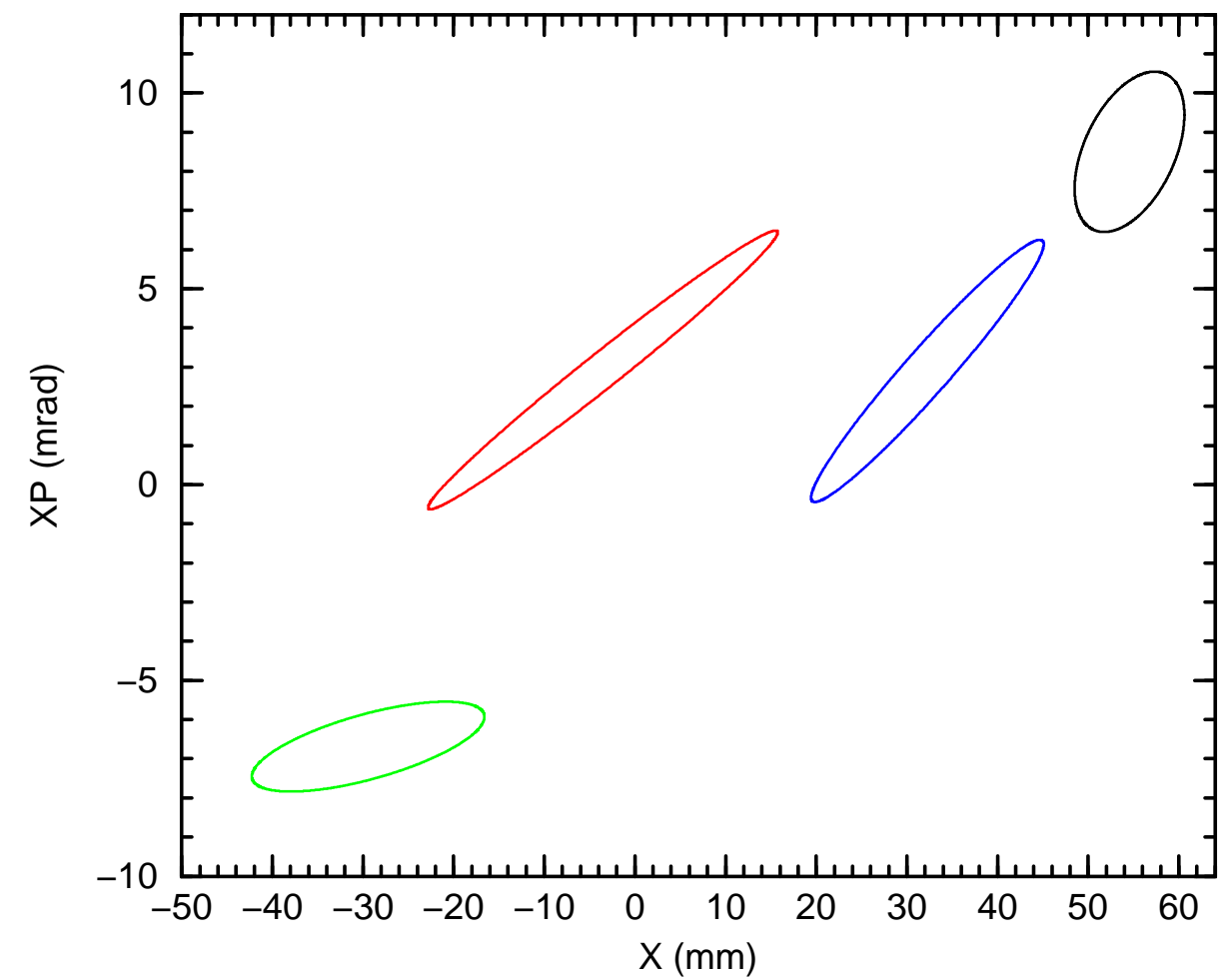

Figure 48: The black, red, green, and blue curves are the beam ellipse after $0,1,2$, and 3 turns, respectively, from launch time 6.0 turns. As before, the initial (black) ellipse has parameter $B=0.3$ and area $\pi \epsilon_{0}=11.0 \pi \mathrm{mm}$ milliradians. The outer side of the $1 \mathrm{~mm}$ thick inflector septum is located at $X=48.5 \mathrm{~mm}$. The tunes are $Q_{H}=4.70$ and $Q_{V}=4.81$. 


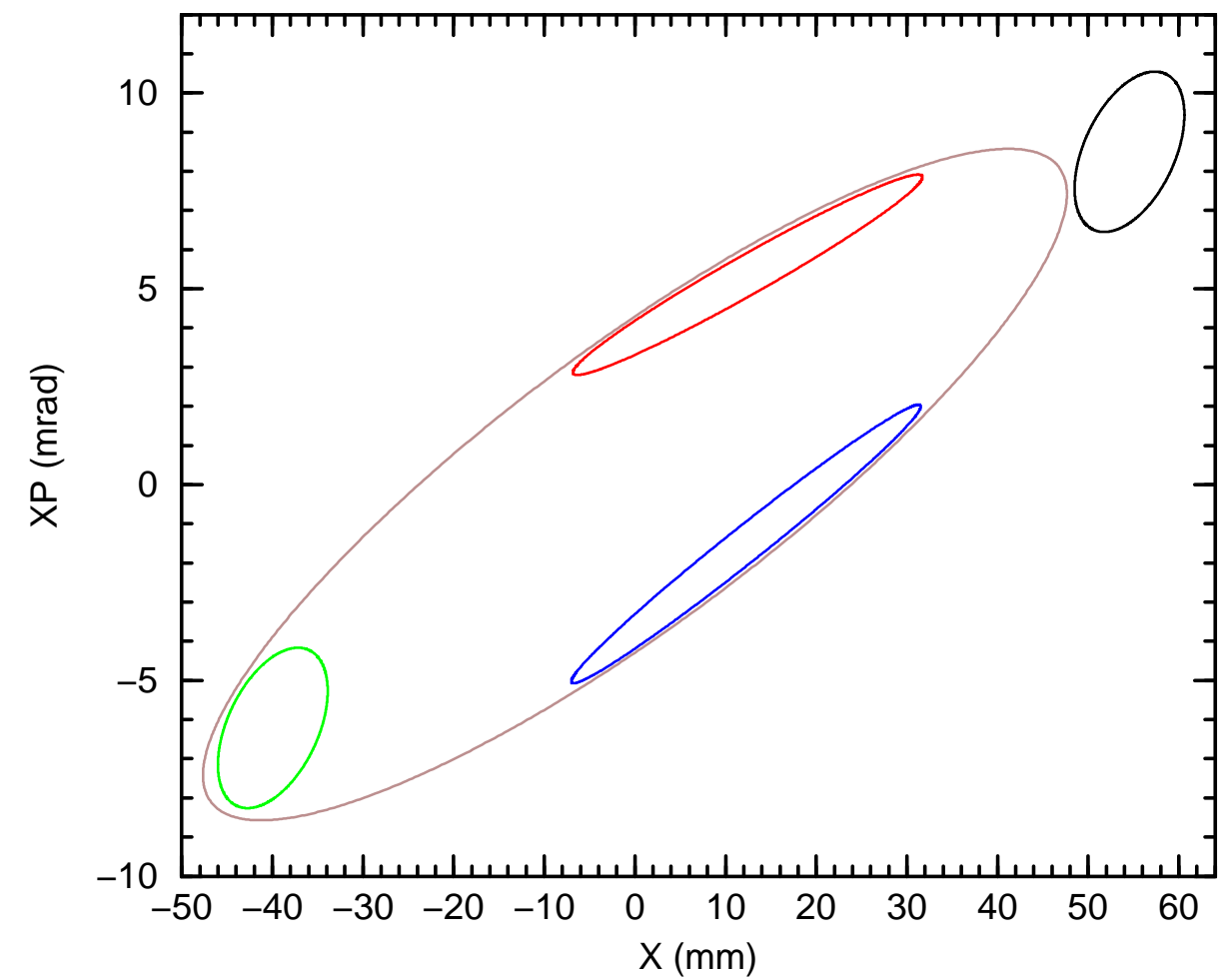

Figure 49: The black, red, green, and blue curves are the beam ellipse after 0 , 4, 5, and 6 turns, respectively, from launch time 6.0 turns. The large brown ellipse is the machine acceptance, taken to be $204.5 \pi \mathrm{mm}$ milliradians. The initial (black) ellipse launched at time 6.0 turns therefore ends up inside but close to the edge of the machine acceptance after the bump has collapsed. 